\title{
Dynamical Mean-Field Theory of Electronic Correlations in Models and Materials
}

Cite as: AIP Conference Proceedings 1297, 339 (2010); https://

doi.org/10.1063/1.3518901

Published Online: 15 November 2010

\section{Dieter Vollhardt}

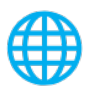

\section{ARTICLES YOU MAY BE INTERESTED IN}

Strongly Correlated Materials: Insights From Dynamical Mean-Field Theory Physics Today 57, 53 (2004); https://doi.org/10.1063/1.1712502

Computational Studies of Quantum Spin Systems

AIP Conference Proceedings 1297, 135 (2010); https://

doi.org/10.1063/1.3518900

Strongly Correlated Electron Materials: Dynamical Mean-Field Theory and Electronic Structure

AIP Conference Proceedings 715, 3 (2004); https://doi.org/10.1063/1.1800733

AlP Author Services

English Language Editing

High-quality assistance from subject specialists

LEARN MORE

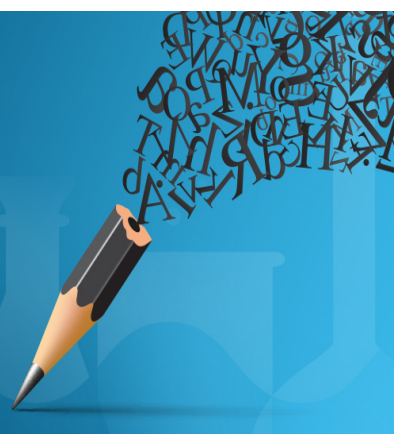




\title{
Dynamical Mean-Field Theory of Electronic Correlations in Models and Materials
}

\author{
Dieter Vollhardt \\ Theoretical Physics III, Center for Electronic Correlations and Magnetism, Institute of Physics, \\ University of Augsburg, 86135 Augsburg, Germany
}

\begin{abstract}
The concept of electronic correlations plays an important role in modern condensed matter physics. It refers to interaction effects which cannot be explained within a static meanfield picture as provided by Hartree-Fock theory. Electronic correlations can have a very strong influence on the properties of materials. For example, they may turn a metal into an insulator (Mott-Hubbard metal-insulator transition). In these lecture notes I (i) introduce basic notions of the physics of correlated electronic systems, (ii) discuss the construction of mean-field theories by taking the limit of high lattice dimensions, (iii) explain the simplifications of the many-body perturbation theory in this limit which provide the basis for the formulation of a comprehensive mean-field theory for correlated fermions, the dynamical mean-field theory (DMFT), (v) derive the DMFT self-consistency equations, and (vi) apply the DMFT to investigate electronic correlations in models and materials.
\end{abstract}

Keywords: Correlated electrons, dynamical mean-field theory, Mott-Hubbard metal-insulator transition, transition-metal oxides, spectroscopy, LDA+DMFT

PACS: 71.10.-w, 71.10.Fd, 71.15.-m, 71.23.-k, 71.27.+a, 71.30.+h, 79.60.-i

\section{CONTENTS}

1 Introduction $\quad 340$

1.1 What are electronic correlations and where do they show up? . . . . . . 340

1.2 Electrons $v s$. Landau quasiparticles . . . . . . . . . . . . . . . . . . . . . 342

1.3 The simplest model for correlated electrons . . . . . . . . . . . . . . 342

2 Mean-field theories for many-body systems 343

2.1 Construction of mean-field theories . . . . . . . . . . . . . . . 343

2.1.1 Motivation for using the limit of high dimensions to construct

mean-field theories . . . . . . . . . . . . . . . . . . . . 344

2.2 A prototypical example: The Weiss mean-field theory for the Ising model 345

2.3 Hartree approximation for the Hubbard model . . . . . . . . . . . . . . 346

3 Lattice fermions in the limit of high dimensions 347

3.1 Scaling of the hopping amplitude . . . . . . . . . . . . . . . 347

3.2 Simplifications of the many-body perturbation theory . . . . . . . . . . 350

3.3 Interactions beyond the on-site interaction . . . . . . . . . . . . . 354

3.3.1 The Hartree approximation revisited . . . . . . . . . . . . . . . 354

3.4 One-particle and two-particle propagators . . . . . . . . . . . . 355

3.5 Weak-coupling correlation energy for the Hubbard Model . . . . . . . . 357

CP1297, Lectures on the Physics of Strongly Correlated Systems XIV: Fourteenth Training Course edited by A. Avella and F. Mancini

(C) 2010 American Institute of Physics 978-0-7354-0851-7/10/\$30.00 
3.6 Consequences of the $k$-independence of the self-energy . . . . . . . 358

4 Dynamical mean-field theory for correlated lattice fermions 359

4.1 Construction of the DMFT by generalizing the coherent potential approximation .................... . . 360

4.1.1 CPA and the limit $d \rightarrow \infty \ldots \ldots$. . . . . . . . . . . . . . . . . . . . . . . . . .

4.1.2 Alternative derivation of CPA . . . . . . . . . . . . 363

4.1.3 Generalization of the CPA approach to interacting systems . . . 366

4.1.4 Exact free energy functional for the Hubbard model in $d=\infty$ and the self-consistency equations . . . . . . . . . 367

4.2 Construction of the DMFT as a self-consistent single-impurity Anderson model ...................... . . 370

4.3 Solution of the DMFT self-consistency equations . . . . . . . . . . 374

5 The Mott-Hubbard Metal-Insulator Transition 375

5.1 DMFT and the three-peak structure of the spectral function . . . . . . 376

6 Electronic correlations and disorder $\quad 380$

6.1 Arithmetic $v s$. geometric averaging over the disorder . . . . . . . . . . 381

6.2 The Anderson-Hubbard model . . . . . . . . . . . . . . . . . 382

7 Theory of electronic correlations in materials $\quad 384$

7.1 The LDA+DMFT approach . . . . . . . . . . . . . . . . . . . . 384

7.2 Single-Particle Spectrum of Correlated Electrons in Materials . . . . . . 386

7.2.1 Excursion: Detection of electronic correlations in solids by photoemission spectroscopy . . . . . . . . . . . . 386

7.2.2 $\mathrm{Sr}_{x} \mathrm{Ca}_{1-x} \mathrm{VO}_{3} \ldots \ldots \ldots \ldots \ldots . \ldots \ldots 38$

$7.2 .3 \mathrm{NiO} \ldots \ldots \ldots . \ldots . \ldots . \ldots 389$

7.3 Correlation induced structural transformations . . . . . . . . . . . 392

8 Kinks in the dispersion of strongly correlated electron systems 394

9 Summary and Outlook 396

\section{INTRODUCTION}

\subsection{What are electronic correlations and where do they show up?}

The term correlations (which means "with relation", from Latin con + relatio) is used not only in physics, but also in many other fields. For example, in grammar the two words either ... or are called a "correlate". Indeed, in a grammatically correct sentence the word either always has to be followed by the word or. Obviously the two words are correlated. In mathematics and natural sciences the term correlation is used to express the fact that the average or expectation value of a product of quantities is usually not 
equal to the product of the averages of the individual quantities:

$$
\langle A B\rangle \neq\langle A\rangle\langle B\rangle .
$$

For example, the mass or charge density $n(\boldsymbol{r})$ of a many-body system at position $\boldsymbol{r}$ is influenced, in general, by the density of the particles at other positions $\boldsymbol{r}^{\prime}$. Therefore the density-density correlation function is not simply given by the product of the average density $n$ :

$$
\left\langle n(\boldsymbol{r}) n\left(\boldsymbol{r}^{\prime}\right)\right\rangle \neq\langle n(\boldsymbol{r})\rangle\left\langle n\left(\boldsymbol{r}^{\prime}\right)\right\rangle=n^{2} .
$$

Indeed, this very property defines correlations: they express effects which go beyond approximations obtained by the factorization of correlation functions, i.e., beyond static mean-field theories such as the Weiss mean-field theory for the Ising model or the Hartree approximation for the Hubbard model.

Correlations in space and time are by no means abstract notions, but occur frequently in everyday life. Persons in an elevator or in a car are strongly correlated both in space and time, and it would be quite inadequate to describe the situation of a person in such a case within a factorization approximation where the influence of the other person(s) is described only by a static mean-field, i.e., a structureless cloud.

As in the case of two persons riding together on an elevator, two electrons with different spin direction occupying the same narrow $d$ or $f$ orbital in a real material are also correlated. Here the degree of correlation can be estimated in a very simplified picture as follows. Assuming the correlated electrons (or rather the quasiparticles, i.e., excitations) to have a well-defined dispersion $\varepsilon_{k}$, their velocity is given by $v_{k}=\frac{1}{\hbar}\left|\nabla_{k} \varepsilon_{k}\right|$. The typical velocity is given by $v_{k} \sim \frac{a}{\tau}$, where $a$ is the lattice spacing and $\tau$ is the average time spent on an atom. The derivative can be estimated as $\frac{1}{\hbar}\left|\nabla_{k} \varepsilon_{k}\right| \sim \frac{1}{\hbar} a W$ since $\left|\nabla_{k}\right| \sim 1 / k \sim a$ and $\left|\varepsilon_{k}\right|$ corresponds to the band overlap $t$ and hence to the band width $W$. Altogether this means that

$$
\tau \sim \frac{\hbar}{W} .
$$

The narrower an orbital, the longer an electron therefore resides on an atom and thereby feels the presence of other electrons. Hence a narrow band width implies strong electronic correlations.

Indeed this is the case for many elements in the periodic table. Namely, in many materials with partially filled $d$ and $f$ electron shells, such as the transition metals $\mathrm{V}, \mathrm{Fe}$, and $\mathrm{Ni}$ and their oxides, or rare-earth metals such as $\mathrm{Ce}$, electrons occupy narrow orbitals. This spatial confinement enhances the effect of the Coulomb interaction between the electrons, making them "strongly correlated". Correlation effects lead to profound quantitative and qualitative changes of the physical properties of electronic systems as compared to non-interacting particles. In particular, they often respond very strongly to changes in external parameters. This is expressed by large renormalizations of the response functions of the system, e.g., of the spin susceptibility and the charge compressibility. Electronic correlations also play an essential role in high temperature superconductivity. In particular, the interplay between the spin, charge and orbital degrees of freedom of the correlated $d$ and $f$ electrons and with the lattice degrees of freedom leads to a wealth of unusual phenomena at low temperatures [1]. These properties cannot be 
explained within conventional mean-field theories, e.g., Hartree-Fock theory, since they describe the interaction only in an average way.

\subsection{Electrons vs. Landau quasiparticles}

Electrons are fermions which obey the Fermi-Dirac statistics. The Pauli exclusion principle implies the existence of a Fermi body of occupied states and thereby of a Fermi surface which distinguishes between occupied states (inside the Fermi body) and empty states (outside the Fermi body). The existence of a Fermi surface is something quite extraordinary. It allows for the formulation of Landau Fermi-liquid theory and thereby for a deep understanding of interacting fermionic systems [2].

In the Landau Fermi-liquid theory a one-to-one correspondence between $\boldsymbol{k}$-states of the non-interacting and the interacting system is assumed. Therefore there exist welldefined $\boldsymbol{k}$-states, called quasiparticles, which have a finite lifetime (the closer they are to the Fermi surface the more well-defined they are, i.e., the longer they live), an effective mass $m^{*}$ and an effective interaction. Quasiparticles are the elementary excitations of a Fermi liquid and determine the entire low temperature thermodynamics of a Landau Fermi liquid. They are rather abstract objects which should not be confused with the particles of the non-interacting system. The Fermi-liquid concept is independent of the strength of the bare interaction between particles. Hence it can not only describe simple metals such as potassium where $m^{*}$ is not much different from the bare electronic mass, but even "heavy fermion systems" such as $\mathrm{UBe}_{13}$ where $m^{*}$ can be a factor 1000 larger than the bare electronic mass [3].

\subsection{The simplest model for correlated electrons}

The simplest model describing interacting electrons in a solid is the one-band, spin1/2 Hubbard model [4-6] where the interaction between the electrons is assumed to be so strongly screened that it is taken as purely local. The Hamiltonian consists of two terms, the kinetic energy $\hat{H}_{0}$ and the interaction energy $\hat{H}_{I}$ (here and in the following operators are denoted by a hat):

$$
\begin{aligned}
\hat{H} & =\hat{H}_{0}+\hat{H}_{I} \\
\hat{H}_{0} & =\sum_{\left\langle\boldsymbol{R}_{i}, \boldsymbol{R}_{j}\right\rangle} \sum_{\sigma} t_{i j} \hat{c}_{i \sigma}^{+} \hat{c}_{j \sigma}=\sum_{\boldsymbol{k}, \sigma} \varepsilon_{\boldsymbol{k}} \hat{n}_{\boldsymbol{k} \sigma} \\
\hat{H}_{\mathrm{I}} & =U \sum_{\boldsymbol{R}_{i}} \hat{n}_{i \uparrow} \hat{n}_{i \downarrow},
\end{aligned}
$$

where $\hat{c}_{i \sigma}^{+}\left(\hat{c}_{i \sigma}\right)$ are creation (annihilation) operators of electrons with spin $\sigma$ at site $\boldsymbol{R}_{i}$, and $\hat{n}_{i \sigma}=\hat{c}_{i \sigma}^{+} \hat{c}_{i \sigma}$. The Fourier transform of the kinetic energy in (4b), where $t_{i j}$ is the hopping amplitude, involves the dispersion $\varepsilon_{k}$ and the momentum distribution operator $\hat{n}_{k \sigma}$. 


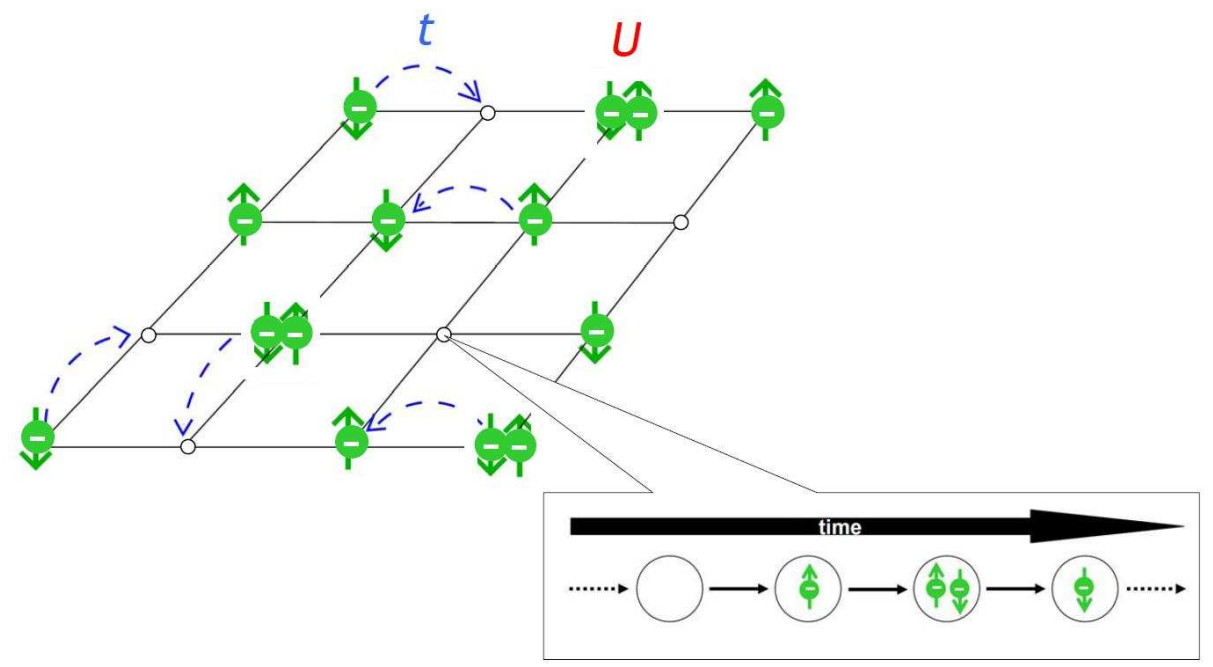

FIGURE 1. Schematic illustration of interacting electrons in a solid in terms of the Hubbard model. The ions appear only as a rigid lattice (here represented as a square lattice). The electrons, which have a mass, a negative charge, and a spin ( $\uparrow$ or $\downarrow$ ), move from one lattice site to the next with a hopping amplitude $t$. The quantum dynamics thus leads to fluctuations in the occupation of the lattice sites as indicated by the time sequence. When two electrons meet on a lattice site (which is only possible if they have opposite spin because of the Pauli exclusion principle) they encounter an interaction $U$. A lattice site can either be unoccupied, singly occupied ( $\uparrow$ or $\downarrow$ ), or doubly occupied.

A schematic picture of the Hubbard model is shown in Fig. 1. When we look only at a single site of this lattice model, this site will sometimes be empty, singly occupied or doubly occupied. In particular, for strong repulsion $U$ double occupations are energetically very unfavorable and are therefore strongly suppressed. In this situation $\left\langle\hat{n}_{i \uparrow} \hat{n}_{i \downarrow}\right\rangle$ must not be factorized since $\left\langle\hat{n}_{i \uparrow} \hat{n}_{i \downarrow}\right\rangle \neq\left\langle\hat{n}_{i \uparrow}\right\rangle\left\langle\hat{n}_{i \downarrow}\right\rangle$. Otherwise, correlation phenomena such as the Mott-Hubbard metal-insulator transition are eliminated from the very beginning. This explains why Hartree-Fock-type mean-field theories are generally insufficient to explain the physics of electrons in the paramagnetic phase for strong interactions.

The Hubbard model looks very simple. However, the competition between the kinetic energy and the interaction leads to a complicated many-body problem. which is impossible to solve analytically, except in dimension $d=1$ [7]. This model provides the basis for most of the theoretical research on correlated electrons during the last decades.

\section{MEAN-FIELD THEORIES FOR MANY-BODY SYSTEMS}

\subsection{Construction of mean-field theories}

It is well known that theoretical investigations of quantum-mechanical many-body systems are faced with severe technical problems, particularly in those dimensions 
which are most interesting to us, i. e., $d=2,3$. This is due to the complicated dynamics and, in the case of fermions, the non-trivial algebra introduced by the Pauli exclusion principle. In the absence of exact methods there is clearly a great need for reliable, controlled approximation schemes. Their construction is not straightforward.

In the statistical theory of classical and quantum-mechanical systems a rough, overall description of the properties of a model is often obtained within a so-called mean-field theory. Although the term is frequently used, the actual meaning of what a mean-field theory is or should be is rather vague, because there is no unique prescription of how to construct such a theory (and sometimes it is only a respectable name for a questionable approximation ... ). Hence every time one encounters the term "mean-field theory", one should ask about the reliability of this approximation, i. e., about its range of validity with respect to the input parameters and its thermodynamic consistency.

There exists a well-established branch of approximation techniques which makes use of the simplifications that occur when some parameter is taken to be large (in fact, infinite), e.g., the length of the spins $S$, the spin degeneracy $N$, the coordination number $Z$ (the number of nearest neighbors of a lattice site). Investigations in this limit, supplemented, if at all possible, by an expansion in the inverse of the large parameter, often provide valuable insight into the fundamental properties of a system even when this parameter is not large.

One of the best-known mean-field theories is the Weiss molecular-field theory for the Ising model [8]. It is a prototypical single-site mean-field theory which becomes exact for infinite-range interaction, as well as in the limit of the coordination number $Z \rightarrow \infty$ or $^{1}$ the dimension $d \rightarrow \infty$. In the latter case $1 / Z$ or $1 / d$ is a small parameter which can sometimes be used to improve the mean-field theory systematically. This meanfield theory contains no unphysical singularities and is applicable for all values of the input parameters, i.e., coupling parameters, magnetic field, and temperature. It is also diagrammatically controlled [10]. Insofar it is a very respectable approximation which sets very high standards for other mean-field theories.

\subsubsection{Motivation for using the limit of high dimensions to construct mean-field theories}

In a perfectly crystalline system every lattice site has the same number of nearest neighbors $Z$. In three dimensions $(d=3)$ one has $Z=6$ for a simple cubic lattice $(Z=2 d$ for a hypercubic lattice in general dimensions $d$ ), $Z=8$ for a bcc lattice and $Z=12$ for an fcc-lattice. The dimensionality of a lattice system is directly described by the number $Z$

\footnotetext{
${ }^{1}$ For regular lattices, e.g., Bravais-lattices, both a dimension $d$ and a coordination number $Z$ can be defined. In this case either $d$ or $Z$ can be used alternatively as an expansion parameter. However, there exist other lattices (or rather graphs) which cannot be associated with a physical dimension $d$ although a coordination number $Z$ is well-defined. The best-known example is the Bethe lattice, an infinitely extended Cayley tree [8,9], which is not a regular lattice because it does not have loops. The coordination number $Z$ is therefore a very useful parameter for theoretical investigations of lattice models, although the dimension $d$ is the more general physical parameter. In the following discussion we mostly use both $d$ and $Z$ in parallel.
} 
(see footnote 1 ). Since $Z \sim \mathscr{O}(10)$ is already quite large in $d=3$, such that $1 / Z$ is rather small, it is only natural and in the general spirit of theoretical physics to consider the extreme limit $Z \rightarrow \infty$ to simplify the problem. Later, if possible, one can try to improve the result by expanding in the small parameter $1 / Z$. The limit $d \rightarrow \infty$ is not as academic as it might seem. In fact, it turns out that several standard approximation schemes which are commonly used to explain experimental results in dimension $d=3$, are exact only in $d=\infty[11]$.

\subsection{A prototypical example: The Weiss mean-field theory for the Ising model}

In the case of classical spin models (e.g., Ising, Heisenberg) the $Z \rightarrow \infty$ limit is wellknown $[8,10]$. It leads to the results of the Weiss molecular-field theory which may be viewed as the prototypical method for constructing a mean-field theory. The Hamiltonian for the Ising model with nearest-neighbor (NN) coupling is given by

$$
H=-\frac{1}{2} J \sum_{\left\langle\boldsymbol{R}_{i}, \boldsymbol{R}_{j}\right\rangle} S_{i} S_{j},
$$

where we assume ferromagnetic coupling $(J>0)$. Every spin $S_{i}$ interacts with a local field $h_{i}$, produced by its nearest neighbors at site $\boldsymbol{R}_{i}$. In the Weiss mean-field approach the two-spin interaction in (5) is decoupled, i. e., $H$ is replaced by a mean-field Hamiltonian

$$
H^{\mathrm{MF}}=-h_{\mathrm{MF}} \sum_{\boldsymbol{R}_{i}} S_{i}+E_{\text {shift }} \cdot
$$

Now a spin $S_{i}$ interacts only with a global ("molecular") field

$$
\begin{aligned}
h_{\mathrm{MF}} & =J \sum_{\boldsymbol{R}_{j}}^{(i)}\left\langle S_{j}\right\rangle \\
& \equiv J\langle S\rangle .
\end{aligned}
$$

Here \langle\rangle indicates the thermal average, $E_{\text {shift }}=\frac{1}{2} L J Z\langle S\rangle^{2}$ is a constant energy shift with $L$ as the number of lattice sites and the superscript $(i)$ implies summation over only $\mathrm{NN}$-sites of $\boldsymbol{R}_{i}$. This corresponds to the factorization

$$
\left\langle\left[S_{i}-\langle S\rangle\right]\left[S_{j}-\langle S\rangle\right]\right\rangle \equiv 0,
$$

whereby correlated fluctuations of spins at sites $\boldsymbol{R}_{i}$ and $\boldsymbol{R}_{j}$ are neglected. In the limit $Z \rightarrow \infty$ the coupling constant $J$ has to be rescaled as

$$
J \rightarrow \frac{J^{*}}{Z}, J^{*}=\mathrm{const}
$$

for $h_{\mathrm{MF}}$ to remain finite. In this limit the factorization procedure (7), and hence the replacement of (5), by the mean-field Hamiltonian (6a), becomes exact $[12,13]$. 


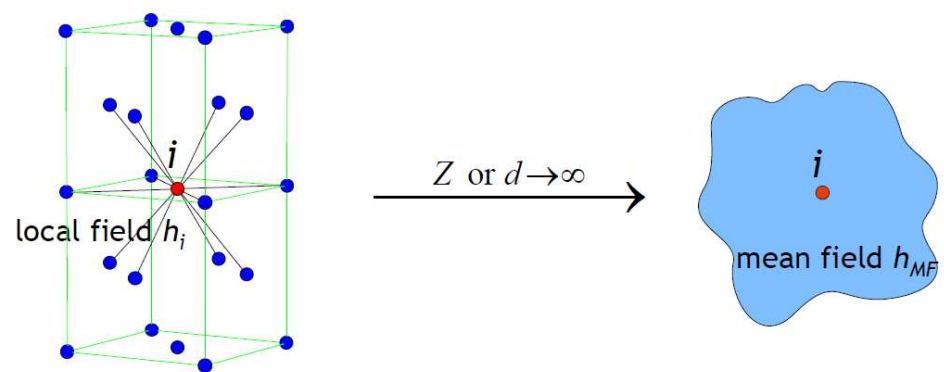

FIGURE 2. Already in three dimensions $(d=3)$ the coordination number $Z$ of a lattice can be quite high, as in the face-centered cubic lattice where $Z=12$. In the limit $Z \rightarrow \infty$, or equivalently $d \rightarrow \infty$, the Ising model effectively reduces to a single-site problem where the local field $h_{i}$ is replaced by a global mean ("molecular") field $h_{\mathrm{MF}}$.

Eq. (6a) implies that in the limit $Z \rightarrow \infty$ fluctuations in the "bath" of surrounding neighbors become unimportant, such that the surrounding of any site is completely described by a single mean-field parameter $h_{\mathrm{MF}}$ (see Fig. 2). Hence the Hamiltonian becomes purely local

$$
\begin{gathered}
H^{\mathrm{MF}}=\sum_{\boldsymbol{R}_{i}} H_{i}+E_{\text {shift }} \\
H_{i}=-h_{\mathrm{MF}} S_{i} .
\end{gathered}
$$

Thereby the problem reduces to an effective single-site problem. The value of $\langle S\rangle$ is determined by the Curie-Weiss (or Bragg-Williams) self-consistent equation

$$
\langle S\rangle=\tanh \left(\beta J^{*}\langle S\rangle\right),
$$

where $\beta=1 / T$ (here $k_{B}=1$ ).

It should be noted that the scaling (8) is typical for localized spin models with isotropic coupling, i. e., when the spatial average $\overline{J_{i j}} \equiv J$ is non-zero. On the other hand, in the classical spin glass problem with random coupling one has $\bar{J}_{i j}=0$, but $\overline{J_{i j}^{2}} \neq 0$. In this case a different kind of scaling, namely $J_{i j} \rightarrow J_{i j}^{*} / \sqrt{Z}$, has to be used [14].

\subsection{Hartree approximation for the Hubbard model}

To elucidate some of the shortcomings of conventional mean-field theories for Hubbard-type models, we consider the Hartree approximation ${ }^{2}$, which corresponds to a factorization of the interaction term in direct analogy to the factorization of the spins in

\footnotetext{
2 The Hubbard interaction acts only between electrons with opposite spin; therefore the Hartree-Fock approximation does not lead to an exchange term.
} 
the Ising model. To this end let us consider a generalization of Hubbard model

$$
\hat{H}=\hat{H}_{\text {kin }}+\frac{1}{2} \sum_{\boldsymbol{R}_{i}, \boldsymbol{R}_{j}} \sum_{\sigma \sigma^{\prime}} V_{i j}^{\sigma \sigma^{\prime}} \hat{n}_{i \sigma} \hat{n}_{j \sigma^{\prime}}
$$

where the interaction $V_{i j}^{\sigma \sigma^{\prime}}$ describes a local part, $U \delta_{\sigma,-\sigma^{\prime}}$, i. e., the Hubbard interaction, as well as a nearest-neighbor contribution $V^{\sigma \sigma^{\prime}}$. The form of the interaction term is similar to (5) for the Ising model. In the spirit of a conventional mean-field approximation the two-particle interaction in (12) is factorized, i. e., $\hat{H}_{\text {ext }}$ is replaced by

$$
\hat{H}^{\mathrm{MF}}=\hat{H}_{\mathrm{kin}}+\sum_{\boldsymbol{R}_{i}, \sigma} \hat{n}_{i \sigma}\left\langle\hat{h}_{i \sigma}\right\rangle+E_{\text {shift }}
$$

in complete analogy with the Weiss molecular field theory for the Ising model in Sec. 2.2. In (13) a $\sigma$-electron at site $\boldsymbol{R}_{i}$ interacts only with a local field (a c-number)

$$
\left\langle\hat{h}_{i \sigma}\right\rangle=\sum_{\boldsymbol{R}_{j}, \sigma}^{(i)} V_{i j}^{\sigma \sigma^{\prime}}\left\langle\hat{n}_{j \sigma}\right\rangle .
$$

The above decoupling of the operators is equivalent to the Hartree approximation which sets

$$
\left\langle\left[\hat{n}_{i \sigma}-\left\langle\hat{n}_{i \sigma}\right\rangle\right]\left[\hat{n}_{j \sigma^{\prime}}-\left\langle\hat{n}_{j \sigma^{\prime}}\right\rangle\right]\right\rangle \equiv 0
$$

for all $i, j, \sigma, \sigma^{\prime}$. Thereby correlated fluctuations on the sites $\boldsymbol{R}_{i}$ and $\boldsymbol{R}_{j}$ are neglected. Although (13) is a one-particle problem it cannot be solved exactly in any systematic way, since, in principle, the potential, i. e., the mean field $\left\langle\hat{h}_{i \sigma}\right\rangle$, may be an arbitrarily complicated function of position.

An obvious question concerning (13) is, whether the mean-field decoupling ever becomes exact for all values of $U, V^{\sigma \sigma^{\prime}}$ and $n$, i. e., beyond the weak-coupling or low-density limit. How about the limit $d \rightarrow \infty$ ? We will come back to this question in Sec. 3.3.1 once we understood how this limit can be employed in the case of lattice fermion models.

\section{LATTICE FERMIONS IN THE LIMIT OF HIGH DIMENSIONS}

\subsection{Scaling of the hopping amplitude}

It is natural to ask whether the limit $d \rightarrow \infty$ may also be useful in the investigation of lattice models with itinerant quantum-mechanical degrees of freedom and, in particular, in the case of the Hubbard model. Following Metzner and Vollhardt [15] we take a look at the kinetic energy term (4b), since the interaction term is purely local and is thereby completely independent of the lattice structure and the dimension. For nearest-neighbor 
hopping on a d-dimensional hypercubic lattice (where $Z=2 d$ ) $\varepsilon_{k}$ is given by ${ }^{3}$

$$
\varepsilon_{k}=-2 t \sum_{i=1}^{d} \cos k_{i}
$$

The density of states (DOS) corresponding to $\varepsilon_{k}$ is

$$
N_{d}(\omega)=\sum_{k} \delta\left(\hbar \omega-\varepsilon_{k}\right)
$$

This is simply the probability density for finding $\omega=\varepsilon_{k}$ for a random choice of $\boldsymbol{k}=$ $\left(k_{1}, \ldots, k_{d}\right)$. If the $k_{i}$ are chosen randomly, $\varepsilon_{\boldsymbol{k}}$ in (16) is the sum of (independent) random numbers $-2 t \cos k_{i}$. The central limit theorem then implies that in the limit $d \rightarrow \infty$ the DOS is given by a Gaussian

$$
N_{d}(\omega) \stackrel{d \rightarrow \infty}{\longrightarrow} \frac{1}{2 t \sqrt{\pi d}} \exp \left[-\left(\frac{\omega}{2 t \sqrt{d}}\right)^{2}\right] .
$$

Unless $t$ is scaled properly with $d$ this DOS will become arbitrarily broad and featureless for $d \rightarrow \infty$. Clearly only the "quantum" scaling

$$
t \rightarrow \frac{t^{*}}{\sqrt{d}}, t^{*}=\text { const. }
$$

yields a non-trivial DOS $[15,16]$ :

$$
N_{\infty}(\omega)=\frac{1}{\sqrt{2 \pi} t^{*}} \exp \left[-\frac{1}{2}\left(\frac{\omega}{t^{*}}\right)^{2}\right] .
$$

This DOS does not have any van Hove singularities. The reason for this can be seen when $N_{d}(\omega)$ is calculated explicitly from $(17)[16,17]$. Expressing the $\delta$-function as a Fourier series one has

$$
\begin{aligned}
N_{d}(\omega) & =\prod_{i=1}^{d} \int_{-\pi}^{\pi} \frac{d k_{i}}{2 \pi} \int_{-\infty}^{\infty} d \tau e^{i \tau\left(\omega-\varepsilon_{k}\right)} \\
& =\int_{-\infty}^{\infty} d \tau e^{i \omega \tau}\left[J_{0}(2 \tau t)\right]^{d},
\end{aligned}
$$

where $J_{0}(x)=1-x^{2}+\mathscr{O}\left(x^{4}\right), x \ll 1$, is the zero-order Bessel-function. For $d \gg 1$ the main contribution to the integral comes from the first extremum of $J_{0}(x)$, i. e., $|\tau| \lesssim$

\footnotetext{
${ }^{3}$ In the following we set Planck's constant $\hbar$, Boltzmann's constant $k_{B}$, and the lattice spacing equal to unity.
} 

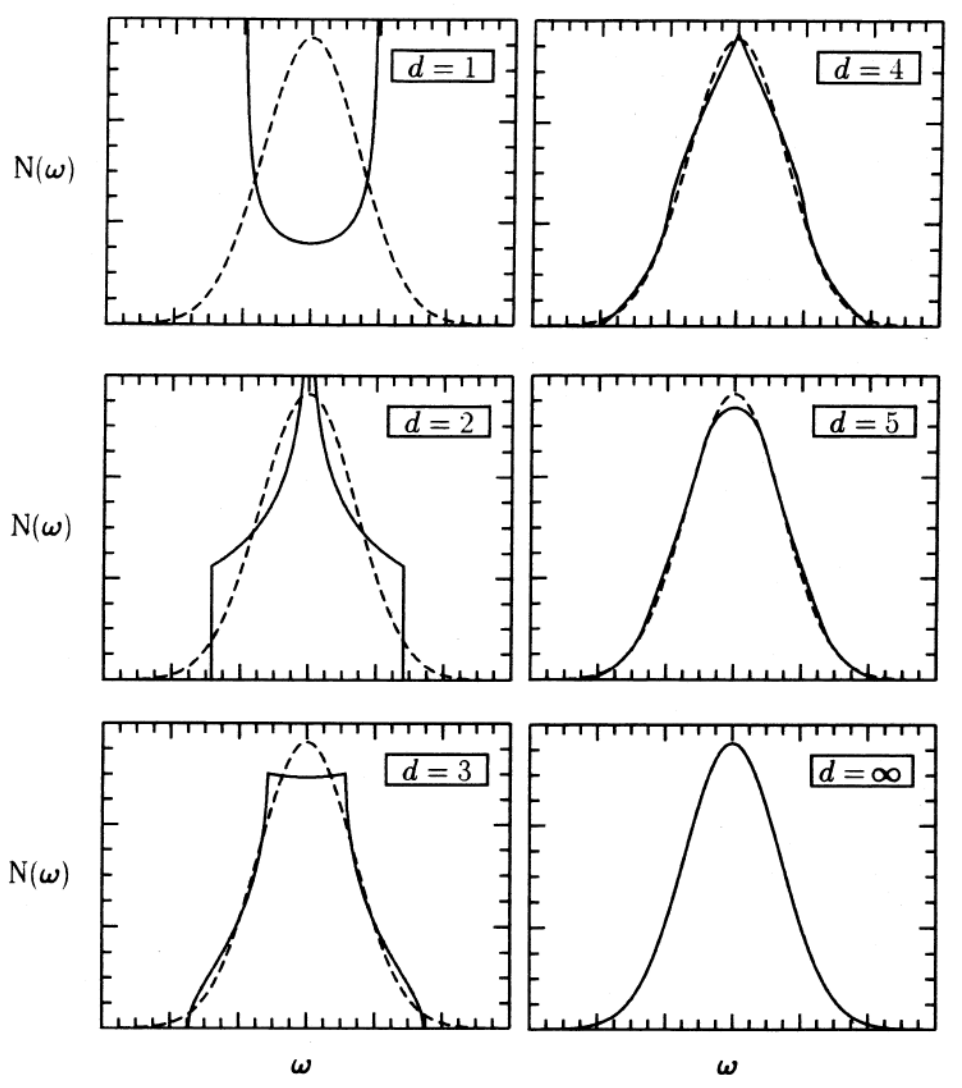

FIGURE 3. Density of states of a tight-binding dispersion relation $\varepsilon_{k}$ on a hypercubic lattice in $d=$ $1,2,3,4,5$ (full lines) as compared to the result for $d=\infty$ (dashed line); from Ref. [11].

$1 / 2 t \sqrt{d}$, while van Hove singularities are due to higher extrema, yielding exponentially small contributions to $N_{d}(\omega)$. Hence, using the scaling (19), one finds for $d \gg 1$

$$
N_{d}(\omega)=\frac{1}{\sqrt{2 \pi} t^{*}} e^{-\frac{1}{2}\left(\omega / t^{*}\right)^{2}}\left\{1-\frac{1}{16 d}\left[\left(\frac{\omega}{t^{*}}\right)^{4}-6\left(\frac{\omega}{t^{*}}\right)^{2}+3\right]+\mathscr{O}\left(\frac{1}{d^{2}}\right)\right\} .
$$

It is interesting to compare $N_{d}(\omega)$ for different $d$ as shown in Fig. 3. For $d \geq 3$ the shapes rapidly approach the $d=\infty$ result; the main difference is that for $d<\infty$ the band has a finite width, while in $d=\infty$ there exist exponentially small tails for all $\omega$.

The scaling (19) expresses the fact that, for some randomly chosen $\boldsymbol{k}$,

$$
\varepsilon_{k} / t \sim \mathscr{O}(\sqrt{d}), d \rightarrow \infty
$$


since $\varepsilon_{k} / t$ is the sum of $d \rightarrow \infty$ many random numbers from the interval $[-1,1]$. Clearly, $\boldsymbol{k}=0$ and $\boldsymbol{k}=(\pi, \ldots, \pi)$ are special values, for which (23) does not hold. However, as long as $\varepsilon_{k}$ appears under an integral these points have zero measure.

Using the relation

$$
\frac{1}{L} \sum_{k} F\left(\varepsilon_{k}\right)=\int_{-\infty}^{\infty} d \omega N(\omega) F(\omega)
$$

where $L$ is the number of lattice sites, the kinetic energy of the non-interacting electrons is found as

$$
E_{\mathrm{kin}}^{0}=\sum_{k, \sigma} \varepsilon_{k} n_{k \sigma}=-2 L t^{* 2} N_{\infty}\left(E_{F}\right)
$$

We see that only the quantum scaling (19) leads to a finite kinetic energy in $d=\infty$. Without this scaling $E_{\mathrm{kin}}^{0}$ would diverge, while the classical scaling $t \rightarrow t^{*} / Z$ would reduce $E_{\text {kin }}^{0}$ to zero ${ }^{4}$.

The interaction term in (4) is seen to be purely local and independent of the surrounding; hence it is independent of the spatial dimension of the system. Consequently, the on-site interaction $U$ need not be scaled. So we see that the scaled Hubbard Hamiltonian

$$
\hat{H}=-\frac{t^{*}}{\sqrt{Z}} \sum_{\left\langle\boldsymbol{R}_{i}, \boldsymbol{R}_{j}\right\rangle} \sum_{\sigma} \hat{c}_{i \sigma}^{+} \hat{c}_{j \sigma}+U \sum_{\boldsymbol{R}_{i}} \hat{n}_{i \uparrow} \hat{n}_{i \downarrow}
$$

has a nontrivial $Z \rightarrow \infty$ limit, where both terms, the kinetic energy and the interaction, are of the same order of magnitude and are thereby able to compete. It is this competition between the two terms which leads to interesting many-body physics (see footnote 4).

The quantum scaling (19) was determined within a $\boldsymbol{k}$ - space formulation. We will now derive the same result within a position-space formulation.

\subsection{Simplifications of the many-body perturbation theory}

The most important consequence of the scaling (19) is the fact that it leads to significant simplifications in the investigation of Hubbard-type lattice models [15, 17, 19-22].

\footnotetext{
${ }^{4}$ To obtain a physically meaningful mean-field theory for a model its internal or free energy has to remain finite in the limit $d$ or $Z \rightarrow \infty$. While for the Ising model the scaling $J \rightarrow \tilde{J} / Z, \tilde{J}=$ const., was rather obvious this is not so for more complicated models. Namely, fermionic or bosonic many-particle systems are usually described by a Hamiltonian consisting of several non-commuting terms, e.g., a kinetic energy and an interaction, each of which is associated with a coupling parameter, usually a hopping amplitude and an interaction, respectively. In such a case the question of how to scale these parameters has no unique answer since this depends on the physical effects one wishes to explore. In any case, the scaling should be performed such that the model remains non-trivial and that its internal or free energy stays finite in the $Z \rightarrow \infty$ limit. By "non-trivial" we mean that not only $\left\langle\hat{H}_{0}\right\rangle$ and $\left\langle\hat{H}_{\text {int }}\right\rangle$, but also the competition between these terms, expressed by $\left\langle\left[\hat{H}_{0}, \hat{H}_{\text {int }}\right]\right\rangle$, should remain finite. In the case of the Hubbard model it would be possible to employ classical scaling for the hopping amplitude, i.e., $t \rightarrow t^{*} / Z, t^{*}=$ const., but then the kinetic energy would be reduced to zero in the limit $d \rightarrow \infty$, making the resulting model uninteresting (but not unphysical) for most purposes. For the bosonic Hubbard model the situation is more subtle due to the occurrence of Bose-Einstein condensation; for a discussion see Ref. [18].
} 
To understand this point better we take a look at the perturbation theory in terms of $U$. At $T=0$ and $U=0$ the kinetic energy (25) may be written as

$$
E_{\mathrm{kin}}^{0}=-t \sum_{\left\langle\boldsymbol{R}_{i}, \boldsymbol{R}_{j}\right\rangle} \sum_{\sigma} g_{i j, \sigma}^{0},
$$

where $g_{i j, \sigma}^{0}=\left\langle\hat{c}_{i \sigma}^{+} \hat{c}_{j \sigma}\right\rangle_{0}$ is the one-particle density matrix. This quantity can also be interpreted as the amplitude for transitions between site $\boldsymbol{R}_{i}$ and $\boldsymbol{R}_{j}$, whose square is proportional to the probability for a particle to hop from $\boldsymbol{R}_{i}$ to $\boldsymbol{R}_{j}$, i.e., $\left|g_{i j, \sigma}^{0}\right|^{2} \sim 1 / Z \sim$ $1 / d$ since $\boldsymbol{R}_{i}$ has $\mathscr{O}(d)$ nearest neighbors. Thus the sum of $\left|g_{i j, \sigma}^{0}\right|^{2}$ over all nearest neighbors must yield a constant. In the limit $d \rightarrow \infty$ we then have

$$
g_{i j, \sigma}^{0} \sim \mathscr{O}\left(\frac{1}{\sqrt{d}}\right), \boldsymbol{R}_{j} N N \text { of } \boldsymbol{R}_{i} .
$$

Since the sum over the NN-sites $\boldsymbol{R}_{j}$ in (27) is of $\mathscr{O}(d)$ the NN-hopping amplitude $t$ must obviously be scaled according to (19) for $E_{\text {kin }}^{0}$ to remain finite in the limit $d, Z \rightarrow \infty$. Hence, as expected, a real-space formulation yields the same results for the required scaling of the hopping amplitude.

The one-particle Green function ("propagator") $G_{i j, \sigma}^{0}(\omega)$ of the non-interacting system obeys the same scaling as $g_{i j, \sigma}^{0}$. This follows directly from its definition

$$
G_{i j, \sigma}^{0}(t) \equiv-\left\langle T \hat{c}_{i \sigma}(t) \hat{c}_{j \sigma}^{+}(0)\right\rangle_{0},
$$

where $T$ is the time ordering operator, and the time evolution of the operators is provided by the Heisenberg representation. The one-particle density matrix is obtained as $g_{i j, \sigma}^{0}=$ $\lim _{t \rightarrow 0^{-}} G_{i j, \sigma}^{0}(t)$. If $g_{i j, \sigma}^{0}$ obeys (28) the one-particle Green function must follow the same scaling at all times since this property does not dependent on the time evolution and the quantum mechanical representation. The Fourier transform $G_{i j, \sigma}^{0}(\omega)$ also preserves this property.

It is important to realize that, although the propagator $G_{i j, \sigma}^{0} \sim 1 / \sqrt{d}$ vanishes for $d \rightarrow \infty$, the particles are not localized, but are still mobile. Indeed, even in the limit $d \rightarrow \infty$ the off-diagonal elements of $G_{i j, \sigma}^{0}$ contribute, since a particle may hop to $d$ nearest neighbors with reduced amplitude $t^{*} / \sqrt{2 d}$. For general $i, j$ one finds [19,23]

$$
G_{i j, \sigma}^{0} \sim \mathscr{O}\left(1 / d^{\left\|\boldsymbol{R}_{i}-\boldsymbol{R}_{j}\right\| / 2}\right)
$$

where $\|\boldsymbol{R}\|=\sum_{n=1}^{d}\left|R_{n}\right|$ is the length of $\boldsymbol{R}$ in the so-called "New York metric" (also called "taxi cab metric", since particles only hop along horizontal or vertical lines, never along a diagonal).

It is the property (30) which is the origin of all simplifications arising in the limit $d \rightarrow \infty$. In particular, it implies the collapse of all connected, irreducible perturbation theory diagrams in position space $[15,17,19]$. This is illustrated in Fig. 4, where a contribution in second-order perturbation theory to the irreducible self-energy, $\Sigma_{i j}^{(2)}$, is shown. 

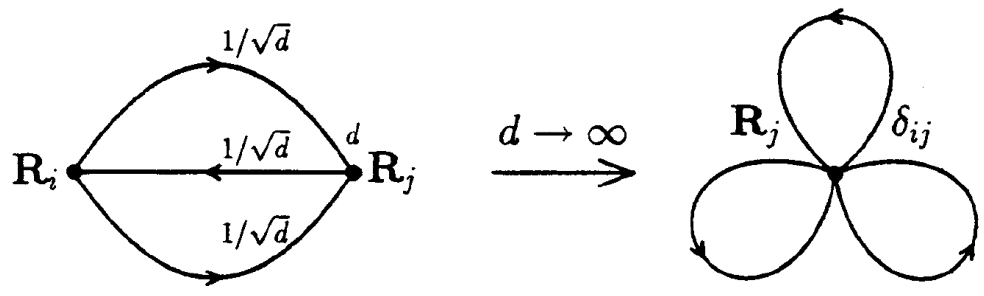

FIGURE 4. Contribution to the irreducible self-energy for the Hubbard model in second-order perturbation theory in $U$, and its collapse in the limit $d \rightarrow \infty$.

In all physically interesting circumstances $\Sigma_{i j}^{(2)}$ will only enter in a sum over $\boldsymbol{R}_{i}$ and $\boldsymbol{R}_{j}$. Then it becomes apparent that $\Sigma_{i j}^{(2)}$ is only of order $1 / \sqrt{d}$ small, unless $i=j$. Namely, for $j \neq i$ the three lines, corresponding to $G_{i j, \sigma}^{0}$, contribute a factor $1 / d^{3 / 2}$, while the sum over NN- sites $\boldsymbol{R}_{j}$ of $\boldsymbol{R}_{i}$ contributes a factor $d$. Only for $i=j$ is the value of $\Sigma_{i j}^{(2)}$ independent of $d$. Hence in the limit $d \rightarrow \infty$ the diagram on the left-hand side of Fig. 4 is equivalent to the "collapsed", petal-shaped diagram on the r.h.s., provided $i=j$; otherwise it is zero. More generally, any two vertices which are connected by more than two separate paths will collapse onto the same site ${ }^{5}$. In particular, the external vertices of any irreducible self-energy diagram are always connected by three separate paths and hence always collapse. As a consequence the full, irreducible self-energy becomes a purely local quantity $[15,17]$ :

$$
\Sigma_{i j, \sigma}(\omega) \stackrel{d \rightarrow \infty}{=} \Sigma_{i i, \sigma}(\omega) \delta_{i j} .
$$

In the paramagnetic phase we may write $\Sigma_{i i, \sigma}(\omega) \equiv \Sigma(\omega)$. The Fourier transform of $\Sigma_{i j, \sigma}$ is seen to become momentum-independent

$$
\Sigma_{\sigma}(\boldsymbol{k}, \omega) \stackrel{d \rightarrow \infty}{\equiv} \Sigma_{\sigma}(\omega)
$$

This leads to tremendous simplifications in all many-body calculations for the Hubbard model and related models. It should be noted that a $\boldsymbol{k}$-independence of $\Sigma$ is sometimes assumed as a convenient approximation ("local approximation") [24-26]. Here we identified the limit where this is indeed exact.

The result expressed in (31b) may equally be obtained by working in $\boldsymbol{k}$-space from the beginning [17]. For this we consider an external vertex where a momentum $k$ enters from outside (see Fig. 5).

Making use of the fact that (i) the Hubbard interaction is momentum independent,

\footnotetext{
${ }^{5}$ Here a "path" is any sequence of lines in a diagram; they are "separate" when they have no lines in common.
} 


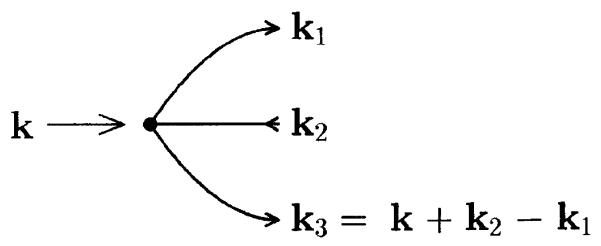

FIGURE 5. Interaction vertex for the Hubbard model with external momentum and frequency $\mathbf{k}=$ $(\boldsymbol{k}, \omega)$.

(ii) the $k$-dependent, free propagator is given by

$$
G_{k}^{0}(\omega)=\frac{1}{\omega-\varepsilon_{k}+\mu} \equiv G^{0}\left(\varepsilon_{k}, \omega\right)
$$

and only depends on $\boldsymbol{k}$ via $\varepsilon_{\boldsymbol{k}}$ (we do not write the spin index explicitly), and (iii) that momenta only enter explicitly in the conservation of momentum at the vertex, we see that the evaluation of the vertex involves the momentum summation

$$
\begin{gathered}
\frac{1}{L^{3}} \sum_{\boldsymbol{k}_{1}, \boldsymbol{k}_{2}, \boldsymbol{k}_{3}} G_{\boldsymbol{k}_{1}}^{0}\left(\omega_{1}\right) G_{\boldsymbol{k}_{2}}^{0}\left(\omega_{2}\right) G_{\boldsymbol{k}_{3}}^{0}\left(\omega_{3}\right) \boldsymbol{\delta}^{*}\left(\boldsymbol{k}-\boldsymbol{k}_{1}+\boldsymbol{k}_{2}-\boldsymbol{k}_{3}\right) \\
\equiv \prod_{i=1}^{3}\left[\int d \varepsilon_{i} G^{0}\left(\varepsilon_{i}, \omega_{i}\right)\right] N_{\boldsymbol{k}}\left(\varepsilon_{1}, \varepsilon_{2}, \varepsilon_{3}\right),
\end{gathered}
$$

where $\omega_{1}-\omega_{2}+\omega_{3}=\omega$ and

$$
\delta^{*}(\boldsymbol{q})=\sum_{\boldsymbol{K}} \delta(\boldsymbol{q}+\boldsymbol{K})=\frac{1}{(2 \pi)^{d}} \sum_{\boldsymbol{R}} e^{i \boldsymbol{q} \cdot\left(\boldsymbol{R}-\boldsymbol{R}_{0}\right)}
$$

is the "Laue-function" which guarantees momentum conservation up to a reciprocal lattice vector $\boldsymbol{K}$. The lattice summation extends over all sites $\boldsymbol{R}$ relative to some origin $\boldsymbol{R}_{0}$ (without loss of generality we may put $\boldsymbol{R}_{0}=0$ ) and couples momenta explicitly. Without this coupling the momentum-summation would be simple because we would be able to use (24). In (33b) we therefore introduced a generalized density of states

$$
N_{k}\left(\varepsilon_{1}, \varepsilon_{2}, \varepsilon_{3}\right)=\frac{1}{L^{3}} \sum_{\boldsymbol{k}_{1}, \boldsymbol{k}_{2}, \boldsymbol{k}_{3}} \delta\left(\varepsilon_{1}-\varepsilon_{\boldsymbol{k}_{1}}\right) \delta\left(\varepsilon_{2}-\varepsilon_{\boldsymbol{k}_{2}}\right) \delta\left(\varepsilon_{3}-\varepsilon_{\boldsymbol{k}_{3}}\right) \boldsymbol{\delta}^{*}\left(\boldsymbol{k}-\boldsymbol{k}_{1}+\boldsymbol{k}_{2}-\boldsymbol{k}_{3}\right),
$$

which is the probability density for $\varepsilon_{i}=\varepsilon_{\boldsymbol{k}_{i}}(i=1,2,3)$ for given $\boldsymbol{k}$. Writing the $\delta$ functions in (35) as a Fourier series (see (21a)) and using (34), one finds in the limit $d=\infty$

$$
N_{k}\left(\varepsilon_{1}, \varepsilon_{2}, \varepsilon_{3}\right)=N_{\infty}\left(\varepsilon_{1}\right) N_{\infty}\left(\varepsilon_{2}\right) N_{\infty}\left(\varepsilon_{3}\right), \quad d=\infty
$$

i. e., for all $\boldsymbol{k}$ the quantity $N_{k}$ factorizes into a product of one-particle DOS's. This is equivalent to replacing the Laue-function in (35) by unity

$$
\delta^{*}(\boldsymbol{q}) \stackrel{d \rightarrow \infty}{=} 1
$$


It effectively means that the momentum conservation constraint may be ignored in $d=\infty$. Defining the position of the interaction vertex in Fig. 5 by $\boldsymbol{R}_{0} \equiv 0$, (37) means that in the lattice sum over $\boldsymbol{R}$ only the local term $\boldsymbol{R}=0$ contributes. This is, once again, the collapse-phenomenon discussed above. Due to the irrelevance of momentum conservation an external momentum $\boldsymbol{k}$ cannot enter into the internal structure of an irreducible self-energy diagram; this makes the irreducible self-energy $\boldsymbol{k}$-independent in $d=\infty$ (see (31b)). Note, however, that the total momentum of a particle must be conserved for the theory to be meaningful.

Due to the simplifications caused by (36) or (31), the most important obstacle for actual diagrammatic calculations in finite dimensions $d \geq 1$, namely the integration over intermediate momenta, is removed in $d=\infty$. While in finite dimensions these integrations lead to untractable technical problems, they become simple in $d=\infty$, since one can replace them by one-dimensional integrations over the DOS.

It should be noted that the limit $d \rightarrow \infty$ does not affect the dynamics of the system at all. Time is always one-dimensional and hence there is no "collapse" in the frequency variables. In spite of the simplifications in position (or momentum) space the problem retains its full dynamics in $d=\infty$.

\subsection{Interactions beyond the on-site interaction}

In the case of more general interactions than the Hubbard interaction, e. g., nearest neighbor interactions such as

$$
\hat{H}_{n n}=\sum_{\left\langle\boldsymbol{R}_{i}, \boldsymbol{R}_{j}\right\rangle} \sum_{\sigma \sigma^{\prime}} V_{\sigma \sigma^{\prime}} \hat{n}_{i \sigma} \hat{n}_{j \sigma^{\prime}}
$$

the interaction constant has to be scaled, too, in the limit $d \rightarrow \infty$. In the case of (38), which has the form of a classical interaction, the "classical" scaling

$$
V_{\sigma \sigma^{\prime}} \rightarrow \frac{V_{\sigma \sigma^{\prime}}^{*}}{Z}
$$

is required. Of course, the propagator still has the dependence (30). The self-energy has the general diagrammatic form shown in Fig. 6.

Due to (39) all contributions, except for the Hartree-term, are found to vanish in $d=\infty$. Hence nonlocal interactions only contribute via their Hartree-contribution, which is purely static. This gives the Hubbard interaction a unique role: of all interactions for fermionic lattice models only the Hubbard interaction remains dynamical in the limit $d \rightarrow \infty[17]$.

\subsubsection{The Hartree approximation revisited}

We are now able to answer the question whether the decoupling leading to the Hartree approximation (13) also becomes exact in the limit $d \rightarrow \infty$ as in the case of the Ising 


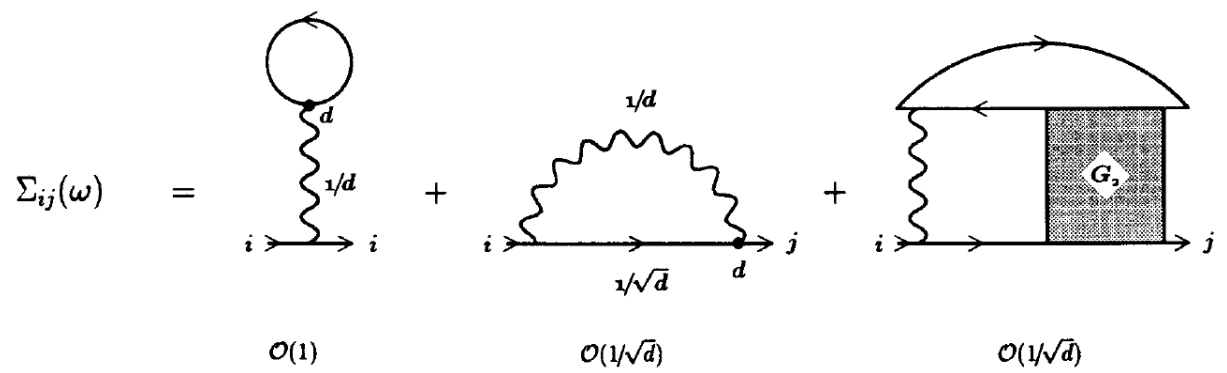

FIGURE 6. Diagrams representing the irreducible self-energy.

model. Using (15) the answer is simple: as long as the on-site interaction in (12) is present, the decoupling (15) cannot become exact in $d \rightarrow \infty$, because for $i=j$ (and hence $\sigma=-\sigma^{\prime}$ ) the r.h.s. of (15) is always of order unity. Namely, at a given site $\boldsymbol{R}_{i}$ the potential, in units of $U$, felt by an electron with spin $\sigma$ is either 0 (if there is no $-\sigma$ spin present) or 1 (if a $-\sigma$ spin is present). These on-site fluctuation effects are not described by the Hartree decoupling approximation. This has been explicitly verified by investigating the thermodynamics of (12) in $d=\infty$ [27]. On the other hand, if the onsite interaction in (12) did not exist (e.g., in the case of spinless fermions) the Hartree approximation (15) would indeed become exact in the limit $d \rightarrow \infty$.

\subsection{One-particle and two-particle propagators}

Due to the $\boldsymbol{k}$-independence of the irreducible self-energy, (31b), the one-particle propagator of an interacting lattice fermion system is given by

$$
G_{k, \sigma}(\omega)=\frac{1}{\omega-\varepsilon_{k}+\mu-\Sigma_{\sigma}(\omega)}
$$

Most importantly, the $\boldsymbol{k}$ dependence of $G_{\boldsymbol{k}}(\omega)$ comes entirely from the energy dispersion $\varepsilon_{k}$ of the non-interacting particles. This means that for a homogeneous system with the propagator

$$
G_{i j, \sigma}(\omega)=L^{-1} \sum_{k} G_{k, \sigma}(\omega) e^{i \boldsymbol{k} \cdot\left(\boldsymbol{R}_{i}-\boldsymbol{R}_{j}\right)}
$$




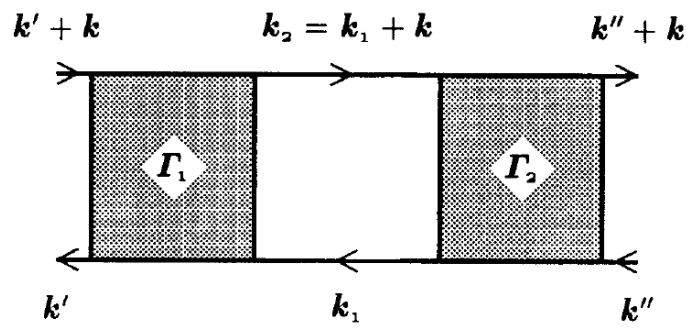

FIGURE 7. Diagrammatic contribution to the two-particle propagator.

its local part, i. e., $G_{i i, \sigma}$, can be calculated in closed form [20]

$$
\begin{aligned}
G_{i i, \sigma}(\omega) & =L^{-1} \sum_{k} G_{k, \sigma}(\omega)=\int_{-\infty}^{\infty} d E \frac{N_{\infty}(E)}{\omega-E+\mu-\Sigma_{\sigma}(\omega)} \\
& =-i \sqrt{\frac{\pi}{2}} \frac{1}{t^{*}} e^{-z^{2}} \operatorname{erfc}(-i z) \\
& \equiv G(\omega),
\end{aligned}
$$

where $z=\left(\omega+\mu-\Sigma_{\sigma}(\omega)\right) / \sqrt{2} t^{*}, N_{\infty}(E)$ is given by (20), and erfc(x) is the error function. The spectral function of the interacting system (often referred to as the DOS as in the non-interacting case) is then given by

$$
A(\omega)=-\frac{1}{\pi} \operatorname{Im} G\left(\omega+i 0^{+}\right)
$$

for $U=0$ one has $A(\omega) \equiv N(\omega)$. So in the paramagnetic phase, where we can ignore the spin index, and in the limit $d \rightarrow \infty$ only two quantities play the most important role: the local propagator $G(\omega)$ and the self-energy $\Sigma(\omega)$.

Concerning the two-particle propagator $G_{2}$ (or correlation functions, etc.) the collapse phenomenon is a little different from the one discussed below (35). Namely, it does not occur for all external momenta $[23,28]$. A typical contribution to $G_{2}$ is shown in Fig. 7, where $\Gamma_{1}, \Gamma_{2}$ are irreducible, momentum independent vertices.

The calculation of this contribution is analogous to that in (33), and involves the $\boldsymbol{k}$ sum over a product of two one-particle propagators, i. e., over $G_{\boldsymbol{k}_{1}}^{0} G_{\boldsymbol{k}_{2}}^{0}$ with $\boldsymbol{k}_{2}=\boldsymbol{k}_{1}+\boldsymbol{k}$. Introducing a generalized DOS

$$
N_{k}\left(\varepsilon_{1}, \varepsilon_{2}\right)=\sum_{\boldsymbol{k}_{1}, \boldsymbol{k}_{2}} \delta\left(\varepsilon_{1}-\varepsilon_{\boldsymbol{k}_{1}}\right) \delta\left(\varepsilon_{2}-\varepsilon_{\boldsymbol{k}_{2}}\right) \delta^{*}\left(\boldsymbol{k}_{2}-\boldsymbol{k}_{1}-\boldsymbol{k}\right)
$$

we find that for $d=\infty$ this quantity only factorizes into $N_{\infty}\left(\varepsilon_{1}\right) N_{\infty}\left(\varepsilon_{2}\right)$ if $\boldsymbol{k} \neq 0$, $\boldsymbol{k} \neq(\pi, \ldots, \pi)$, i. e., when $\varepsilon_{\boldsymbol{k}} \sim \mathscr{O}(1 / \sqrt{d})$. Hence the Bethe-Salpeter ladder collapses for all $\boldsymbol{k}$, except for these two special values. 


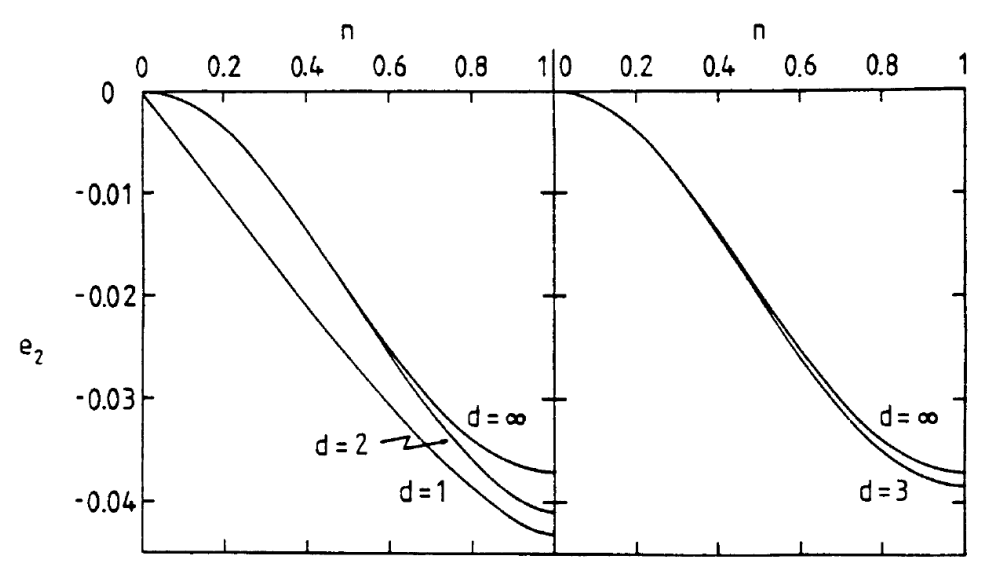

FIGURE 8. Correlation energy for the Hubbard model in second-order perturbation theory in $U$, $e_{2}=E_{c}^{(2)} /\left(2 U^{2} /\left|\bar{\varepsilon}_{0}\right|\right)$, vs. density $n$ for several lattice dimensions. Left: $d=1,2, \infty$; right: $d=3, \infty$. Here $\left|\bar{\varepsilon}_{0}\right|$ is the kinetic energy for $U=0$ and $n=1$; after Ref. [15].

\subsection{Weak-coupling correlation energy for the Hubbard Model}

The correlation energy $E_{c}(U)$ is defined as the energy by which the Hartree-Fock energy is lowered when genuine correlations are included

$$
E_{c}(U):=E_{\text {exact }}(U)-E_{\mathrm{HF}}(U) .
$$

For $d \rightarrow \infty$ second-order Goldstone perturbation theory in the weak interaction $U$ yields the simple expression $\left(n_{\uparrow}=n_{\downarrow}=\frac{1}{2}\right)$ [15]

$$
E_{c}^{(2)}(U)=-U^{2} \int_{0}^{\infty} d \omega e^{2 \omega^{2}} P^{2}\left(E_{F}-\omega\right) P^{2}\left(-E_{F}-\omega\right),
$$

where $P(x)$ is the Gaussian probability function. It should be stressed that for general dimensions the calculation of $E_{c}^{(2)}$ involves $3 d$ momentum integrals over a singular integrand. Analytic calculations are thereby ruled out and even numerical integration techniques becomes very cumbersome. Indeed, the application of Monte-Carlo integration techniques becomes mandatory already for $d \gtrsim 2$. By contrast, the case $d=\infty$ is seen to be the simplest of all dimensions, including $d=1$, since it only demands a one-dimensional integral.

In Fig. $8 E_{c}^{(2)} / U^{2}$ is shown as a function of particle density $n$ for several dimensions $d$. We see that the result for $d=3$, which can only be obtained by considerable effort, is very well approximated by the result for $d=\infty$, which is easily calculated. The differences between $d=1,2$ and $d=3$ are mainly due to the singularities of the DOS in $d=1,2$. 


\subsection{Consequences of the $k$-independence of the self-energy}

We now discuss some more consequences of the $\boldsymbol{k}$-independence of the self-energy as derived by Müller-Hartmann [20]. Let us consider the Hubbard model and concentrate on the paramagnetic phase. That is, we do not wish to discuss the more complicated situation with a broken symmetry for the moment. ${ }^{6}$ In the paramagnetic case at $T=0$ the one-particle propagator (40) takes the form (again we do not write the spin index explicitly)

$$
G_{k}(\omega)=\frac{1}{\omega-\varepsilon_{k}+E_{F}-\Sigma(\omega)} .
$$

In general, even when $\Sigma(\omega)$ is $\boldsymbol{k}$-dependent, the Fermi surface is defined by the $\omega=0$ limit of the denominator of (46) as

$$
\varepsilon_{k}+\Sigma_{k}(0)=E_{F} .
$$

According to Luttinger and Ward [29] the volume within the Fermi surface is not changed by interactions, provided the effect of the latter can be treated in infinite-order perturbation theory (hence no broken symmetry). This is expressed by

$$
n=\sum_{k \sigma} \theta\left[E_{F}-\varepsilon_{k}-\Sigma_{k}(0)\right],
$$

where $n$ is the particle density and $\theta(x)$ is the step function. In general, the $\boldsymbol{k}$-dependence of $\Sigma_{k}(0)$ in (47a) implies that, in spite of (47b), the shape of the Fermi surface of the interacting system will be quite different from that of the non-interacting system (except for the rotationally invariant case $\varepsilon_{\boldsymbol{k}}=f(|\boldsymbol{k}|)$. For lattice fermion models in $d=\infty$, where $\Sigma_{k}(\omega) \equiv \Sigma(\omega)$, (47a) implies that the Fermi surface itself (and hence the volume enclosed) is not changed by interactions. ${ }^{7}$ The Fermi energy is simply shifted uniformly from its non-interacting value $E_{F}^{0}$, i. e., $E_{F}=E_{F}^{0}+\Sigma(0)$, to keep $n$ in (47b) constant. From (42b) we thus conclude that the $\omega=0$ value of the local propagator, $G(0)$, and hence of the spectral function, $A(0)=-\frac{1}{\pi} \operatorname{Im} G\left(i 0^{+}\right)$, is not changed by interactions. ${ }^{8}$ Renormalizations of $N(0)$ can only come from a $k$-dependence of $\Sigma$, i. e., if $\partial \Sigma / \partial \boldsymbol{k} \neq 0$.

For $\omega \rightarrow 0$ the self-energy has the property

$$
\operatorname{Im} \Sigma(\omega) \propto \omega^{2}
$$

which implies quasiparticle (Fermi liquid) behavior. The effective mass

$$
\frac{m^{*}}{m}=1-\left.\frac{d \Sigma}{d \omega}\right|_{\omega=0}=1+\frac{1}{\pi} \int_{-\infty}^{\infty} d \omega \frac{\operatorname{Im} \Sigma\left(\omega+i 0^{-}\right)}{\omega^{2}} \geq 1
$$

\footnotetext{
${ }^{6}$ On bipartite lattices and for nearest neighbor hopping the Hubbard model has a "perfect-nesting instability" at half filling due to $\varepsilon_{\boldsymbol{k}}=-\varepsilon_{\boldsymbol{k}+\boldsymbol{Q}}$ with $\boldsymbol{Q}=(\pi, \ldots, \pi)$, leading to an insulating state with antiferromagnetic correlations in $d \geq 2$. In this case the system is not in a paramagnetic state even for arbitrarily small $U$. However, by including a finite hopping amplitude to next-nearest neighbors the paramagnetic phase becomes stable for small $U$.

${ }^{7}$ In $d=\infty$ the notion of a Fermi surface is made complicated by the fact that in this limit the dispersion $\varepsilon_{k}$ is not a simple smooth function.

${ }^{8}$ This behavior is well-known from the single-impurity Anderson model [30].
} 
is seen to be enhanced. In particular, the momentum distribution

$$
n_{k}=\frac{1}{\pi} \int_{-\infty}^{0} d \omega \operatorname{Im} G_{k}(\omega)
$$

has a discontinuity at the Fermi surface, given by $n_{k_{F}^{-}}-n_{k_{F}^{+}}=\left(m^{*} / m\right)^{-1}$, where $k_{F}^{ \pm}=$ $k_{F} \pm 0^{+}$.

\section{DYNAMICAL MEAN-FIELD THEORY FOR CORRELATED LATTICE FERMIONS}

Itinerant quantum mechanical models such as the Hubbard model and its generalizations are much more complicated than classical, Ising-type models. Generally there do not even exist semiclassical approximations for such models that might serve as a starting point for further investigations. Under such circumstances the construction of a meanfield theory with the comprehensive properties of the Weiss molecular field theory for the Ising model will necessarily be much more complicated, too. As discussed above there do exist well-known mean-field approximation schemes, e. g. Hartree-Fock, random-phase approximation, saddle-point evaluations of path integrals, decoupling of operators. However, these approximations do not provide mean-field theories in the spirit of statistical mechanics, since they are not able to provide a global description of a given model (e.g., the phase diagram, thermodynamics, etc.) in the entire range of input parameters.

Here the limit of high spatial dimensions $d$ or coordination number $Z$ has again been extremely useful [15]. It provides the basis for the construction of a comprehensive mean-field theory for lattice fermions which is diagrammatically controlled and whose free energy has no unphysical singularities. The construction is based on the scaled Hamiltonian (26) and the simplifications in the many-body perturbation theory discussed in Sec. 3.2. There we saw that the local propagator $G(\omega)$, i.e., the amplitude for an electron to return to a lattice site, and the local but dynamical self-energy $\Sigma(\omega)$ are the most important quantities in such a theory. Since the self-energy is a dynamical variable (in contrast to Hartree-Fock theory where it is merely a static potential) the resulting mean-field theory will also be dynamical and can thus describe genuine correlation effects such as the Mott-Hubbard metal-insulator transition.

The self-consistency equations of this dynamical mean-field theory (DMFT) for correlated lattice fermions can be derived in different ways. Nevertheless, all derivations make use of the fact that in the limit of high spatial dimensions Hubbard-type models reduce to a "dynamical single-site problem", where the $d$-dimensional lattice model is effectively described by the dynamics of the correlated fermions on a single site which is embedded in a "bath" provided by the other particles. In the following I will present two different derivations of the single-site action and the self-consistency equations of the DMFT. I start with the approach by Janiš [31, 32] who generalized the coherent potential approximation (CPA) for disordered systems. Then I discuss today's standard derivation developed independently by Georges and Kotliar [33] which is based on the mapping of the lattice problem onto a self-consistent single-impurity Anderson model; 
this approach was also employed by Jarrell [34]. The DMFT equations derived within the CPA approach and the single-impurity approach, respectively, are identical. Nevertheless it is the Anderson-impurity formulation which was immediately adopted by the community since it makes contact with the theory of quantum impurities and Kondo problems; for a review see Ref. [35]. This is a well-understood branch of many-body physics for whose solution efficient numerical codes had been developed already in the 1980's, in particular by making use of the quantum Monte-Carlo (QMC) method [36].

Although the single-impurity based derivation of the DMFT is now the standard method I will present both approaches since it is always instructive to derive a theory in more than one way.

\subsection{Construction of the DMFT by generalizing the coherent potential approximation}

The coherent potential approximation (CPA) is a well-known mean-field theory which was originally developed in the context of disordered systems [37-40]. To be specific let us consider Anderson's tight-binding Hamiltonian" with local ("diagonal”) disorder [41]

$$
\hat{H}_{A}=-\frac{t^{*}}{\sqrt{Z}} \sum_{\left\langle\boldsymbol{R}_{i}, \boldsymbol{R}_{j}\right\rangle} \hat{c}_{i}^{+} \hat{c}_{j}+\sum_{i} V_{i} \hat{n}_{i},
$$

where $V_{i}$ is a random variable drawn from some distribution function $P\left(V_{i}\right)$. The electrons described by (49) do not interact. Therefore we deal with the problem of a single particle moving through a random medium. (Since there is no spin dependence one can suppress the spin index altogether and simply work with a spinless fermion). The problem is made complicated by the randomness. It requires one to calculate the average of a physical quantity $X$ (which is a function of all site energies $V_{i}$ ) with respect to $P\left(V_{i}\right)$. In particular, the arithmetic average is obtained by

$$
\langle X\rangle_{\mathrm{av}}:=\prod_{\boldsymbol{R}_{i}} \int d V_{i} P\left(V_{i}\right) X\left(V_{1}, \ldots, V_{L}\right) .
$$

One may now proceed as follows:

1. The actual random medium, given by the local potentials $V_{i}$, is thought to be replaced exactly by an (unknown) effective medium, described by a complex, frequency-dependent self-energy; this defines the self-energy.

2. Since the effective medium is required to yield an exact description of the random medium, we may remove the medium at a site $\boldsymbol{R}_{i}$, replace it by an actual potential $V_{i}$ and then demand that, upon averaging, the scattering caused by the perturbation of the medium due to $V_{i}$ vanishes identically. The self-consistency condition expressed in the last step determines the previously unknown self-energy.

\footnotetext{
${ }^{9}$ Here the kinetic energy is already scaled according to (19) to allow for the limit $Z \rightarrow \infty$ to be taken, in which case the CPA becomes exact (see Sec. 4.1.1.).
} 
Let $G_{i j}(z) \equiv G$ be the Green function of the electron in the random medium, with $z$ as a complex frequency and $G_{0}$ as the unperturbed Green function; we suppress site-indices for the moment [42]. The Lippmann-Schwinger equation for $G$ is given by

$$
G=G_{0}+G_{0} V G \quad \text { or } \quad G_{0}^{-1} G=1+V G .
$$

We now introduce a self-energy $\Sigma_{i j}(z) \equiv \Sigma$ into (51), which plays the role of an unknown potential

$$
\left(G_{0}^{-1}-\Sigma\right) G=1+(V-\Sigma) G
$$

and demand

$$
\langle G\rangle_{\mathrm{av}}=\left(G_{0}^{-1}-\Sigma\right)^{-1} .
$$

Multiplication of (52) by $\langle G\rangle_{\text {av }}$ yields

$$
G=\langle G\rangle_{\mathrm{av}}+\langle G\rangle_{\mathrm{av}}(V-\Sigma) G
$$

Here $V-\Sigma$ is a new scattering potential, whose effect can be described by a $T$-matrix via

$$
G=\langle G\rangle_{\mathrm{av}}+\langle G\rangle_{\mathrm{av}} T\langle G\rangle_{\mathrm{av}},
$$

where

$$
T=\frac{V-\Sigma}{1-(V-\Sigma)\langle G\rangle_{\mathrm{av}}} .
$$

Averaging of (55) yields

$$
\langle T\rangle_{\mathrm{av}}=0 \text {, }
$$

which is a self-consistent equation for $\Sigma$. Eq. (57) demands that $\Sigma$ is determined in such a way that the scattering due to the perturbation $V-\Sigma$ vanishes. If (57) could be solved exactly the entire problem would be solved. However, an exact solution is usually not possible (an exception is the Lloyd model where $P\left(V_{i}\right)$ is given by a Lorentzian), so that an approximation has to be made to proceed further. At this stage CPA assumes the self-energy to be site-diagonal

$$
\Sigma_{i j}(\omega)=\Sigma(\omega) \delta_{i j}
$$

i. e., to be homogeneous. Eq. (58) is equivalent to a single-site approximation and corresponds to step 2 in the construction of the CPA described below (50). Since $\Sigma(\omega)$ is homogeneous it is a $\boldsymbol{k}$ independent, but frequency dependent potential and thereby only adds to the frequency dependence of $G_{0}^{-1}$, i. e., the averaged propagator is simply given by the unperturbed propagator with shifted frequency :

$$
\langle G\rangle_{\mathrm{av}}=G_{i i}^{0}(z-\Sigma(z)) .
$$

For all sites $\boldsymbol{R}_{i}$ the condition (57) therefore reduces to $\left\langle T_{i}\right\rangle_{\mathrm{av}}=0$, i. e.,

$$
\left\langle\frac{V_{i}-\Sigma(z)}{1-\left(V_{i}-\Sigma(z)\right) G_{i i}^{0}(z-\Sigma(z))}\right\rangle_{\mathrm{av}}=0
$$



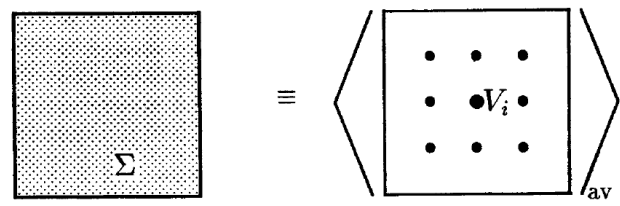

(a)
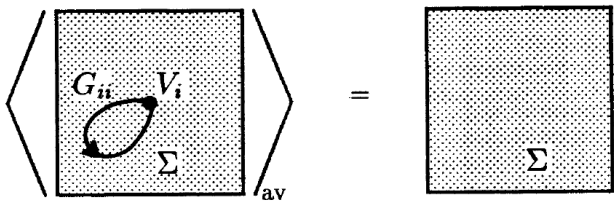

(b)

FIGURE 9. (a) The random medium, described by local potentials $V_{i}$, is replaced by an unknown, but exact, effective potential $\Sigma$. (b) By demanding the average scattering from a single site with potential $V_{i}-\Sigma$ to vanish, $\Sigma$ is determined self-consistently; from Ref. [11].

where

$$
G_{i i}^{0}(z)=\int_{-\infty}^{\infty} d \omega \frac{N(\omega)}{z-\omega}
$$

is the local propagator, with $N(\omega)$ as the DOS of the unperturbed system. Eq. (60) implies that in the effective medium the average scattering by a single site ("impurity") vanishes. The single-site aspect underlying the CPA may therefore be visualized as shown in Fig. 9 [39].

The CPA and its results have many attractive features:

(i) CPA is a non-perturbative, but very simple and self-consistent theory;

(ii) it may be considered the best single-site approximation for the disorder problem as can be inferred from the above derivation;

(iii) it has the so-called Herglotz-properties $\Sigma(z)=\Sigma^{*}\left(z^{*}\right)$ and $\operatorname{Im}(z-\Sigma(z)) \gtrless$ for $z<0$ [44], which implies that it has the correct analytic properties (positive DOS, etc);

(iv) it leads to very good qualitative and even quantitative results for the one-particle properties of disordered systems. The latter is true even in dimensions $d \lesssim 3$ and for parameter values of the disorder strength and the impurity concentration where CPA cannot be linked to perturbation theory. These properties have made CPA the most widely used approximation scheme for disordered systems.

\subsubsection{CPA and the limit $d \rightarrow \infty$}

The single-site aspect of the CPA outlined above and, in particular, the property (58), indicate that the CPA will become exact in the limit of high coordination number $Z$. 
Indeed, by investigating the moments of the electronic DOS it was observed that $1 / Z$ appears as a "hidden" small parameter, which governs the size of the corrections to the CPA moments [45]. However, by considering only the moments one cannot draw conclusions about the validity of the CPA itself. ${ }^{10}$ Using the scaling of $t$ in (19) one can show [46] that (58), and hence CPA itself, indeed becomes exact for $Z \rightarrow \infty$, irrespective of the lattice structure. In other words, CPA solves the disorder Hamiltonian (49) exactly in the limit $Z \rightarrow \infty$. This finding explains why for $Z<\infty$ the CPA can be so successful even for intermediate values of the disorder strength and impurity concentration, i. e., when perturbation theory in these parameters is no longer justified. In fact, we now see that there is an additional small parameter, namely $1 / Z$, which allows for a perturbation expansion that is independent of the values of the input parameters. In view of the existence of a small parameter $1 / Z$, CPA is seen to be a controlled mean-field theory. It is therefore not so surprising that the CPA often gives qualitatively and quantitatively correct results even in dimensions $d \lesssim 3$.

\subsubsection{Alternative derivation of $C P A$}

I will now show that the CPA can also be derived from a variational principle [43]. To this end the coherent potential, i. e., the self-energy, will be determined from the averaged free-energy functional $\Omega$ of the corresponding single-site problem [47]. This field-theoretical approach has the advantage that it can be generalized to treat interacting lattice models, such as Hubbard-type models and disorder models on the same basis (see Sec. 4.1.3). Furthermore, the physical idea behind this single-site theory is very transparent and may be explained in terms of the following simple picture [43]. To calculate the averaged free energy corresponding to a single site $\boldsymbol{R}_{i}$ of the medium we have to determine the energy density

$$
\left\langle\Omega_{i}\right\rangle_{\mathrm{av}} \equiv \frac{\langle\Omega\rangle_{\mathrm{av}}}{L},
$$

where $L$ is the number of lattice sites. To this end we consider the second step of the CPA strategy outlined below (50) and drawn schematically in Fig. 10:

(i) we start from the homogeneous, effective medium with free energy density $\Omega_{\text {med }} / L$;

(ii) we remove the medium at site $\boldsymbol{R}_{i}$, i. e., subtract a corresponding energy $\Omega_{i}$; and

(iii) replace it by a site with a bare potential $V_{i}$, i. e., add a corresponding averaged energy $\left\langle\Omega_{i}^{\text {bare }}\right\rangle_{\text {av }}$; so we have

$$
\frac{\langle\Omega\rangle_{\mathrm{av}}}{L}=\frac{\Omega_{\mathrm{med}}}{L}-\Omega_{i}+\left\langle\Omega_{i}^{\mathrm{bare}}\right\rangle_{\mathrm{av}}
$$

\footnotetext{
${ }^{10}$ In fact, in Ref. [45] the kinetic energy was not scaled according to $t \rightarrow t^{*} / \sqrt{Z}$, but the bandwidth was kept constant, corresponding to classical scaling $\left(t \rightarrow t^{*} / Z\right)$. In this case the $Z \rightarrow \infty$ limit does not lead to CPA at all, but becomes trivial.
} 


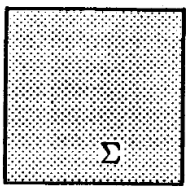

(a)

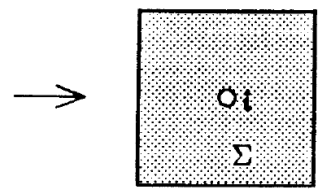

(b)

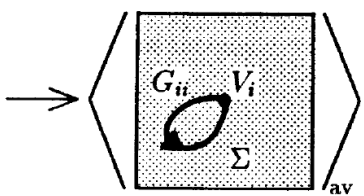

(c)

FIGURE 10. Steps to construct the exact averaged free energy functional in $d=\infty$ : (a) Homogeneous effective medium, (b) the medium is removed at site $\boldsymbol{R}_{i}$, (c) the cavity in the medium at site $\boldsymbol{R}_{i}$ is filled with the actual potential $V_{i}$. The probability amplitude for a particle from the medium to be at site $\boldsymbol{R}_{i}$ (more precisely: the return amplitude) is given by the local propagator $G_{i i}$; from Ref. [11].

To calculate the contributions in (63) we make use of the trace-log formula for the free energy density which in the non-interacting case (superscript 0 ) reads [48]

$$
\frac{\Omega^{0}}{L}=-T \operatorname{tr} \sum_{k} \ln \left[G_{k}^{0}\left(i \omega_{n}\right)\right]^{-1} .
$$

Here $G_{k}^{0}$ is given by (32), $\omega_{n}=(2 n+1) \pi T$ are the Matsubara frequencies and the trace operation implies

$$
\operatorname{tr}=\sum_{\sigma} \sum_{n=-\infty}^{\infty}
$$

where in the present problem the spin is unimportant. Using (59), i. e.,

$$
\left\langle G_{i i}(z)\right\rangle_{\mathrm{av}}=G_{i i}^{0}(z-\Sigma(z))
$$

we have

$$
\begin{aligned}
\frac{\Omega_{\mathrm{med}}}{L} & =-T \operatorname{tr} \sum_{k} \ln \left[G_{k}^{0}\left(i \omega_{n}-\Sigma\left(i \omega_{n}\right)\right)\right]^{-1} \\
& =-T \operatorname{tr} \int_{-\infty}^{\infty} d E N(E) \ln \left[i \omega_{n}-\Sigma\left(i \omega_{n}\right)+\mu-E\right]
\end{aligned}
$$

and

$$
\Omega_{i}=-T \operatorname{tr} \ln \left[G_{i i}^{0}\left(i \omega_{n}-\Sigma\left(i \omega_{n}\right)\right)\right]^{-1}=-T \operatorname{tr} \ln \left[\left\langle G_{i i}\left(i \omega_{n}\right)\right\rangle_{\mathrm{av}}\right]^{-1},
$$

where $G_{i i}^{0}$ is the local, unperturbed propagator needed to remove the medium at site $\boldsymbol{R}_{i}$. Finally, $\left\langle\Omega_{i}^{\text {bare }}\right\rangle_{\text {av }}$ can be obtained from

$$
\Omega_{i}^{\text {bare }}=-T \ln \mathscr{Z}_{i}^{\text {bare }},
$$


where the local partition function is determined by the action $S_{i}^{\text {bare }}$ as [48]

$$
\mathscr{Z}_{i}^{\text {bare }}=\int D c D c^{*} \exp \left[-S_{i}^{\text {bare }}\left\{c, c^{*}\right\}\right]
$$

Here $c, c^{*}$ are Grassmann (anti-commuting) variables [48, 49]. The action is given by

$$
S_{i}^{\mathrm{bare}}=-\operatorname{tr} c_{n}^{*}\left[\left\langle G_{i i}\left(i \omega_{n}\right)\right\rangle_{\mathrm{av}}\right]^{-1} c_{n}+\operatorname{tr} c_{n}^{*}\left(V_{i}-\Sigma\left(i \omega_{n}\right)\right) c_{n},
$$

where $c_{n} \equiv c\left(i \omega_{n}\right), c_{n}^{*} \equiv c^{*}\left(i \omega_{n}\right)$, and the two terms correspond to the kinetic and potential energy, respectively. We note that the local potential at site $\boldsymbol{R}_{i}$ is measured relative to the surrounding effective medium $\Sigma\left(i \omega_{n}\right)$. Since (71) is the expression for a non-interacting system (bilinear dependence on $c$ and $c^{*}$ ) the functional intergral in (70) is trivial to perform and yields, together with the trace-log formula $\operatorname{det} A=\exp (\operatorname{tr} \ln A)$,

$$
\begin{aligned}
\ln \mathscr{Z}_{i}^{\text {bare }} & =\operatorname{tr} \ln \left\{\left[\left\langle G_{i i}\left(i \omega_{n}\right)\right\rangle_{\mathrm{av}}\right]^{-1}-V_{i}+\Sigma\left(i \omega_{n}\right)\right\} \\
& =\operatorname{tr} \ln \left(\mathscr{G}^{-1}\left(i \omega_{n}\right)-V_{i}\right),
\end{aligned}
$$

where we introduced an effective local propagator $\mathscr{G}$ by

$$
\mathscr{G}^{-1}\left(i \omega_{n}\right) \equiv\left[\left\langle G_{i i}\left(i \omega_{n}\right)\right\rangle_{\mathrm{av}}\right]^{-1}+\Sigma\left(i \omega_{n}\right) .
$$

The $\mathscr{G}\left(i \omega_{n}\right)$ propagator describes the coupling between the medium and the site $\boldsymbol{R}_{i}$.

The averaged free energy, which is a functional of $\mathscr{G}^{-1}(\Sigma)$, then follows as

$$
\begin{array}{r}
\langle\Omega\rangle_{\mathrm{av}}=-L T \operatorname{tr}\left\{\int d E N(E) \ln \left[i \omega_{n}+\mu-\Sigma\left(i \omega_{n}\right)-E\right]\right. \\
\left.-\ln \left(\mathscr{G}^{-1}\left(i \omega_{n}\right)-\Sigma\left(i \omega_{n}\right)\right)+\left\langle\ln \left(\mathscr{G}^{-1}\left(i \omega_{n}\right)-V_{i}\right)\right\rangle_{\mathrm{av}}\right\} .
\end{array}
$$

By taking the variational derivative of (73) w.r.t. $\Sigma$, i. e., using the stationarity condition

$$
\frac{\delta\langle\Omega\rangle_{\mathrm{av}}}{\delta \mathscr{G}^{-1}}=0
$$

we obtain

$$
\frac{1}{\mathscr{G}^{-1}\left(i \omega_{n}\right)-\Sigma\left(i \omega_{n}\right)}=\left\langle\frac{1}{\mathscr{G}^{-1}\left(i \omega_{n}\right)-V_{i}}\right\rangle_{\mathrm{av}} .
$$

Together with (66) and (72c) this equation is seen to be identical to the self-consistent Eq. (60) for $\Sigma\left(i \omega_{n}\right)$. Given a value $\mathscr{G}^{-1}\left(i \omega_{n}\right)$ we obtain $\Sigma\left(i \omega_{n}\right)$ from (75), which determines a new value $\mathscr{G}^{-1}\left(i \omega_{n}\right)=\left[G_{i i}^{0}\left(\left(i \omega_{n}\right)-\Sigma\left(i \omega_{n}\right)\right)\right]^{-1}+\Sigma\left(i \omega_{n}\right)$ and so on. Eq. (75) expresses particularly clearly the single-site aspect of the CPA, as well as the role of $\Sigma\left(i \omega_{n}\right)$ as a homogeneous effective potential that describes the effect of the original random medium in the averaged system. 


\subsubsection{Generalization of the CPA approach to interacting systems}

The CPA was extensively used in the 1970's to investigate disordered systems. It was also applied to interacting models, e. g. the Hubbard model, by first transforming the model (approximately) to a random alloy problem ("alloy analogy") [40].

A new approach to the CPA, which makes use of field-theoretical functional integral techniques in connection with explicit diagrammatic perturbation theory, was initiated by Janiš $[47,50]$. Thereby the range of applicability of the CPA was extended to interacting lattice systems (spin systems and itinerant systems). In this generalized singlesite approach the free-energy functional can be derived in closed form. The derivation is based on the general scheme for the construction of conserving approximations by Baym [51], i. e., the free energy $\Omega\{\Sigma\}$, which is a functional of $\Sigma$, is written as

$$
\beta \Omega\{\Sigma\}=\Phi\{G\}-\operatorname{tr}(\Sigma G)-\operatorname{tr} \ln \left[\left(G^{0}\right)^{-1}-\Sigma\right] .
$$

Here $G_{k \sigma}^{0}(\omega)$ is the free propagator, (32), and $G$ is the full propagator which is determined by

$$
\frac{\partial \Omega}{\partial \Sigma}=0
$$

The quantity $\Phi\{G\}$ is obtained from a self-consistent perturbation expansion in $G$ for the self-energy [51], the latter being defined by

$$
\frac{\delta \Phi}{\delta G}=\Sigma
$$

Hence $\Omega$ is a functional of $\Sigma$ only. To define $\Omega$ unambiguously one has to impose the boundary condition $\Phi=0$ for $\Sigma=0$. The construction of $\Omega\{\Sigma\}$ amounts to the construction of the functional

$$
\Lambda\{\Sigma, G\}=\Phi\{G\}-\operatorname{tr}(\Sigma G) .
$$

In a single-site theory, where the self-energy is purely local, $\Lambda$ is fully determined by $\Sigma$ and the local part of $G$. In this case the construction of $e^{-\beta \Lambda}$ reduces to a single-site problem, which can be performed explicitly $[47,50]$.

Due to the insight gained from the investigation of the large- $d$ limit for fermionic lattice systems one can now conclude that the generalized CPA approach $[47,50]$ becomes exact in $d=\infty$ [31], just as the CPA for disordered systems and other single-site theories discussed so far become exact in this limit. In particular, this field-theoretical approach can be used to derive the exact free energy for fermionic models in $d=\infty$ $[31,32,43]$. This leads to a comprehensive, controlled mean-field theory even for interacting fermionic models which is conceptually identical to the CPA for disordered systems or to the Weiss theory for the Ising model. Of course, this theory is necessarily much more complicated in detail than the previous mean-field theories since we now deal with a dynamical single-site problem in a fermionic bath.

The physical idea behind the approach is the same as that described in the last subsection in connection with the CPA. Let us consider the motion of a particle on 
a lattice in $d=\infty$. The interaction with the other particles affects the motion. This change is exactly described by a yet unknown complex, dynamical field $\Sigma_{\sigma}(\omega)$. Hence the original system with its bare interactions has been exactly replaced by an effective medium; the latter is simply a system of non-interacting, itinerant electrons moving in a complex, homogeneous coherent potential $\Sigma_{\sigma}(\omega)$.

\subsubsection{Exact free energy functional for the Hubbard model in $d=\infty$ and the self-consistency equations}

We will now use the generalized CPA described above to construct an exact expression for the free energy of the Hubbard model in $d=\infty[31,32,43]$. We proceed as in the case

of disordered systems (see Sec. 4.1.2 and Fig. 10), with $V_{i}$ replaced by $\hat{v}_{i \sigma}=U \hat{n}_{i,-\sigma}$, but we do not have to perform any impurity average now. The single-site free energy density $\Omega / L$ is given by

$$
\frac{\Omega}{L}=\frac{\Omega_{\mathrm{med}}}{L}-\Omega_{i}+\Omega_{i}^{\text {bare }} .
$$

Using the analog of (66)

$$
G_{i i, \sigma}(z)=G_{i i, \sigma}^{0}\left(z-\Sigma_{\sigma}(z)\right)
$$

the first two terms are given by (see (66), (68))

$$
\begin{gathered}
\frac{\Omega_{\mathrm{med}}}{L}=-T \operatorname{tr} \sum_{k} \ln \left[G_{k, \sigma}^{0}\left(i \omega_{n}-\Sigma_{\sigma}\left(i \omega_{n}\right)\right)\right]^{-1} \\
\Omega_{i}=-T \operatorname{tr} \ln \left[G_{i i, \sigma}^{0}\left(i \omega_{n}-\Sigma_{\sigma}\left(i \omega_{n}\right)\right)\right]^{-1}=-T \operatorname{tr} \ln \left[G_{i i, \sigma}\left(i \omega_{n}\right)\right]^{-1} .
\end{gathered}
$$

The contribution $\Omega_{i}^{\text {bare }}$, obtained by replacing the medium on site $\boldsymbol{R}_{i}$ by the actual, bare interaction (here: the Hubbard interaction) is again given by (69) and (70), where the single-site action is now given by $[31,43]$

$$
\begin{aligned}
S_{i}^{\text {bare }} & =-\operatorname{tr} c_{\sigma, n}^{*}\left[G_{i i, \sigma}\left(i \omega_{n}\right)\right]^{-1} c_{\sigma, n} \\
& +\left[U \int_{0}^{\beta} d \tau c_{\uparrow}^{*}(\tau) c_{\uparrow}(\tau) c_{\downarrow}^{*}(\tau) c_{\downarrow}(\tau)-\operatorname{tr} c_{\sigma, n}^{*} \Sigma_{\sigma}\left(i \omega_{n}\right) c_{\sigma, n}\right] .
\end{aligned}
$$

This expression has the same form as (71), but the spin-dependence of the problem has now been taken into account explicitly $\left(c_{n} \rightarrow c_{\sigma, n}\right)$ and the local one-particle potential $V_{i}$ has been replaced by the Hubbard interaction between up and down-spins.

In analogy with (72c) we now introduce an effective local propagator $\mathscr{G}_{\sigma}\left(i \omega_{n}\right)$ by

$$
\mathscr{G}_{\sigma}^{-1}\left(i \omega_{n}\right) \equiv\left[G_{\sigma}\left(i \omega_{n}\right)\right]^{-1}+\Sigma_{\sigma}\left(i \omega_{n}\right)
$$

where $G_{\sigma}\left(i \omega_{n}\right) \equiv G_{i i, \sigma}\left(i \omega_{n}\right)$. The effective propagator $\mathscr{G}_{\sigma}\left(i \omega_{n}\right)$ again describes the coupling between the medium and the interaction-site $\boldsymbol{R}_{i}$. Note, that sites do not communicate with one another but only via the effective medium. With (84) the action (83) takes 
the form

$$
S_{i}^{\text {bare }}\left\{c_{\sigma}, c_{\sigma}^{*} ; \mathscr{G}_{\sigma}^{-1}\right\}=-\operatorname{tr} c_{\sigma, n}^{*} \mathscr{G}_{\sigma}^{-1}\left(i \omega_{n}\right) c_{\sigma, n}+U \int_{0}^{\beta} d \tau c_{\uparrow}^{*}(\tau) c_{\uparrow}(\tau) c_{\downarrow}^{*}(\tau) c_{\downarrow}(\tau) .
$$

In (70) the partition function $\mathscr{Z}_{i}^{\text {bare }}$ is given by an integral over anticommuting Grassmann variables. It may be transformed into a conventional functional integral over real, commuting variables by rewriting the Hubbard interaction in (85) using the Hubbard-Stratonovich transformation

$$
\begin{gathered}
\exp \left\{-U \int_{0}^{\beta} d \tau c_{\uparrow}^{*}(\tau) c_{\uparrow}(\tau) c_{\downarrow}^{*}(\tau) c_{\downarrow}(\tau)\right\}=\int D \eta D \xi \exp \left\{-\frac{1}{2 \beta} \int_{0}^{\beta} d \tau\left[\eta^{2}(\tau)+\xi^{2}(\tau)\right.\right. \\
\left.\left.-i \sqrt{2 U \beta}\left\{\xi(\tau)\left[c_{\uparrow}^{*}(\tau) c_{\uparrow}(\tau)+c_{\downarrow}^{*}(\tau) c_{\downarrow}(\tau)\right]-i \eta(\tau)\left[c_{\uparrow}^{*}(\tau) c_{\uparrow}(\tau)-c_{\downarrow}^{*}(\tau) c_{\downarrow}(\tau)\right]\right\}\right]\right\} .
\end{gathered}
$$

This is equivalent to the standard operator identity

$$
\hat{n}_{i \uparrow} \hat{n}_{i \downarrow}=\frac{1}{4}\left[\left(\hat{n}_{i \uparrow}+\hat{n}_{i \downarrow}\right)^{2}-\left(\hat{n}_{i \uparrow}-\hat{n}_{i \downarrow}\right)^{2}\right]
$$

for the Hubbard interaction, where the two terms on the right-hand side correspond to charge and spin fluctuations, respectively. In (86) the fluctuations are described by real fluctuating fields $\xi(\tau)$ and $\eta(\tau)$, respectively. Now that the interaction problem has been rewritten in terms of non-interacting particles in the presence of infinitely many fluctuating fields, the integration over the Grassmann variables in the expression for the partition function can be performed explicitly, yielding

$$
\mathscr{Z}_{i}^{\text {bare }}=\int D \eta D \xi \exp \left[-S_{i}^{\text {bare }}\left\{\eta, \xi ; \mathscr{G}_{\sigma}^{-1}\right\}\right]
$$

where now

$$
\begin{aligned}
S_{i}^{\text {bare }}\left\{\eta, \xi ; \mathscr{G}_{\sigma}^{-1}\right\} & =\frac{1}{2} \sum_{v=-\infty}^{\infty}\left(\xi_{v}^{2}+\eta_{v}^{2}\right) \\
& -\operatorname{tr} \ln \left[\hat{\mathscr{G}}_{\sigma}^{-1}-\sqrt{\frac{U}{2 \beta}}(\sigma \hat{\eta}+i \hat{\xi})\right]
\end{aligned}
$$

with $(\hat{\xi})_{m n}=\xi_{m-n},(\hat{\eta})_{m n}=\eta_{m-n}$ and $\left(\hat{\mathscr{G}}_{\sigma}^{-1}\right)_{m n}=\delta_{m n}\left[\mathscr{G}_{\sigma}\left(i \omega_{n}\right)\right]^{-1}$.

Making use of the relation (64) and combining the three contributions in (72), the total free energy is then found as

$\Omega=-L T \operatorname{tr}\left\{\int d \omega N(\omega) \ln \left[i \omega_{n}+\mu-\Sigma_{\sigma}-\omega\right]-\ln \left(\mathscr{G}_{\sigma}^{-1}-\Sigma_{\sigma}\right)\right\}-L T \ln \mathscr{Z}_{i}^{\text {bare }}$

While $\Omega$ was originally a functional of $\Sigma_{\sigma}$ it is now understood as a functional of $\left[\mathscr{G}_{\sigma}\left(\Sigma_{\sigma}\right)\right]^{-1}$. The stationarity condition (74) is then

$$
\frac{\delta \Omega}{\delta \mathscr{G}_{\sigma}^{-1}}=0
$$


which leads to

$$
\begin{aligned}
\frac{1}{\left[\mathscr{G}_{\sigma}\left(i \omega_{n}\right)\right]^{-1}-\Sigma_{\sigma}\left(i \omega_{n}\right)} & =\frac{1}{\mathscr{Z}_{i}^{\text {bare }}} \int D c D c^{*}\left(c_{\sigma, n} c_{\sigma, n}^{*}\right) \exp \left[-S_{i}^{\text {bare }}\left\{c_{\sigma}, c_{\sigma}^{*} ; \mathscr{G}_{\sigma}^{-1}\right\}\right](92 \mathrm{a}) \\
& =\frac{1}{\mathscr{Z}_{i}^{\text {bare }}} \int D \eta D \xi\left(\frac{1}{\hat{\mathscr{G}}_{\sigma}^{-1}-\sqrt{\frac{U}{2 \beta}}(\sigma \hat{\eta}+i \hat{\xi})}\right)_{n n} \\
& \times \exp \left[-S_{i}^{\text {bare }}\left\{\eta, \xi ; \mathscr{G}_{\sigma}^{-1}\right\}\right] \\
& \equiv\left\langle\left(\frac{1}{\hat{\mathscr{G}}_{\sigma}^{-1}-\sqrt{\frac{U}{2 \beta}}(\sigma \hat{\eta}+i \hat{\xi})}\right)_{n n}\right\rangle_{\eta, \xi}
\end{aligned}
$$

with $\langle X\rangle_{\eta, \xi}=\left(\int D \eta D \xi X \exp \left[-S_{i}^{\text {bare }}\right]\right) / \mathscr{Z}_{i}$ bare . The right-hand side of (92a) is nothing but the very definition of the local propagator $G_{i i, \sigma}\left(i \omega_{n}\right) \equiv G_{\sigma}\left(i \omega_{n}\right)$ in terms of the action $S_{i}^{\text {bare }}$. Eqs. (92), together with

$$
\left[\mathscr{G}_{\sigma}(z)\right]^{-1}=\left[G_{\sigma}^{0}\left(z-\Sigma_{\sigma}(z)\right)\right]^{-1}+\Sigma_{\sigma}(z)
$$

and the local action (85) provide an exact, self-consistent set of equations for $\Sigma_{\sigma}$ (or $\mathscr{G}_{\sigma}$ ) for the Hubbard model in $d=\infty$ [31-33]. In the paramagnetic phase two quantities are seen to play the most important role: the local propagator $G_{\sigma}(\omega)$ and the self-energy $\Sigma(\omega)$; this is illustrated in Fig. 11.

Although the interaction problem is mathematically much more complicated than the analogous expression (38) for the disorder problem without interactions it can, in principle, again be solved by iteration: for given $\mathscr{G}_{\sigma}^{-1}$ we obtain $\Sigma_{\sigma}$ from (92), which yields a new $\mathscr{G}_{\sigma}^{-1}$ via (93), etc. The exact local propagator is then provided by $G_{i i, \sigma}=\left(\mathscr{G}_{\sigma}^{-1}-\Sigma_{\sigma}\right)^{-1}$. As in the disorder case $G_{i i, \sigma}$ is completely expressed in terms of effective, averaged quantities.

The expression used in (92c) brings out particularly clearly the similarities and differences between the (on-site) interaction problem and the analogous expression (75) for the disorder case without interactions:

(i) on the left-hand side of (92) the self-energy $\Sigma$ appears again as a (homogeneous) effective medium, which is obtained exactly from the original system by some averaging process;

(ii) however, this average is very different in the two cases: in the disorder problem it involves an integration over the actual disorder potentials $V_{i}$ with a given disorder distribution $P\left(V_{i}\right)$, while in (92) it demands an integration over infinitely many fluctuating (random) fields $\eta, \xi$, which simulate the actual interaction;

(iii) the latter integration leads to a highly non-trivial coupling of the energies, i. e., Matsubara frequencies $\omega_{n}$ (note, that this coupling even exists in the static limit, i. e., for $\eta_{v}=\xi_{v}=0$ for $v \neq 0$ ), while in the disorder problem the corresponding Eq. (75) is diagonal in the frequency. This shows clearly that, although the interaction between electrons on different lattice sites has been reduced to an interaction of electrons with 


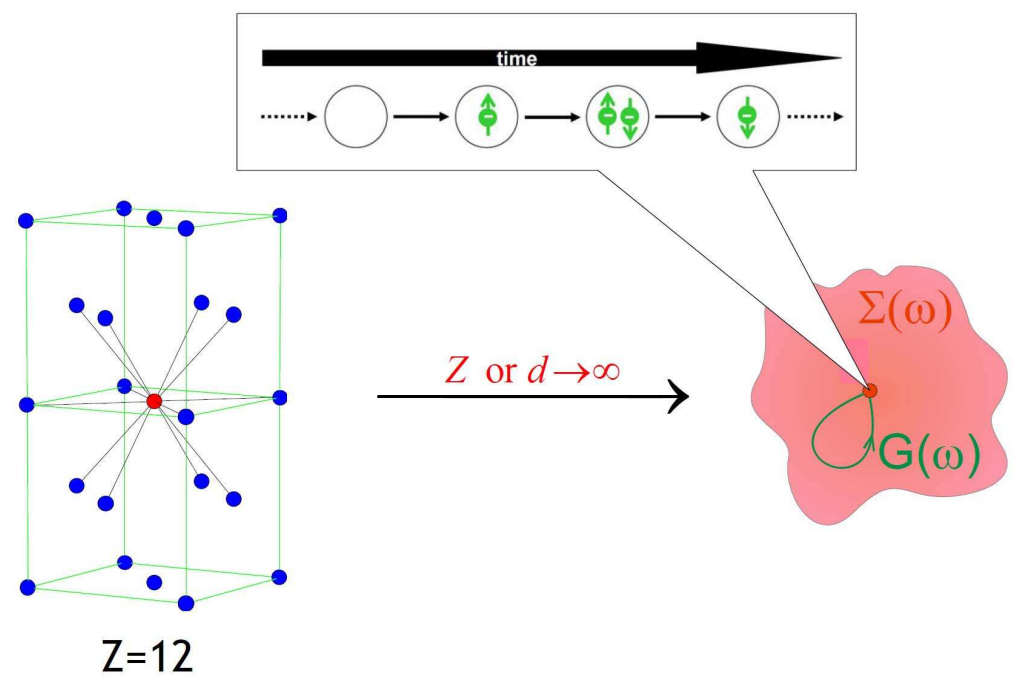

FIGURE 11. In the limit $Z \rightarrow \infty$ the Hubbard model effectively reduces to a dynamical single-site problem, which may be viewed as a lattice site embedded in a dynamical mean field. Electrons may hop from the mean field onto this site and back, and interact on the site as in the original Hubbard model (see Fig. 1). The local propagator $G(\omega)$ (i.e., the return amplitude) and the dynamical self-energy $\Sigma(\omega)$ of the surrounding mean field play the main role in this limit. The quantum dynamics of the interacting electrons is still described exactly.

a mean field, the dynamics of the latter interaction is still non-trivial. Once more we observe that the many-body nature of the Hubbard model survives even in $d=\infty$, making an analytic evaluation of the local propagator $G_{\sigma}$ from (92), (93) generally impossible.

\subsection{Construction of the DMFT as a self-consistent single-impurity Anderson model}

Following the presentation of Georges, Kotliar, Krauth and Rozenberg [52] the dynamical mean-field equations will now be derived using the so-called cavity method. This derivation starts by removing one lattice site together with its bonds from the rest of the lattice. The remaining lattice, which now contains a cavity, is replaced by a particle bath which plays the role of the dynamical mean field (see Fig. 12). So far the derivation and the underlying physical picture coincides with that of the CPA approach described in the previous section. Now comes a new, physically motivated idea: the bath is coupled, via a hybridization, to the cavity. The resulting problem then amounts to the 


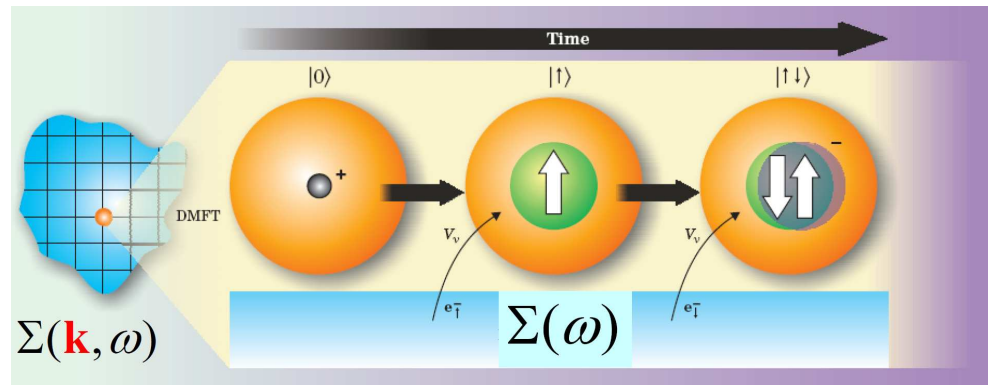

FIGURE 12. The DMFT can be viewed as the mapping of the $d$-dimensional Hubbard model with self-energy $\Sigma(\boldsymbol{k}, \omega)$ onto a single site described by a single-impurity Anderson model which hybridizes with the bath provided by the other electrons [52]. In this local problem the self-energy $\Sigma(\omega)$ is now $\boldsymbol{k}$ independent; after Ref. [53].

solution of an effective single-impurity Anderson model where the degrees of freedom of the bath, represented by an appropriate hybridization function, have to be determined self-consistently.

To be specific, let us consider the partition function in the grand canonical ensemble

$$
\mathscr{Z}=\int \prod_{i \sigma} D c_{i \sigma}^{*} D c_{i \sigma} \exp \left[-S\left\{c_{i \sigma}^{*}, c_{i \sigma}\right\}\right] .
$$

The action $S\left\{c_{i \sigma}^{*}, c_{i \sigma}\right\}$ for the Hubbard model is given by

$$
\begin{array}{r}
S\left\{c_{i \sigma}^{*}, c_{i \sigma}\right\}=\int_{0}^{\beta} d \tau\left[\sum_{i \sigma} c_{i \sigma}^{*}(\tau)\left(\frac{\partial}{\partial \tau}-\mu\right) c_{i \sigma}(\tau)-\sum_{i j \sigma} t_{i j} c_{i \sigma}^{*}(\tau) c_{j \sigma}(\tau)\right. \\
\left.+\sum_{i} U c_{i \uparrow}^{*}(\tau) c_{i \uparrow}(\tau) c_{i \downarrow}^{*}(\tau) c_{i \downarrow}(\tau)\right],
\end{array}
$$

where again as in Sec. 4.1.2 we use Grassman variables $c_{i \sigma}^{*}, c_{i \sigma}$. We split the action $S$ into three parts

$$
S=S_{0}+\Delta S+S^{(0)},
$$

where $S_{0}$ is the part containing only variables on site 0

$$
S_{0}=\int_{0}^{\beta} d \tau\left[\sum_{\sigma} c_{0 \sigma}^{*}(\tau)\left(\frac{\partial}{\partial \tau}-\mu\right) c_{0 \sigma}(\tau)+U c_{0 \uparrow}^{*}(\tau) c_{0 \uparrow}(\tau) c_{0 \downarrow}^{*}(\tau) c_{0 \downarrow}(\tau)\right],
$$

$\Delta S$ contains the hoppings between site 0 and other sites of the lattice $i \neq 0$

$$
\Delta S=-\int_{0}^{\beta} d \tau \sum_{i \sigma}\left[t_{i 0} c_{i \sigma}^{*}(\tau) c_{0 \sigma}(\tau)+t_{0 i} c_{0 \sigma}^{*}(\tau) c_{i \sigma}(\tau)\right]
$$


and the rest, which we denote by $S^{(0)}$, is the part of the action with the site 0 and its bonds removed, i.e., with $i, j \neq 0$

$$
\begin{array}{r}
\left.S^{(0)=\int_{0}^{\beta} d \tau\left[\sum _ { i \neq 0 \sigma } c _ { i \sigma } ^ { * } ( \tau ) \left(\frac{\partial}{\partial \tau}-\right.\right.}-\mu\right) c_{i \sigma}(\tau)-\sum_{i j \neq 0 \sigma} t_{i j} c_{i \sigma}^{*}(\tau) c_{j \sigma}(\tau) \\
\left.+U \sum_{i \neq 0} c_{i \uparrow}^{*}(\tau) c_{i \uparrow}(\tau) c_{i \downarrow}^{*}(\tau) c_{i \downarrow}(\tau)\right] .
\end{array}
$$

Let us now rewrite the partition function $\mathscr{Z}$ as

$$
\begin{array}{r}
\mathscr{Z}=\int \prod_{\sigma} D c_{0 \sigma}^{*} D c_{0 \sigma} \exp \left[-S_{0}\left\{c_{0 \sigma}^{*}, c_{0 \sigma}\right\}\right] \\
\times \int \prod_{i \neq 0 \sigma} D c_{i \sigma}^{*} D c_{i \sigma} \exp \left[-S^{(0)}\left\{c_{i \sigma}^{*}, c_{i \sigma}\right\}\right] \exp \left[-\Delta S\left\{c_{0 \sigma}^{*}, c_{0 \sigma}, c_{i \sigma}^{*}, c_{i \sigma}\right\}\right]
\end{array}
$$

and use the following ensemble average

$$
\langle X\rangle_{(0)} \equiv \frac{1}{\mathscr{Z}(0)} \int \prod_{i \neq 0 \sigma} D c_{i \sigma}^{*} D c_{i \sigma} X \exp \left[-S^{(0)}\left\{c_{i \sigma}^{*}, c_{i \sigma}\right\}\right]
$$

taken with respect to $S^{(0)}$ (the action where the site $i=0$ is excluded), with $\mathscr{Z}^{(0)}$ being the corresponding partition function. Then the partition function reads

$$
\begin{aligned}
\mathscr{Z}=\mathscr{Z}^{(0)} & \int \prod_{\sigma} D c_{0 \sigma}^{*} D c_{0 \sigma} \exp \left[-S_{0}\left\{c_{0 \sigma}^{*}, c_{0 \sigma}\right\}\right] \\
& \times\left\langle\exp \left[-\Delta S\left\{c_{0 \sigma}^{*}, c_{0 \sigma}, c_{i \sigma}^{*}, c_{i \sigma}\right\}\right]\right\rangle_{(0)} .
\end{aligned}
$$

In the next step we expand the second exponent with respect to the action $\Delta S$. As a result we obtain a formally infinite series with all possible many-particle correlation functions, i.e.,

$$
\begin{array}{r}
\mathscr{Z}=\mathscr{Z}^{(0)} \int \prod_{\sigma} D c_{0 \sigma}^{*} D c_{0 \sigma} \exp \left[-S_{0}\left\{c_{0 \sigma}^{*}, c_{0 \sigma}\right\}\right]\left(1-\int_{0}^{\beta} d \tau\langle\Delta S(\tau)\rangle_{(0)}\right. \\
\left.+\frac{1}{2 !} \int_{0}^{\beta} d \tau_{1} \int_{0}^{\beta} d \tau_{2}\left\langle\Delta S\left(\tau_{1}\right) \Delta S\left(\tau_{2}\right)\right\rangle_{(0)}+\cdots\right)
\end{array}
$$

where we used $\Delta S \equiv \int_{0}^{\beta} d \tau \Delta S(\tau)$. In the fermionic case only the correlation functions with equal number of $c$ and $c^{*}$ are non-zero. The lowest order term is second order and reads

$$
\begin{array}{r}
\frac{1}{2 !} \int_{0}^{\beta} d \tau_{1} \int_{0}^{\beta} d \tau_{2}\left\langle\Delta S\left(\tau_{1}\right) \Delta S\left(\tau_{2}\right)\right\rangle_{(0)} \\
=\frac{1}{2 !} \int_{0}^{\beta} d \tau_{1} \int_{0}^{\beta} d \tau_{2} \sum_{\sigma} \sum_{j, k \neq 0}\left[t_{j 0} t_{0 k}\left\langle c_{j \sigma}^{*}\left(\tau_{1}\right) c_{k \sigma}\left(\tau_{2}\right)\right\rangle_{(0)} c_{0 \sigma}\left(\tau_{1}\right) c_{0 \sigma}^{*}\left(\tau_{2}\right)\right. \\
\left.+t_{0 j} t_{k 0}\left\langle c_{j \sigma}\left(\tau_{1}\right) c_{k \sigma}^{*}\left(\tau_{2}\right)\right\rangle_{(0)} c_{0 \sigma}^{*}\left(\tau_{1}\right) c_{0 \sigma}\left(\tau_{2}\right)\right] .
\end{array}
$$


Higher-order terms are obtained similarly. The above expression can be rewritten with the use of one-particle correlation function

$$
G_{j k \sigma}^{(0)}\left(\tau_{1}-\tau_{2}\right)=-\left\langle T_{\tau} c_{j \sigma}\left(\tau_{1}\right) c_{k \sigma}^{*}\left(\tau_{2}\right)\right\rangle_{(0)}
$$

and takes the form

$$
\begin{array}{r}
\frac{1}{2 !} \int_{0}^{\beta} d \tau_{1} \int_{0}^{\beta} d \tau_{2}\left\langle\Delta S\left(\tau_{1}\right) \Delta S\left(\tau_{2}\right)\right\rangle_{(0)} \\
=-\int_{0}^{\beta} d \tau_{1} \int_{0}^{\beta} d \tau_{2} \sum_{\sigma} \sum_{j, k \neq 0} t_{j 0} t_{k 0} G_{j k \sigma}^{(0)}\left(\tau_{1}-\tau_{2}\right) c_{0 \sigma}^{*}\left(\tau_{1}\right) c_{0 \sigma}\left(\tau_{2}\right) .
\end{array}
$$

Higher-order terms can be written in a similar way with the use of n-particle correlation functions.

A non-trivial limit $d \rightarrow \infty$ is obtained by scaling the hopping amplitudes $t_{i j}$ as described in Sec. 3.2. For example, in the second-order contribution to the partition function, (106), the hopping amplitudes must be scaled with $Z^{\left\|\boldsymbol{R}_{0}-\boldsymbol{R}_{j}\right\| / 2}$ because the oneparticle correlation functions are proportional to $1 / Z^{\left\|\boldsymbol{R}_{0}-\boldsymbol{R}_{j}\right\| / 2}$ as discussed in Sec 3.2. In the calculation of higher-order terms we find that all connected higher-order terms vanish at least as $\mathscr{O}(1 / Z)$. Consequently, in the $Z \rightarrow \infty$ limit only the contribution $G_{j k \sigma}^{(0)}$, or disconnected contributions made of products of $G_{j k \sigma}^{(0)}$ 's remain. Applying the linkedcluster theorem and collecting only connected contributions in the exponential function one obtains the local action

$$
\begin{array}{r}
S_{\mathrm{loc}}=\left[\int_{0}^{\beta} d \tau \sum_{\sigma} c_{0 \sigma}^{*}(\tau)\left(\frac{\partial}{\partial \tau}-\mu\right) c_{0 \sigma}(\tau)+U \int_{0}^{\beta} d \tau c_{0 \uparrow}^{*}(\tau) c_{0 \uparrow}(\tau) c_{0 \downarrow}^{*}(\tau) c_{0 \downarrow}(\tau)\right. \\
\left.+\int_{0}^{\beta} d \tau_{1} \int_{0}^{\beta} d \tau_{2} \sum_{\sigma} \sum_{j, k \neq 0} t_{j 0}^{*} t_{k 0}^{*} G_{j k \sigma}^{(0)}\left(\tau_{1}-\tau_{2}\right) c_{0 \sigma}^{*}\left(\tau_{1}\right) c_{0 \sigma}\left(\tau_{2}\right)\right],
\end{array}
$$

where the rescaled hoppings are denoted with a star. Introducing the hybridization function

$$
\Delta_{\sigma}\left(\tau_{1}-\tau_{2}\right)=-\sum_{i, j \neq 0} t_{i 0}^{*} t_{j 0}^{*} G_{i j \sigma}^{(0)}\left(\tau_{1}-\tau_{2}\right),
$$

and employing the free ("Weiss") mean-field propagator $\mathscr{G}_{\sigma}$ one can express the DMFT local action in the following form (here the site index $i=0$ is omitted for simplicity)

$$
\begin{array}{r}
S_{\mathrm{loc}}=-\int_{0}^{\beta} d \tau_{1} \int_{0}^{\beta} d \tau_{2} \sum_{\sigma} c_{\sigma}^{*}\left(\tau_{1}\right) \mathscr{G}_{\sigma}^{-1}\left(\tau_{1}-\tau_{2}\right) c_{\sigma}\left(\tau_{2}\right) \\
+U \int_{0}^{\beta} d \tau c_{\uparrow}^{*}(\tau) c_{\uparrow}(\tau) c_{\downarrow}^{*}(\tau) c_{\downarrow}(\tau),
\end{array}
$$

where

$$
\mathscr{G}_{\sigma}^{-1}\left(\tau_{1}-\tau_{2}\right)=-\left(\frac{\partial}{\partial \tau_{1}}-\mu\right) \delta_{\tau_{1} \tau_{2}}-\Delta_{\sigma}\left(\tau_{1}-\tau_{2}\right)
$$


Finally, we need the relation between the Green function $G_{i j \sigma}^{(0)}\left(\tau-\tau^{\prime}\right)$ where the site $i=0$ is removed and the full lattice Green function, i.e.,

$$
G_{i j \sigma}^{(0)}=G_{i j \sigma}-G_{i 0 \sigma} G_{00 \sigma}^{-1} G_{0 j \sigma},
$$

which holds for a general lattice.

In order to obtain the full solution of the lattice problem it is convenient to express the relation between the local Green function $G_{00 \sigma} \equiv G_{\sigma}$ and the dynamical ("Weiss") mean field $\mathscr{G}_{\sigma}^{-1}$ in the form of a Dyson equation ${ }^{11}$

$$
\left[G_{\sigma}\left(i \omega_{n}\right)\right]^{-1}=\mathscr{G}_{\sigma}^{-1}\left(i \omega_{n}\right)-\Sigma_{\sigma}\left(i \omega_{n}\right)=i \omega_{n}+\mu-\Delta_{\sigma}\left(i \omega_{n}\right)-\Sigma_{\sigma}\left(i \omega_{n}\right) .
$$

Then the lattice Green function (in $\boldsymbol{k}$-space) $G_{\boldsymbol{k} \sigma}\left(i \omega_{n}\right)$ is given by

$$
G_{k \sigma}\left(i \omega_{n}\right)=\frac{1}{i \omega_{n}-\varepsilon_{k}+\mu-\Sigma_{\sigma}\left(i \omega_{n}\right)} .
$$

After performing the so-called lattice Hilbert transform we recover the local Green function

$$
G_{\sigma}\left(i \omega_{n}\right)=\sum_{k} G_{k \sigma}\left(i \omega_{n}\right)=\sum_{k} \frac{1}{i \omega_{n}-\varepsilon_{k}+\mu-\Sigma_{\sigma}\left(i \omega_{n}\right)} .
$$

After analytic continuation to real frequencies the local (" $k$ averaged") propagator reads

$$
G_{k \sigma}(\omega)=\frac{1}{\omega-\varepsilon_{k}+\mu-\Sigma_{\sigma}(\omega)} .
$$

It is very important to realize that although the DMFT corresponds to an effectively local problem, the propagator $G_{k}(\omega)$ is a momentum-dependent quantity. Namely, it depends on the momentum through the dispersion $\varepsilon_{k}$ of the non-interacting electrons, but there is no additional momentum-dependence through the self-energy, since this quantity is strictly local within the DMFT.

The set of self-consistent equations (109), (110), (112), (114) can be solved iteratively. In each step one solves the single-impurity problem given by the action (109), then one finds the new self-energy from the Dyson equation (112) and the new dynamical mean field from (114) and (112). The single-impurity problem is still a complicated manybody interacting problem which cannot, in general, be solved exactly.

\subsection{Solution of the DMFT self-consistency equations}

The dynamics of the full Hubbard model, (4), was found to remain complicated even in the limit $d \rightarrow \infty$ because of the purely local nature of the interaction. Hence an

\footnotetext{
${ }^{11}$ It should be noted that, in principle, any one of the local functions $\Sigma_{\sigma}\left(i \omega_{n}\right), \mathscr{G}_{\sigma}^{-1}\left(i \omega_{n}\right)$, or $\Delta_{\sigma}\left(i \omega_{n}\right)$ can be viewed as a "dynamical mean field" acting on particles on a site, since they all appear in the bilinear term of the local action (109).
} 
exact, analytic evaluation of the self-consistent set of equations (92), (93) for the local propagator $G_{\sigma}$ or the effective propagator $\mathscr{G}_{\sigma}\left(i \omega_{n}\right)$ is not possible. Exact evaluations are only feasible when there is no coupling between the frequencies. This is the case, for example, in the Falicov-Kimball model [54], which was solved analytically by Brandt and Mielsch [21] soon after the introduction of the $d \rightarrow \infty$ limit [15]. A valuable semianalytic approximation is provided by the so-called iterated perturbation theory (IPT) [33], [55], [52].

Solutions of the general DMFT self-consistency equations require extensive numerical methods, in particular quantum Monte Carlo techniques [34], [56, 57], [52], the numerical renormalization group [58, 59], exact diagonalization [60-62], [52] and other techniques, whose discussion requires a separate series of lectures; here I refer the reader to the reviews quoted above.

It quickly turned out that the DMFT is a powerful tool for the investigation of electronic systems with strong correlations. It provides a non-perturbative and thermodynamically consistent approximation scheme for finite-dimensional systems which is particularly valuable for the study of intermediate-coupling problems where perturbative techniques fail [63], [52, 64], [53], [65, 66].

In the remaining part of these lecture notes I shall discuss several applications of the DMFT to problems involving electronic correlations. In particular, I will address the Mott-Hubbard metal-insulator transition, and explain the connection of the DMFT with band-structure methods - the LDA+DMFT scheme - which is the first comprehensive framework for the $a b$ initio investigation of correlated electron materials.

\section{THE MOTT-HUBBARD METAL-INSULATOR TRANSITION}

The correlation induced transition between a paramagnetic metal and a paramagnetic insulator, referred to as "Mott-Hubbard metal-insulator transition (MIT)", is one of the most intriguing phenomena in condensed matter physics [67-69]. This transition is a consequence of the competition between the kinetic energy of the electrons and their local interaction $U$. Namely, the kinetic energy prefers the electrons to move (a wave effect) which leads to doubly occupied sites and thereby to interactions between the electrons (a particle effect). For large values of $U$ the doubly occupied sites become energetically very costly. The system may reduce its total energy by localizing the electrons. Hence the Mott transition is a localization-delocalization transition, demonstrating the particle-wave duality of electrons.

Mott-Hubbard MITs are, for example, found in transition metal oxides with partially filled bands near the Fermi level. For such systems band theory typically predicts metallic behavior. The most famous example is $\mathrm{V}_{2} \mathrm{O}_{3}$ doped with $\mathrm{Cr}$ [70-72]. In particular, in $\left(\mathrm{V}_{0.96} \mathrm{Cr}_{0.04}\right)_{2} \mathrm{O}_{3}$ the metal-insulator transition is of first order below $T=380 \mathrm{~K}$ [71], with discontinuities in the lattice parameters and in the conductivity. However, the two phases remain isostructural.

Making use of the half-filled, single-band Hubbard model (4) the Mott-Hubbard MIT was studied intensively in the past [5, 67-69, 72]. Important early results were obtained by Hubbard [73] within a Green function decoupling scheme, and by Brinkman and Rice [74] who employed the Gutzwiller variational method [4, 75], both at zero tem- 
perature $^{12}$. Hubbard's approach yields a continuous splitting of the band into a lower and upper Hubbard band, but cannot describe quasiparticle features. By contrast, the Gutzwiller-Brinkman-Rice approach (for a review see Ref. [77]) gives a good description of the low-energy, quasiparticle behavior, but cannot reproduce the upper and lower Hubbard bands. In the latter approach the MIT is signalled by the disappearance of the quasiparticle peak.

To solve this problem the DMFT has been extremely valuable since it provided detailed insights into the nature of the Mott-Hubbard MIT for all values of the interaction $U$ and temperature $T[52,78],[53]$.

\subsection{DMFT and the three-peak structure of the spectral function}

The Mott-Hubbard MIT is monitored by the spectral function $A(\omega)=-\frac{1}{\pi} \operatorname{Im} G(\omega+$ $\left.i 0^{+}\right)$of the correlated electrons ${ }^{13}$; here we follow the discussion of Refs. [79], [53], [80]. The change of $A(\omega)$ obtained within the DMFT for the one-band Hubbard model (4) at $T=0$ and half filling $(n=1)$ as a function of the Coulomb repulsion $U$ (measured in units of the bandwidth $W$ of non-interacting electrons) is shown in Figs. 13 and 14. While Fig. 13 is a schematic picture of the evolution of the spectrum when the interaction is increased, Fig. 14 shows actual numerical results obtained by the NRG $[58,80]$. Here magnetic order is assumed to be suppressed ("frustrated").

While at small $U$ the system can be described by coherent quasiparticles whose DOS still resembles that of the free electrons, the spectrum in the Mott insulator state consists of two separate incoherent "Hubbard bands" whose centers are separated approximately by the energy $U$. The latter originate from atomic-like excitations at the energies $\pm U / 2$ broadened by the hopping of electrons away from the atom. At intermediate values of $U$ the spectrum then has a characteristic three-peak structure as in the single-impurity Anderson model, which includes both the atomic features (i.e., Hubbard bands) and the narrow quasiparticle peak at low excitation energies, near $\omega=0$. This corresponds to a strongly correlated metal. The structure of the spectrum (lower Hubbard band, quasiparticle peak, upper Hubbard band) is quite insensitive to the specific form of the DOS of the non-interacting electrons.

The width of the quasiparticle peak vanishes for $U \rightarrow U_{\mathrm{c} 2}(T)$. The "Luttinger pinning" at $\omega=0$ [20] is clearly observed. On decreasing $U$, the transition from the insulator to the metal occurs at a lower critical value $U_{\mathrm{c} 1}$, where the gap vanishes.

It is important to note that the three-peak spectrum originates from a lattice model with only one type of electrons. This is in contrast to the single-impurity Anderson model whose spectrum shows very similar features, but is due to two types of electrons,

\footnotetext{
12 The Gutzwiller variational method $[4,75]$ consists of the choice of a simple projected variational wave function ("Gutzwiller wave function") and a semi-classical evaluation of expectation values in terms of this wave function ("Gutzwiller approximation"). As shown by Metzner and Vollhardt [76], [11] the Gutzwiller approximation becomes exact in the limit $d \rightarrow \infty$. This result initiated the investigations of the Hubbard model in $d \rightarrow \infty$ [15]

13 In the following we only consider the paramagnetic phase.
} 


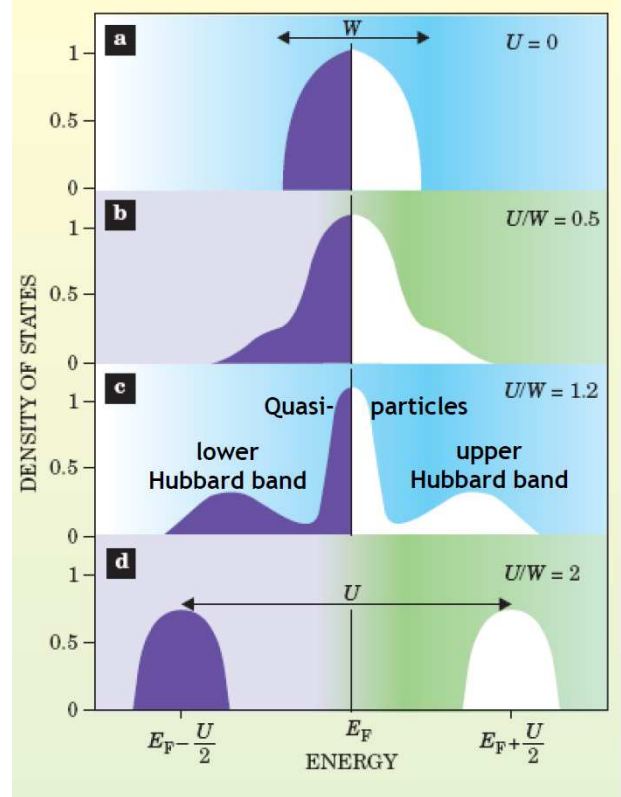

FIGURE 13. Evolution of the spectral function ("density of states") of the Hubbard model in the paramagnetic phase at half filling. a) non-interacting case, b) for weak interactions there is only little transfer of spectral weight away from the Fermi energy, c) for strong interactions a typical three-peak structure consisting of coherent quasiparticle excitations close to the Fermi energy and incoherent lower and upper Hubbard bands is clearly seen, d) above a critical interaction the quasiparticle peak vanishes and the system is insulating, with two well-separated Hubbard bands remaining; after Ref. [53].

namely the localized orbital at the impurity site and the free conduction band. Therefore the screening of the magnetic moment which gives rise to the Kondo effect in impurity systems has a different origin in lattice systems. Namely, as explained by the DMFT, the same sort of electrons provide both the local moments and the electrons which screen these moments $[35,52]$.

The evolution of the spectral function of the half-filled frustrated Hubbard model at finite temperatures, $T=0.0276 \mathrm{~W}$, is shown in Fig. 15. This temperature is above the temperature of the critical point so that there is no real transition but only a crossover from a metallic-like to an insulating-like solution. The height of the quasiparticle peak at the Fermi energy is no longer fixed at its zero temperature value. This is due to a finite value of the imaginary part of the self-energy. The spectral weight of the quasiparticle peak is seen to be gradually redistributed and shifted to the upper (lower) edge of the lower (upper) Hubbard band. The inset of Fig. 15 shows the $U$-dependence of the value of the spectral function at zero frequency $A(\omega=0)$. For higher values of $U$ the spectral density at the Fermi level is still finite and vanishes only in the limit $U \rightarrow \infty$ (or for $T \rightarrow 0$, provided that $U>U_{\mathrm{c} 2}(T=0)$ ).

For the insulating phase DMFT predicts the filling of the Mott-Hubbard gap with 


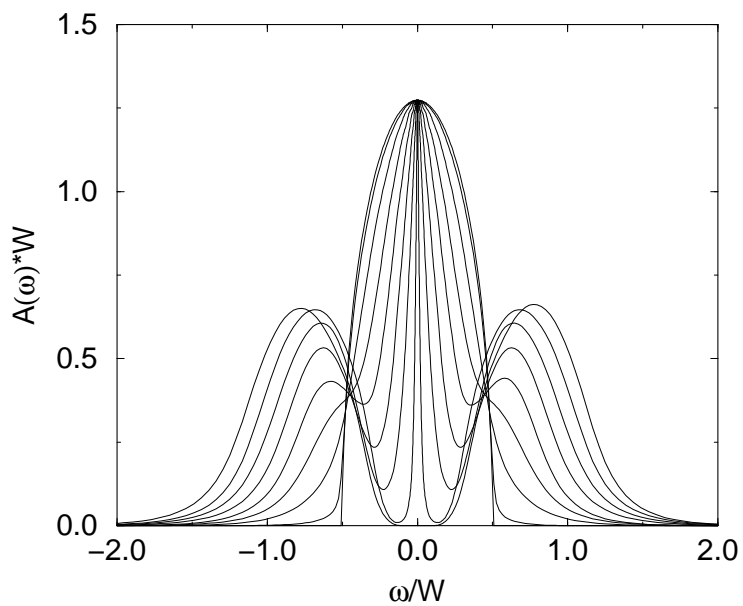

FIGURE 14. Evolution of the $T=0$ spectral function of the one-band Hubbard model with a semielliptic ("Bethe") DOS for interaction values $U / W=0,0.2,0.4, \ldots, 1.6$ ( $W$ : band width) calculated with the numerical renormalization group. At the critical interaction $U_{\mathrm{c} 2} / W \simeq 1.47$ the metallic solution disappears and the Mott gap opens; from Ref. [80].

increasing temperature. This is due to the fact that the insulator and the metal are not distinct phases in the crossover regime, implying that the insulator has a finite spectral weight at the Fermi level. This behavior has been detected experimentally by photoemission experiments [81].

Altogether, the thermodynamic transition line $U_{\mathrm{c}}(T)$ corresponding to the MottHubbard MIT is found to be of first order at finite temperatures, being associated with a hysteresis region in the interaction range $U_{\mathrm{c} 1}<U<U_{\mathrm{c} 2}$ where $U_{\mathrm{c} 1}$ and $U_{\mathrm{c} 2}$ are the values at which the insulating and metallic solution, respectively, vanishes [52, 58], [82], $[78,79,83],[53,80]$. The high precision, state-of-the-art MIT phase diagram by Blümer [78] is shown in Fig. 16. The hysteresis region terminates at a critical point. For higher temperatures the transition changes into a smooth crossover from a bad metal to a bad insulator.

It is interesting to note that the slope of the phase transition line is negative down to $T=0$, which implies that for constant interaction $U$ the metallic phase can be reached from the insulator by decreasing the temperature $T$, i.e., by cooling. This anomalous behavior (which corresponds to the Pomeranchuk effect [84] in ${ }^{3} \mathrm{He}$, if we associate solid ${ }^{3} \mathrm{He}$ with the insulator and liquid ${ }^{3} \mathrm{He}$ with the metal) can be easily understood from the Clausius-Clapeyron equation $d U / d T=\Delta S / \Delta D$. Here $\Delta S$ is the difference between the entropy in the metal and in the insulator, and $\Delta D$ is the difference between the number of doubly occupied sites in the two phases. Within the single-site DMFT there is no exchange coupling $J$ between the spins of the electrons in the insulator, since the scaling (19) implies $J \propto-t^{2} / U \propto 1 / d \rightarrow 0$ for $d \rightarrow \infty$. Hence the entropy of the macroscopically degenerate insulating state is $S_{\text {ins }}=k_{B} \ln 2$ per electron down to $T=0$. This is larger than the entropy $S_{\text {met }} \propto T$ per electron in the Landau Fermi-liquid describing the metal, i.e., 


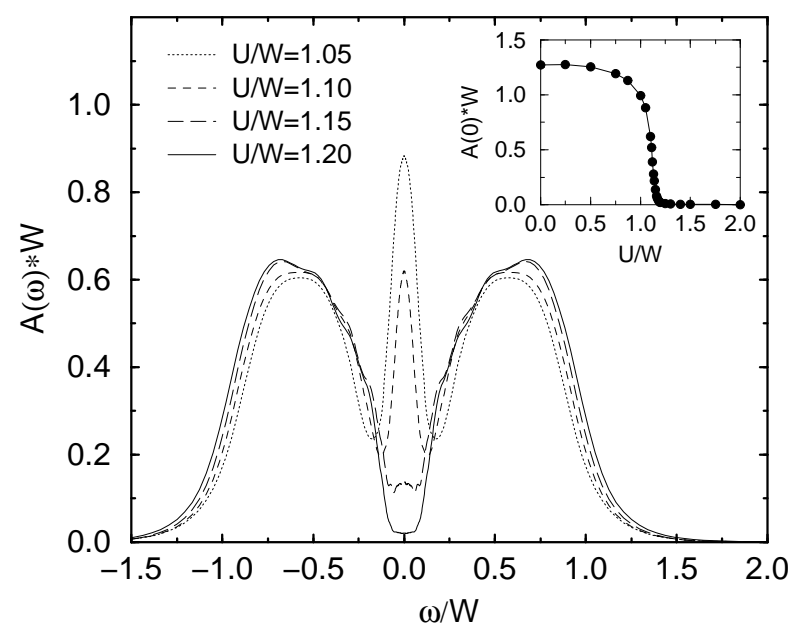

FIGURE 15. Spectral function for the half-filled Hubbard model for various values of $U$ at $T=$ $0.0276 \mathrm{~W}$ in the crossover region. The crossover from the metal to the insulator occurs via a gradual suppression of the quasiparticle peak at $\omega=0$. The inset shows the $U$ dependence of $A(\omega=0)$, in particular the rapid decrease for $U \approx 1.1 \mathrm{~W}$; from Ref. [79].

$\Delta S=S_{\text {met }}-S_{\text {ins }}<0$. At the same time the number of doubly occupied sites is lower in the insulator than in the metal, i.e., $\Delta D=D_{\text {met }}-D_{\text {ins }}>0$. The Clausius-Clapeyron equation then implies that the phase-transition line $T$ vs. $U$ has a negative slope down to $T=0$. However, this is an artifact of the single-site DMFT. Namely, there will always exist an exchange coupling between the electrons leading to a vanishing entropy of the insulator at $T=0$. Since the entropy of the insulator vanishes faster than linearly with the temperature, the difference $\Delta S=S_{\text {met }}-S_{\text {ins }}$ eventually becomes positive, whereby the slope also becomes positive at lower temperatures ${ }^{14}$; this is indeed observed in cluster DMFT calculations [85]. Since $\Delta S=0$ at $T=0$ the phase boundary must terminate at $T=0$ with infinite slope.

At half filling and for bipartite lattices in dimensions $d>2$ (in $d=2$ only at $T=0$ ), the paramagnetic phase is unstable against antiferromagnetic long-range order. The metalinsulator transition is then completely hidden by the antiferromagnetic insulating phase, as shown in Fig. 17.

\footnotetext{
${ }^{14}$ Here we assume for simplicity that the metal remains a Fermi liquid, and the insulator stays paramagnetic, down to the lowest temperatures. In fact, a Cooper pair instability will eventually occur in the metal, and the insulator will become long-range ordered, too. In this case the slope $d U / d T$ can change sign several times depending on the value of the entropy of the two phases across the phase transition.
} 


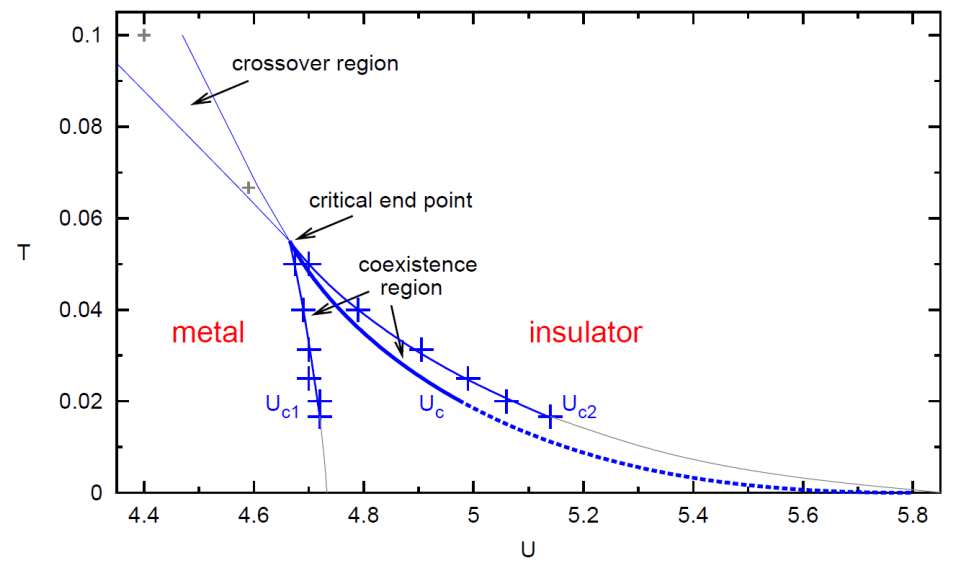

FIGURE 16. High precision Mott-Hubbard MIT phase diagram showing the metallic phase and the insulating phase, respectively, at temperatures below the critical end point, as well as a coexistence region; from Ref. [78].

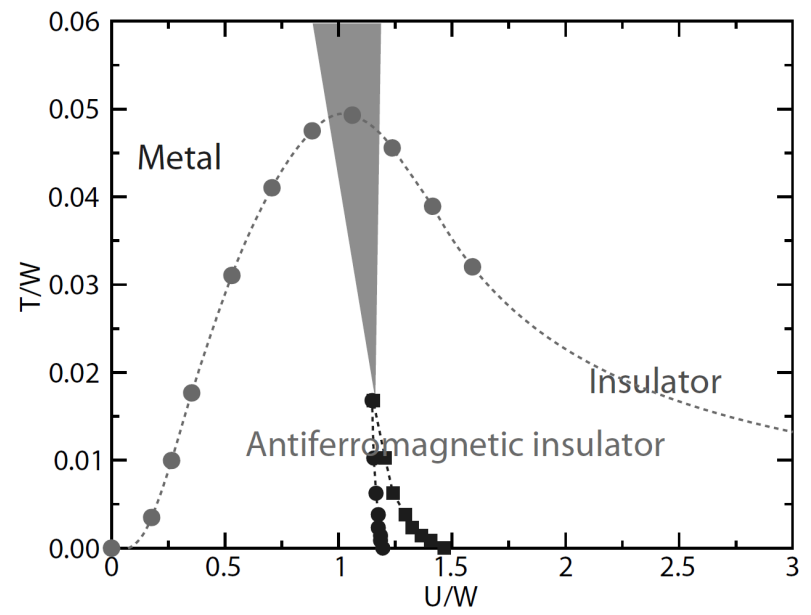

FIGURE 17. On bipartite lattices and for half filling $(n=1)$ the paramagnetic phase is unstable against antiferromagnetism. The metal-insulator transition is then completely hidden by the antiferromagnetic insulating phase; from Ref. [86].

\section{ELECTRONIC CORRELATIONS AND DISORDER}

The properties of real materials are strongly influenced by the electronic interaction and randomness. In particular, Coulomb correlations and disorder are both driving forces behind metal-insulator transitions (MITs) connected with the localization and delocalization of particles. While the Mott-Hubbard MIT is caused by the electronic repulsion 
$[5,68,87]$, the Anderson MIT is due to coherent backscattering of non-interacting particles from randomly distributed impurities [41, 88]. The interplay between disorder and interaction effects gives rise to many fascinating phenomena [88-93], some of which will now be discussed following the presentation of Refs. [94-96].

The Mott-Hubbard MIT is characterized by the opening of a gap in the density of states at the Fermi level. By contrast, at the Anderson localization transition the character of the spectrum at the Fermi level changes from a continuous spectrum to a dense point spectrum. Both MITs can be characterized by a single quantity, the local density of states (LDOS). Although the LDOS is not an order parameter associated with a symmetrybreaking phase transition, it discriminates between a metal and an insulator, which is driven by correlations and disorder.

\subsection{Arithmetic $v s$. geometric averaging over the disorder}

The theoretical investigation of disordered systems requires the use of probability distribution functions (PDFs) for the random quantities of interest. In physical or statistical problems one is usually interested in "typical" values of random quantities which are mathematically determined by the most probable value of the PDF, i.e., where the PDF becomes maximal. In many cases the complete PDF is not known, i.e., only limited information about the system provided by certain averages (moments or cumulants) is available. In this situation it is of great importance to choose the most informative average of a random variable. For example, if the PDF of a random variable has a single peak and fast decaying tails this variable is usually well estimated by its first moment, known as the arithmetic average. The arithmetic average of a function $F\left(\varepsilon_{i}\right)$ is defined by (see also (50))

$$
F_{\text {arith }} \equiv\left\langle F\left(\varepsilon_{i}\right)\right\rangle_{\mathrm{av}}=\int d \varepsilon_{i} P\left(\varepsilon_{i}\right) F\left(\varepsilon_{i}\right)
$$

However, there are many examples, e.g., from astronomy, the physics of glasses or networks, economy, sociology, biology or geology, where the knowledge of the arithmetic average is insufficient since the PDF is so broad that its characterization requires infinitely many moments. Such systems are said to be non-self-averaging. One example is Anderson localization: when a disordered system is near the Anderson MIT [41], most of the electronic quantities fluctuate strongly and the corresponding PDFs possess long tails [97-100]. At the Anderson MIT the corresponding moments might not even exist. This is well illustrated by the local density of states (LDOS) of the system. The arithmetic mean of this random one-particle quantity does not resemble its typical value at all. In particular, it is non-critical at the Anderson transition [101] and hence cannot help to detect the localization transition. In this case the geometric mean [102-104],

$$
F_{\text {geom }}=\exp \left[\left\langle\ln F\left(\varepsilon_{i}\right)\right\rangle_{\text {av }}\right]
$$

gives a much better approximation of the most probable ("typical") value of the LDOS. It vanishes at a critical strength of the disorder and hence provides an explicit criterion for Anderson localization [41, 105-107], [94-96]. 


\subsection{The Anderson-Hubbard model}

The fundamental electronic correlation model investigated here is the AndersonHubbard model

$$
\hat{H}=-t \sum_{i j, \sigma} \hat{c}_{i \sigma}^{+} \hat{c}_{j \sigma}+\sum_{i \sigma} \varepsilon_{i} n_{i \sigma}+U \sum_{i} \hat{n}_{i \uparrow} \hat{n}_{i \downarrow} .
$$

The ionic energy $\varepsilon_{i}$ is a random, independent variable which describes the local, quenched disorder affecting the motion of the electrons. The disorder part is modeled by a corresponding $\operatorname{PDF} P\left(\varepsilon_{i}\right)$. For $P\left(\varepsilon_{i}\right)=0$ the system is called pure. Here we use the continuous PDF

$$
P\left(\varepsilon_{i}\right)=\frac{\Theta\left(\frac{\Delta}{2}-\left|\varepsilon_{i}\right|\right)}{\Delta},
$$

with $\Theta$ as the step function. The parameter $\Delta$ is a measure of the disorder strength.

A non-perturbative theoretical framework for the investigation of correlated lattice electrons with a local interaction is again given by the DMFT. If in this approach the effect of local disorder is taken into account through the arithmetic mean of the LDOS [108] one obtains, in the absence of interactions, the well-known coherent potential approximation [46] (see Sec. 4.1.), which does not describe the physics of Anderson localization. To overcome this deficiency Dobrosavljević and collaborators formulated a variant of the DMFT where the geometrically averaged LDOS is computed from the solutions of the self-consistent stochastic DMFT equations [105] which is then incorporated into the self-consistency cycle [106]. Thereby a mean-field theory of Anderson localization can be derived which reproduces many of the expected features of the disorder-driven MIT for non-interacting electrons [106]. This scheme uses only one-particle quantities and is therefore easily incorporated into the DMFT for disordered electrons in the presence of phonons [109], or Coulomb correlations. In particular, the DMFT with geometrical averaging allows one to compute the phase diagram for the Anderson-Hubbard model with the continuous probability distribution function (119) at half filling [94]. In this way we find that, although the metallic phase is enhanced for small and intermediate values of the interaction and disorder, metallicity is eventually destroyed upon further increase of the disorder. Surprisingly, the Mott and Anderson insulators are found to be continuously connected. The phase diagram for the nonmagnetic ground state is shown in Fig. 18.

Interacting quantum many-particle systems with disorder pose fundamental challenges for theory and experiment not only in condensed matter physics [68, 87-91], but most recently also in the field of cold atoms in optical lattices [110-115]. Indeed, ultracold gases have quickly developed into a fascinating new laboratory for quantum many-body physics [110, 111, 116-120]. A major advantage of cold atoms in optical lattices is the high degree of controllability of the interaction and the disorder strength. In particular, these quantum many-body systems will allow for the first experimental investigation of the simultaneous presence of strong interactions and strong disorder. This very interesting parameter regime is not easily accessible in correlated electron materials. Namely, at or close to half filling where interaction effects become particularly pronounced, strong disorder implies fluctuations (e.g., of local energies) of the order of the band width, which usually leads to structural instabilities. These limitations are 


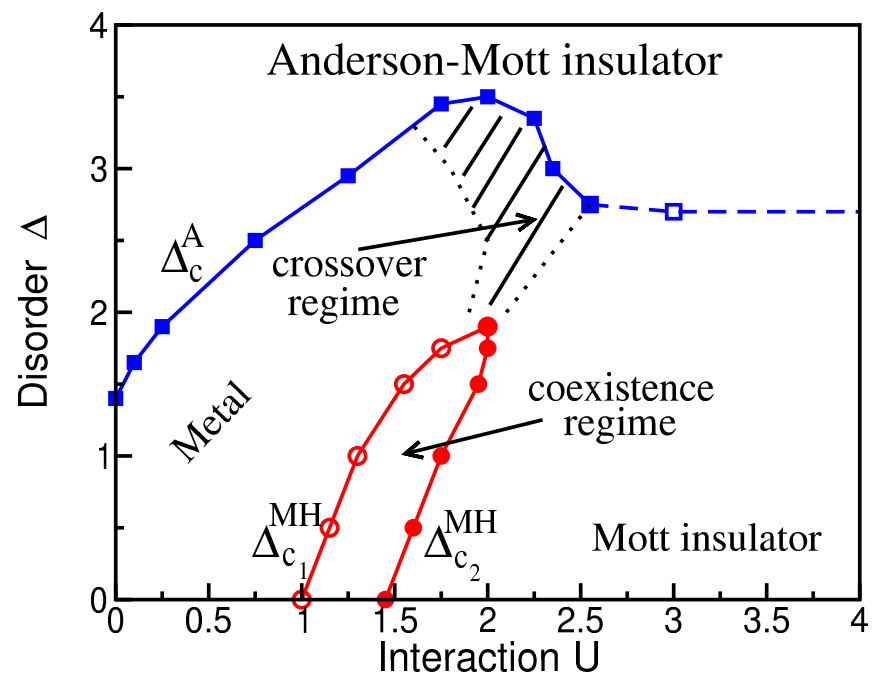

FIGURE 18. Non-magnetic ground-state phase diagram of the Anderson-Hubbard model at half filling as calculated by DMFT with the geometrically averaged ("typical") LDOS; after Ref. [94].

absent in the case of cold atoms in optical lattices where disorder can be tuned to become arbitrarily strong without destroying the experimental setup. Since at half filling and in the absence of frustration effects interacting fermions order antiferromagnetically, several basic questions arise:

(i) How is a non-interacting, Anderson localized system at half filling affected by a local interaction between the particles?

(ii) How does an antiferromagnetic insulator at half filling respond to disorder which in the absence of interactions would lead to an Anderson localized state?

(iii) Do Slater and Heisenberg antiferromagnets behave differently in the presence of disorder?

In Ref. [95] answers to the above questions were obtained by calculating the zero temperature, magnetic phase diagram of the disordered Hubbard model at half filling using DMFT with a geometric average over the disorder and allowing for a spin-dependence of the density of states (DOS). The results are collected in Fig. 19. Depending on whether the interaction $U$ is weak or strong the response of the system to disorder is found to be very different. At strong interactions, $U / W \gtrsim 1$, there exist only two phases, an AF insulating phase at weak disorder, $\Delta / W \lesssim 2.5$, and a paramagnetic Anderson-Mott insulator at strong disorder, $\Delta / W \gtrsim 2.5$. The transition between these two phases is continuous. By contrast, the non-magnetic phase diagram for weak interactions, $U / W \lesssim 1$, has a much richer structure (Fig. 18). In particular, for weak disorder a paramagnetic metallic phase is found to be stable. It is separated from the AF insulating phase at large $U$ by a narrow region of $A F$ metallic phase. The AF metallic phase is long-range ordered, but there is no gap since the disorder leads to a redistribution of spectral weight [95]. 


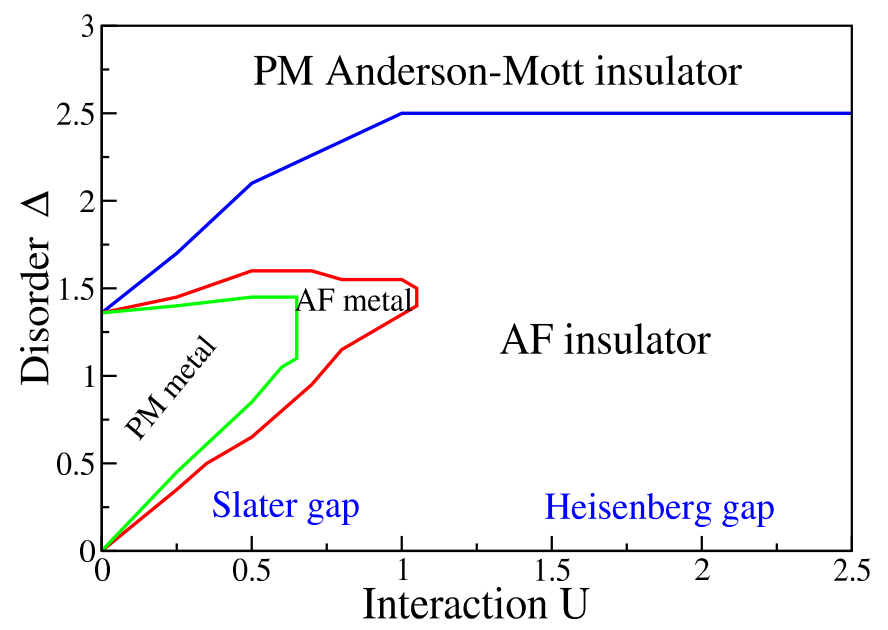

FIGURE 19. Magnetic ground-state phase diagram of the Anderson-Hubbard model at half filling as calculated by DMFT with the geometrically averaged ("typical”) LDOS; after Ref. [95].

\section{THEORY OF ELECTRONIC CORRELATIONS IN MATERIALS}

\subsection{The LDA+DMFT approach}

Although the Hubbard model is able to explain basic features of the phase diagram of correlated electrons it cannot explain the physics of real materials in any detail. Clearly, realistic theories must take into account the explicit electronic and lattice structure of the systems.

Until recently the electronic properties of solids were investigated by two essentially separate communities, one using model Hamiltonians in conjunction with many-body techniques, the other employing density functional theory (DFT) [121, 122]. DFT and its local density approximation (LDA) have the advantage of being ab initio approaches which do not require empirical parameters as input. Indeed, they are highly successful techniques for the calculation of the electronic structure of real materials [123]. However, in practice DFT/LDA is seriously restricted in its ability to describe strongly correlated materials where the on-site Coulomb interaction is comparable with the band width. Here, the model Hamiltonian approach is more general and powerful since there exist systematic theoretical techniques to investigate the many-electron problem with increasing accuracy. Nevertheless, the uncertainty in the choice of the model parameters and the technical complexity of the correlation problem itself prevent the model Hamiltonian approach from being a flexible or reliable enough tool for studying real materials. The two approaches are therefore complementary. In view of the individual power of DFT/LDA and the model Hamiltonian approach, respectively, it had always been clear that a combination of these techniques would be highly desirable for $a b$ initio investigations of real materials, including, e.g., $f$-electron systems and Mott insulators. One of the first successful attempts in this direction was the LDA+U method [124, 125], which 
combines LDA with a basically static, i.e., Hartree-Fock-like, mean-field approximation for a multi-band Anderson lattice model (with interacting and non-interacting orbitals). This method proved to be a very useful tool in the study of long-range ordered, insulating states of transition metals and rare-earth compounds. However, the paramagnetic metallic phase of correlated electron systems such as high-temperature superconductors and heavy-fermion systems clearly requires a treatment that goes beyond a static meanfield approximation and includes dynamical effects, e.g., the frequency dependence of the self-energy.

Here the recently developed LDA+DMFT method, a new computational scheme which merges electronic band structure calculations and the dynamical mean-field theory, has proved to be a breakthrough [126-131], [53, 132-135]. Starting from conventional band structure calculations in the local density approximation (LDA) the correlations are taken into account by the Hubbard interaction and a Hund's rule coupling term. The resulting DMFT equations are solved numerically with a quantum MonteCarlo (QMC) algorithm. By construction, LDA+DMFT includes the correct quasiparticle physics and the corresponding energetics. It also reproduces the LDA results in the limit of weak Coulomb interaction $U$. More importantly, LDA+DMFT correctly describes the correlation induced dynamics near a Mott-Hubbard MIT and beyond. Thus, LDA+DMFT is able to account for the physics at all values of the Coulomb interaction and doping level.

In the LDA+DMFT approach [126, 130, 131], [53] the LDA band structure is expressed by a one-particle Hamiltonian $\hat{H}_{\mathrm{LDA}}^{0}$, and is then supplemented by the local Coulomb repulsion $U$ and Hund's rule exchange $J$ (here we follow the presentation of Ref. [80]). This leads to a material specific generalization of the one-band model Hamiltonian

$$
\hat{H}=\hat{H}_{\mathrm{LDA}}^{0}+U \sum_{m} \sum_{i} \hat{n}_{i m \uparrow} \hat{n}_{i m \downarrow}+\sum_{i, m \neq m^{\prime}, \sigma, \sigma^{\prime}}\left(V-\delta_{\sigma \sigma^{\prime}} J\right) \hat{n}_{i m \sigma} \hat{n}_{i m^{\prime} \sigma^{\prime}}
$$

Here $m$ and $m^{\prime}$ enumerate the three interacting $t_{2 g}$ orbitals of the transition metal ion or the $4 f$ orbitals in the case of rare earth elements. The interaction parameters are related by $V=U-2 J$ which holds exactly for degenerate orbitals and is a good approximation for the $t_{2 g}$. The actual values for $U$ and $V$ can be obtained from an averaged Coulomb parameter $\bar{U}$ and Hund's exchange $J$, which can be calculated by constrained LDA.

In the one-particle part of the Hamiltonian

$$
\hat{H}_{\mathrm{LDA}}^{0}=\hat{H}_{\mathrm{LDA}}-\sum_{i} \sum_{m \sigma} \Delta \varepsilon_{d} \hat{n}_{i m \sigma}
$$

the energy term containing $\Delta \varepsilon_{d}$ is a shift of the one-particle potential of the interacting orbitals. It cancels the Coulomb contribution to the LDA results, and can also be calculated by constrained LDA [131].

Within the LDA+DMFT scheme the self-consistency condition connecting the selfenergy $\Sigma$ and the Green function $G$ at frequency $\omega$ reads:

$$
G_{q m, q^{\prime} m^{\prime}}(\omega)=\frac{1}{V_{B}} \int d^{3} k \quad\left(\left[\omega \mathbf{1}+\mu \mathbf{1}-H_{\mathrm{LDA}}^{0}(\boldsymbol{k})-\boldsymbol{\Sigma}(\omega)\right]^{-1}\right)_{q m, q^{\prime} m^{\prime}} .
$$


Here, 1 is the unit matrix, $\mu$ the chemical potential, $H_{\mathrm{LDA}}^{0}(\boldsymbol{k})$ is the orbital matrix of the LDA Hamiltonian derived, for example, in a linearized muffin-tin orbital (LMTO) basis, $\boldsymbol{\Sigma}(\omega)$ denotes the self-energy matrix which is nonzero only between the interacting orbitals, and $[\ldots]^{-1}$ implies the inversion of the matrix with elements $n(=q m), n^{\prime}\left(=q^{\prime} m^{\prime}\right)$, where $q$ and $m$ are the indices of the atom in the primitive cell and of the orbital, respectively. The integration extends over the Brillouin zone with volume $V_{B}$ (we note that $\hat{H}_{\mathrm{LDA}}^{0}$ may include additional non-interacting orbitals).

For cubic transition metal oxides Eq. (122) can be simplified to

$$
G(\omega)=G^{0}(\omega-\Sigma(\omega))=\int d \varepsilon \frac{N^{0}(\varepsilon)}{\omega-\Sigma(\omega)-\varepsilon}
$$

if the degenerate $t_{2 g}$ orbitals crossing the Fermi level are well separated from the other orbitals [131]. For non-cubic systems the degeneracy is lifted. In this case we employ Eq. (123) as an approximation, using different $\Sigma_{m}(\omega), N_{m}^{0}(\varepsilon)$ and $G_{m}(\omega)$ for the three non-degenerate $t_{2 g}$ orbitals.

The Hamiltonian (120) is solved within the DMFT using standard quantum MonteCarlo (QMC) techniques [36] to solve the self-consistency equations. From the imaginary time QMC Green function we calculate the physical (real frequency) spectral function with the maximum entropy method [136].

\subsection{Single-Particle Spectrum of Correlated Electrons in Materials}

Transition metal oxides are an ideal laboratory for the study of electronic correlations in solids. Among these materials, cubic perovskites have the simplest crystal structure and thus may be viewed as a starting point for understanding the electronic properties of more complex systems. Typically, the $3 d$ states in those materials form comparatively narrow bands with width $W \sim 2-3 \mathrm{eV}$, which leads to strong Coulomb correlations between the electrons. Particularly simple are transition metal oxides with a $3 d^{1}$ configuration since, among others, they do not show a complicated multiplet structure.

Photoemission spectra provide a direct experimental tool to study the electronic structure and spectral properties of electronically correlated materials. Intensive experimental investigations of spectral and transport properties of strongly correlated $3 d^{1}$ transition metal oxides started with investigations by Fujimori et al. [137]. These authors observed a pronounced lower Hubbard band in the photoemission spectra (PES) which cannot be explained by conventional band structure theory.

\subsubsection{Excursion: Detection of electronic correlations in solids by photoemission spectroscopy}

In photoemission spectroscopy (PES) a photon of a given energy is used to emit an electron whose properties (energy, angular distribution) are measured in a detector. Angular resolved PES is referred to as ARPES. This technique measures occupied electronic states. This means that only the states described by the full spectral function 

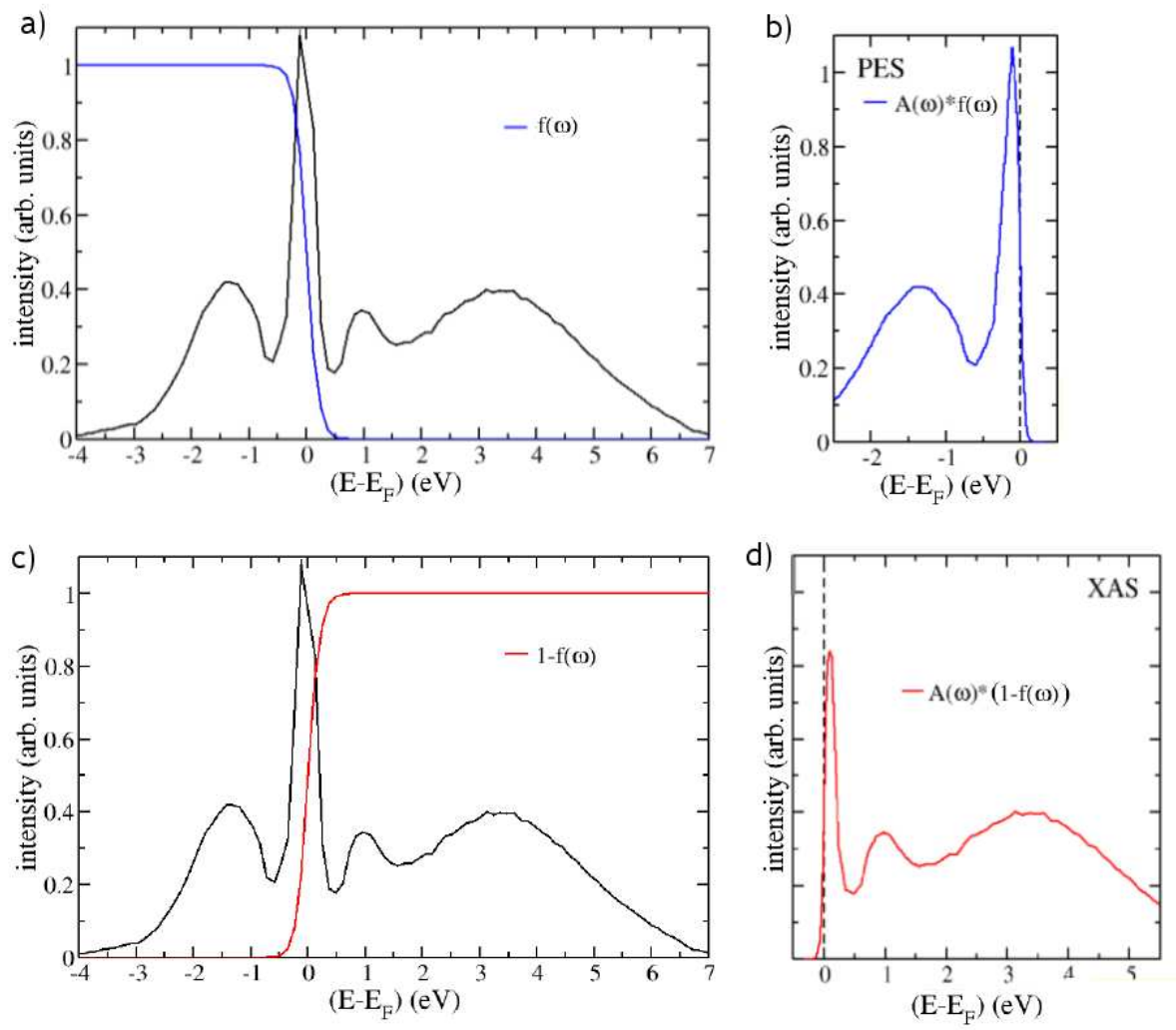

FIGURE 20. Using photoemission spectroscopy (PES) the occupied electronic states can be measured. This corresponds to a multiplication of the (unknown) spectral function of a material with the Fermi function $f(\omega)$ as shown in panel a). PES thus only measures the lower part of the full spectral function as shown in panel b). Inverse photoemission spectroscopy (IPES) or techniques like x-ray absorption spectroscopy (XAS) measure the unoccupied states. This corresponds to a multiplication of the spectral function with $1-f(\omega)$ as shown in panel c). Hence IPES or XAS measures the upper part of the full spectral function as shown in panel d).

of a material multiplied by the Fermi function $f(\omega)$ are measured (see Fig. 20a), leading to the typical result shown in Fig. 20b. By contrast, inverse photoemission spectroscopy (IPES) measures the unoccupied electronic states. IPES is harder to perform and not as accurate as PES. But in many situations information about the unoccupied states is also available by X-ray absorption spectroscopy (XAS). In this case only the states described by the spectral function of a material multiplied by $1-f(\omega)$ (Fig. 20c) are measured, leading to a typical result shown in Fig. 20d.

Spectroscopic techniques are routinely used to investigate correlated electronic systems. They provide very valuable information about the system since they measure the spectral function of a material which can be calculated theoretically. In particular, pho- 
toemission techniques allow one to detect the correlation induced shift of spectral weight discussed in Sec. 5.

In the following we will employ the LDA+DMFT approach to compute the $\boldsymbol{k}$ integrated electronic spectra of two correlated materials, the $3 d^{1}$ system $(\mathrm{Sr}, \mathrm{Ca}) \mathrm{VO}_{3}$ and the charge-transfer insulator $\mathrm{NiO}$.

\subsection{2. $\mathrm{Sr}_{x} \mathrm{Ca}_{1-x} \mathrm{VO}_{3}$}

$\mathrm{SrVO}_{3}$ and $\mathrm{CaVO}_{3}$ are simple transition metal compounds with a $3 d^{1}$ configuration (here we follow the presentation of Sekiyama et al. [138] and Nekrasov et al. [139]). The main effect of the substitution of $\mathrm{Sr}$ ions by the isovalent, but smaller, $\mathrm{Ca}$ ions is to decrease the $\mathrm{V}-\mathrm{O}-\mathrm{V}$ angle from $\theta=180^{\circ}$ in $\mathrm{SrVO}_{3}$ to $\theta \approx 162^{\circ}$ in the orthorhombically distorted structure of $\mathrm{CaVO}_{3}$. However, this rather strong bond bending results only in a $4 \%$ decrease of the one-particle bandwidth $W$ and thus in a correspondingly small increase of the ratio $U / W$ as one moves from $\mathrm{SrVO}_{3}$ to $\mathrm{CaVO}_{3}$.

LDA+DMFT(QMC) spectra of $\mathrm{SrVO}_{3}$ and $\mathrm{CaVO}_{3}$ were calculated by Sekiyama et al. [138] by starting from the respective LDA DOS of the two materials; they are shown in Fig. 21. These spectra show genuine correlation effects, i.e., the formation of lower Hubbard bands at about $1.5 \mathrm{eV}$ and upper Hubbard bands at about $2.5 \mathrm{eV}$, with wellpronounced quasiparticle peaks at the Fermi energy. Therefore both $\mathrm{SrVO}_{3}$ and $\mathrm{CaVO}_{3}$ are strongly correlated metals. The DOS of the two systems shown in Fig. 21 are quite similar. In fact, $\mathrm{SrVO}_{3}$ is slightly less correlated than $\mathrm{CaVO}_{3}$, in accord with their different LDA bandwidths. The inset of Fig. 21 shows that the effect of temperature on the spectrum is small for $T \lesssim 700 \mathrm{~K}$. Spectra of $\mathrm{SrVO}_{3}$ and $\mathrm{CaVO}_{3}$ were also calculated independently by Pavarini et al.[140].

Since the three $t_{2 g}$ orbitals of this simple $3 d^{1}$ material are (almost) degenerate the spectral function has the same three-peak structure as that of the one-band Hubbard model shown in Fig. 15. The temperature induced decrease of the quasiparticle peak height is also clearly seen. As noted in Sec. 5 the actual form of the spectrum no longer resembles the input (LDA) DOS, i.e., it essentially depends only on the first three energy moments of the LDA DOS (electron density, average energy, band width). In the left panel of Fig. 22 the LDA+DMFT(QMC) spectra at $300 \mathrm{~K}$ are compared with experimental high-resolution bulk PES. For this purpose the theoretical spectra were multiplied with the Fermi function at the experimental temperature $(20 \mathrm{~K})$ and Gauss broadened with the experimental resolution of $0.1 \mathrm{eV}$ [138]. The quasiparticle peaks in theory and experiment are seen to be in very good agreement. In particular, their height and width are almost identical for both $\mathrm{SrVO}_{3}$ and $\mathrm{CaVO}_{3}$. The difference in the positions of the lower Hubbard bands may be partly due to (i) the subtraction of the (estimated) oxygen contribution which might also remove some $3 d$ spectral weight below $-2 \mathrm{eV}$, and (ii) uncertainties in the $a b$ initio calculation of the local Coulomb interaction strength. In the right panel of Fig. 22 comparison is made with XAS data of Inoue et al. [141]. Core-hole life time effects were considered by Lorentz broadening the spectrum with $0.2 \mathrm{eV}$ [142], multiplying with the inverse Fermi function (80K), and then Gauss broadening with the experimental resolution of $0.36 \mathrm{eV}$ [143]. Again, the overall 


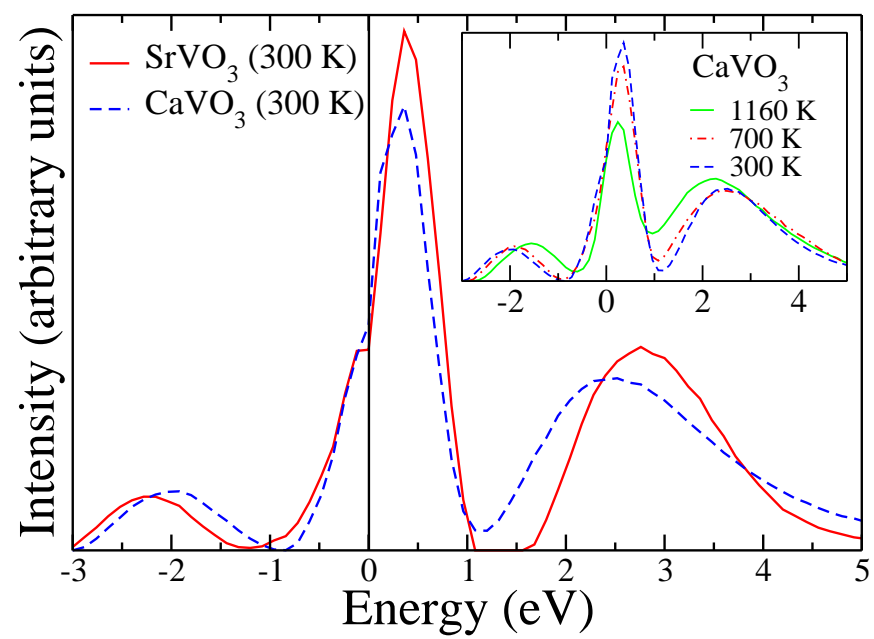

FIGURE 21. LDA+DMFT(QMC) spectrum of $\mathrm{SrVO}_{3}$ (solid line) and $\mathrm{CaVO}_{3}$ (dashed line) calculated at $\mathrm{T}=300 \mathrm{~K}$; inset: effect of temperature in the case of $\mathrm{CaVO}_{3}$; after Ref. [138].

agreement of the weights and positions of the quasiparticle and upper $t_{2 g}$ Hubbard band is good, including the tendencies when going from $\mathrm{SrVO}_{3}$ to $\mathrm{CaVO}_{3}\left(\mathrm{Ca}_{0.9} \mathrm{Sr}_{0.1} \mathrm{VO}_{3}\right.$ in the experiment). For $\mathrm{CaVO}_{3}$ the weight of the quasiparticle peak is somewhat lower than in the experiment. In contrast to one-band Hubbard model calculations, the material specific results reproduce the strong asymmetry around the Fermi energy w.r.t. weights and bandwidths. The results also give a different interpretation of the XAS than in Ref. [141] where the maximum at about $2.5 \mathrm{eV}$ was attributed to an $e_{g}$ band and not to the $t_{2 g}$ upper Hubbard band. The slight differences in the quasiparticle peaks (see Fig. 21) lead to different effective masses, namely $m^{*} / m=2.1$ for $\mathrm{SrVO}_{3}$ and $m^{*} / m=2.4$ for $\mathrm{CaVO}_{3}$. These theoretical values agree with $m^{*} / m=2-3$ for $\mathrm{SrVO}_{3}$ and $\mathrm{CaVO}_{3}$ as obtained from de Haas-van Alphen experiments and thermodynamics [144].

The experimentally determined spectra of $\mathrm{SrVO}_{3}$ and $\mathrm{CaVO}_{3}$ and the good agreement with parameter-free LDA+DMFT calculations confirm the existence of a pronounced three-peak structure in a correlated bulk material. Although the DMFT had predicted such a behavior for the Hubbard model (see Sec. 5.1.) it was not clear whether the DMFT result would really be able to describe real materials in three dimensions. Now it has been confirmed that the three-peak structure not only occurs in single-impurity Anderson models but also in three-dimensional correlated bulk matter.

\subsection{3. $\mathrm{NiO}$}

Already in 1937, at the outset of modern solid state physics, de Boer and Verwey [145] drew attention to the surprising properties of materials with incompletely filled $3 d$-bands, such as NiO. This observation prompted Peierls and Mott [146] to discuss 


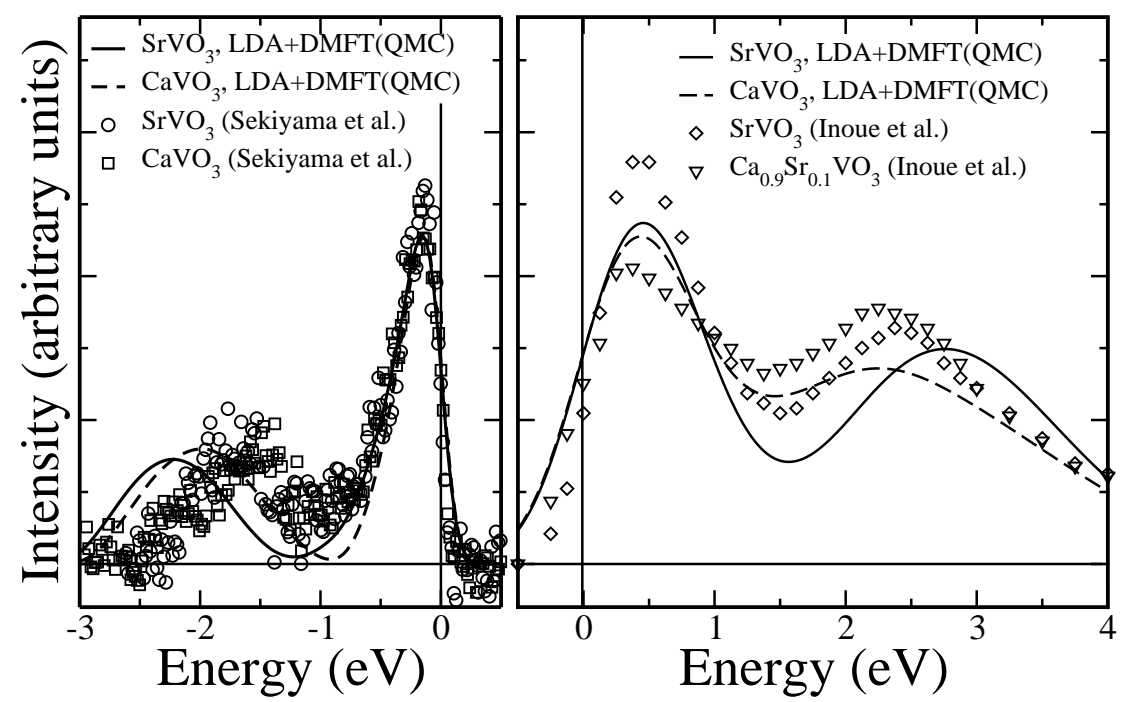

FIGURE 22. Comparison of the calculated, parameter-free LDA+DMFT(QMC) spectra of $\mathrm{SrVO}_{3}$ (solid line) and $\mathrm{CaVO}_{3}$ (dashed line) with experiment. Left: Bulk-sensitive high-resolution $\mathrm{PES}\left(\mathrm{SrVO}_{3}\right.$ : circles; $\mathrm{CaVO}_{3}$ : rectangles). Right: $1 \mathrm{~s} \mathrm{XAS}$ for $\mathrm{SrVO}_{3}$ (diamonds) and $\mathrm{Ca}_{0.9} \mathrm{Sr}_{0.1} \mathrm{VO}_{3}$ (triangles) [141]. Horizontal line: experimental subtraction of the background intensity; after Ref. [139].

the interaction between the electrons. Ever since transition metal oxides (TMOs) were investigated intensively (here we follow the presentation of Kuneš et al.[147]). This interest further increased when it was discovered that TMOs display an amazing multitude of ordering and electron correlation phenomena, including high temperature superconductivity, colossal magnetoresistance and Mott metal-insulator transitions [1]. In the late 1950 's $\mathrm{MnO}$ and $\mathrm{NiO}$ were taken as the textbook examples of antiferromagnets. However, when the importance of local Coulomb correlations in the transition metal $d$-shell was realized TMOs were considered candidates for Mott insulators [146]. In the mid 1980's Zaanen, Sawatzky and Allen (ZSA) introduced their classification of TMOs and related compounds into Mott-Hubbard and charge-transfer (CT) systems [148]. In the early TMOs the ligand $p$-band is located well below the transition metal $d$-band and thus plays a minor role in the low energy dynamics. Such a case, called Mott-Hubbard system in the ZSA scheme, is well described by a multi-band Hubbard model. On the other hand, the late TMOs belong to the CT type where the $p$-band is situated between the interaction split $d$-bands. A more general Hamiltonian where the $p$-states are explicitly included is then needed, which can be viewed as a combination of multi-band Hubbard and Anderson lattice models. A major impulse for detailed investigations of CT systems, and especially of their hole doped regime, came with the discovery of high temperature superconductivity in cuprate perovskites. While the standard three-band Hamiltonian for cuprates [149] contains only one $d$-orbital per lattice site, the description of cubic 
transition metal monoxides, the prominent member of which is $\mathrm{NiO}$, requires the full set of $d$-orbitals. The latter are of interest not only for fundamental research, but play an important role also in fields such as geophysics [150]. Furthermore, recent progress in high pressure experiments [151] made the insulator-to-metal transition in some TMOs accessible in the laboratory, providing yet another stimulus for theoretical investigations.

$\mathrm{NiO}$ is a type II antiferromagnet $\left(T_{N}=523 \mathrm{~K}\right)$ with a magnetic moment of almost $2 \mu_{B}$ and a large gap surviving well above $T_{N}$. The standard LDA band theory predicts $\mathrm{NiO}$ to be a metal [152], or an antiferromagnetic insulator [153] if spin polarization is allowed. A severe underestimation of the gap and the magnetic moment suggests, however, that the Slater antiferromagnetic state obtained within LDA does not describe the true nature of $\mathrm{NiO}$. On the other hand exact diagonalization studies on small clusters were quite successful in describing the single- and two-particle spectra [154], showing that the local Coulomb interactions are important. This made it clear that an explicit treatment of Coulomb interactions within the $3 d$ shell is needed, and methods such as $\mathrm{LDA}+\mathrm{U}$ [155], self-interaction correction [156], or GW [157] were applied. The static, orbitally dependent self-energy of LDA+U enforces a separation of the occupied and unoccupied $d$-bands and thus opens a gap comparable to experiment. This in turn leads to a significant improvement of the description of static properties such as the local moment or the lattice dynamics [158]. However, the LDA+U method is limited to an ordered state and does not yield the electronic excitations and the effect of doping correctly. A systematic inclusion of dynamical correlations is only made possible by the DMFT.

In Fig. 23 the calculated spectral densities [147] (resolved into Ni $3 d$ and O $2 p$ contributions) are compared to photoemission and inverse photoemission data [159]. Using the full $p-d$ Hamiltonian it is possible to cover the entire valence and conduction bands spectra. Features corresponding to $4 s$ and $4 p$ bands at $10 \mathrm{eV}$ and $13 \mathrm{eV}$, respectively, are not included in the theoretical spectrum. The relative intensity of the $2 p$ contribution increases with decreasing photon energy [160]. Therefore the $120 \mathrm{eV}$ spectrum is dominated by $\mathrm{Ni} 3 d$ emission, while at $66 \mathrm{eV}$ photon energy the $\mathrm{O} 2 p$ contribution peaked around $-4 \mathrm{eV}$ is resolved (for a detailed orbital decomposition see Ref. [160]). The theoretical spectrum very well reproduces the experimental features, including the size of the gap, the $d$ character of the conduction band, the broad $d$ peak at $-9 \mathrm{eV}$, the position of the $p$-band, and the strong $d$ contribution at the top of the valence band. While the gap and the Hubbard subbands can be described already with the static theory (LDA+U) [155], a dynamical treatment is apparently needed to capture the substantial redistribution of spectral weight between the incoherent $(-9 \mathrm{eV})$ and resonant $(-2 \mathrm{eV})$ features in the $d$ spectrum.

We see that by including the ligand $p$ states and the on-site Coulomb interaction within the same framework one is able to provide a full description of the valence band spectrum and, in particular, of the distribution of spectral weight between the lower Hubbard band and the resonant peak at the top of the valence band. Good agreement with the available photoemission and inverse-photoemission data is found without need for adjustable parameters [147]. In a similar way ARPES data can also be explained in detail [161]. 


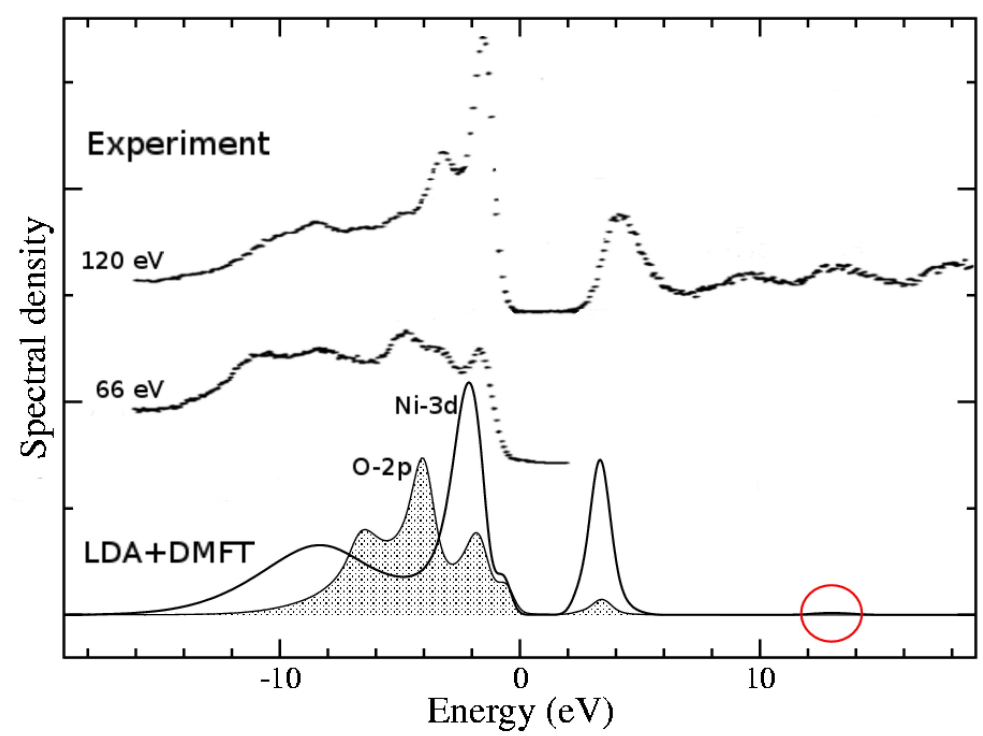

FIGURE 23. Theoretical Ni- $d$ (solid line) and O- $p$ (shaded) resolved spectral densities compared to photoemission and inverse photoemission data obtained at $120 \mathrm{eV}$ and $66 \mathrm{eV}$ photon frequencies after Ref. [159]. Gaussian broadening of $0.6 \mathrm{eV}$ full width at half maximum corresponding to the experimental resolution was applied to the theoretical curves. The circle marks position of the $d^{10} \underline{L}$ excitation; from Ref. [147].

\subsection{Correlation induced structural transformations}

In materials with correlated electrons the interaction between spin, charge, orbital, and lattice degrees of freedom leads to a wealth of ordering phenomena and complex phases [1]. The diverse properties of such systems and their great sensitivity with respect to changes of external parameters such as temperature, pressure, magnetic field or doping also make them highly attractive for technological applications [1]. In particular, orbital degeneracy is an important and often inevitable cause for this complexity [162]. A fascinating example is the cooperative Jahn-Teller (JT) effect - the spontaneous lifting of the degeneracy of an orbital state - leading to an occupation of particular orbitals ("orbital ordering") and, simultaneously, to a structural relaxation with symmetry reduction.

Applications of LDA+DMFT so far mainly employed linearized and higher order muffin-tin orbital (L(N)MTO) methods [163] and concentrated on the study of correlation effects within the electronic system for a given ionic lattice (here we follow the presentation of Leonov et al. $[164,165])$. On the other hand, the interaction of the electrons with the ions also affects the lattice structure. LDA+DMFT investigations of particularly drastic examples, e.g., the volume collapse in paramagnetic $\mathrm{Ce}[166,167]$ and $\mathrm{Pu}$ [168-170], and the magnetic moment collapse in $\mathrm{MnO}$ [171], incorporated the lattice by calculating the total energy of the correlated material as a function of the atomic volume. However, for investigations going beyond equilibrium-volume calculations, e.g., of the 


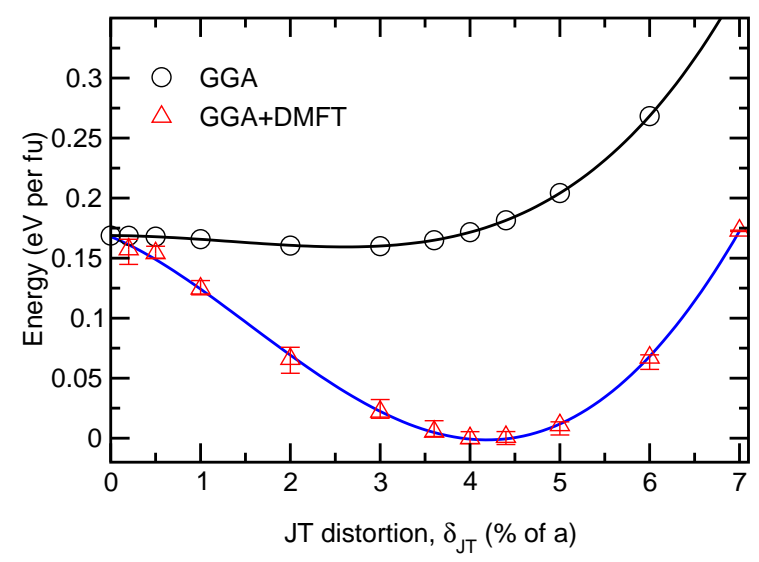

FIGURE 24. Comparison of the total energies of paramagnetic $\mathrm{KCuF}_{3}$ computed by GGA and GGA+DMFT(QMC) as a function of the JT distortion. Error bars indicate the statistical error of the DMFT(QMC) calculations; from Ref. [164].

cooperative JT effect and other subtle structural relaxation effects, the L(N)MTO method is not suitable since it cannot determine atomic displacements reliably. This is partly due to the fact that the atomic-sphere approximation used in the L(N)MTO scheme, with a spherical potential inside the atomic sphere, completely neglects multipole contributions to the electrostatic energy originating from the distorted charge density distribution around the atoms.

Recently Leonov et al. [164, 165] formulated a computational scheme which now allows one to calculate lattice-relaxation effects caused by electronic correlations. To this end the GGA+DMFT - a merger of the "generalized gradient approximation" (GGA) and DMFT — was formulated within a plane-wave pseudopotential approach $[172,173]$. Thereby the limitations of the L(N)MTO scheme in the direct calculation of total energies are overcome. In particular, this new method can be applied to determine the orbital order and the cooperative JT distortion in the paramagnetic phase of the prototypical JT system $\mathrm{KCuF}_{3}$.

$\mathrm{KCuF}_{3}$ is long known to be a prototypical material with a cooperative JT distortion [162] where the electronic degrees of freedom are the driving force behind the orbital order $[162,174,175]$. Indeed, the relatively high (tetragonal) symmetry makes $\mathrm{KCuF}_{3}$ one of the simplest systems to study. In particular, only a single internal structure parameter, the shift of the in-plane fluorine atom from the $\mathrm{Cu}-\mathrm{Cu}$ bond center, is needed to describe the lattice distortion.

The total energies as a function of the JT distortion $\delta_{J T}=\frac{1}{2}\left(d_{l}-d_{s}\right) /\left(d_{l}+d_{s}\right)$, where $d_{l}$ and $d_{s}$ denote the long and short $\mathrm{Cu}-\mathrm{F}$ bond distances, obtained by the GGA and GGA+DMFT, respectively, are compared in Fig. 24. It should be noted that the GGA not only predicts a metallic solution, but its total energy is seen to be almost constant for $0<\delta_{J T} \lesssim 4 \%$. Both features are in contradiction to experiment since the extremely shallow minimum at $\delta_{J T} \simeq 2.5 \%$ would imply that $\mathrm{KCuF}_{3}$ has no JT distortion for $T \gtrsim$ $100 \mathrm{~K}$. By contrast, the inclusion of the electronic correlations among the partially filled 
$\mathrm{Cu} e_{g}$ states in the GGA+DMFT approach leads to a very substantial lowering of the total energy by $\sim 175 \mathrm{meV}$ per formula unit (fu). This implies that the strong JT distortion persists up to the melting temperature $(>1000 \mathrm{~K})$, in agreement with experiment. The minimum of the GGA+DMFT total energy is located at the value $\delta_{J T}=4.2 \%$ which is also in excellent agreement with the experimental value of $4.4 \%$ [176]. This clearly shows that the $\mathrm{JT}$ distortion in paramagnetic $\mathrm{KCuF}_{3}$ is caused by electronic correlations.

The GGA+DMFT scheme introduced in Refs. [164, 165] opens the way for fully microscopic investigations of the structural properties of strongly correlated electron materials such as lattice instabilities observed at correlation induced metal-insulator transitions.

\section{KINKS IN THE DISPERSION OF STRONGLY CORRELATED ELECTRON SYSTEMS}

The dispersion relation $E_{k}$ indicates at which energy and crystal momentum one-particle excitations can occur in a solid. The coupling between the excitations may lead to abrupt changes in the slope of the dispersion, referred to as "kinks". Such kinks thus carry important information about interactions in a many-body system (here we follow the presentation of Byczuk et al. [188, 189]).

In systems with a strong electron-phonon coupling kinks in the electronic dispersion at 40-60 meV below the Fermi level are well known ("Kohn anomaly"). Therefore the kinks detected at 40-70 meV below the Fermi level in the electronic dispersion of high-temperature superconductors are taken as evidence for phonon [177, 178] or spinfluctuation based $[179,180]$ pairing mechanisms. Kinks in the electronic dispersion at binding energies ranging from 30 to $800 \mathrm{meV}$ are also found in various other metals [181, 182, 185-187] posing questions about their origins.

Starting from the unexpected finding of kinks in the momentum-resolved spectral functions of $\mathrm{SrVO}_{3}$ calculated by LDA+DMFT [190], Byczuk et al. [188] recently discovered a novel, purely electronic mechanism yielding kinks in the electron dispersion. This mechanism does not require a coupling of two different excitations as in previously known cases. The theory applies to strongly correlated metals whose spectral function shows well separated Hubbard subbands and central peak as, for example, in transition metal-oxides. For a microscopic description of these electronic kinks the single-band Hubbard model (4) with particle-hole symmetry was investigated by the DMFT at $T=0$ [188] as will be described below.

The effective dispersion relation $E_{k}$ of the one-particle excitation is determined by the singularities of $G(\boldsymbol{k}, \omega)=\left(\omega+\mu-\varepsilon_{\mathbf{k}}-\Sigma(\boldsymbol{k}, \omega)\right)^{-1}$, which give rise to peaks in the spectral function $A(\boldsymbol{k}, \omega)=-\operatorname{Im} G(\boldsymbol{k}, \omega) / \pi$. Here $\omega$ is the frequency, $\mu$ the chemical potential, $\varepsilon_{k}$ the bare dispersion relation, and $\Sigma(k, \omega)$ is the self-energy. If the damping given by the imaginary part of $\Sigma(k, \omega)$ is not too large, the effective dispersion $E_{k}$ is thus determined by

$$
E_{k}+\mu-\varepsilon_{k}-\operatorname{Re} \Sigma\left(k, E_{k}\right)=0 .
$$

Any kinks in $E_{k}$ that do not originate from $\varepsilon_{k}$ must therefore be due to changes in the slope of $\operatorname{Re} \Sigma(\boldsymbol{k}, \omega)$. 

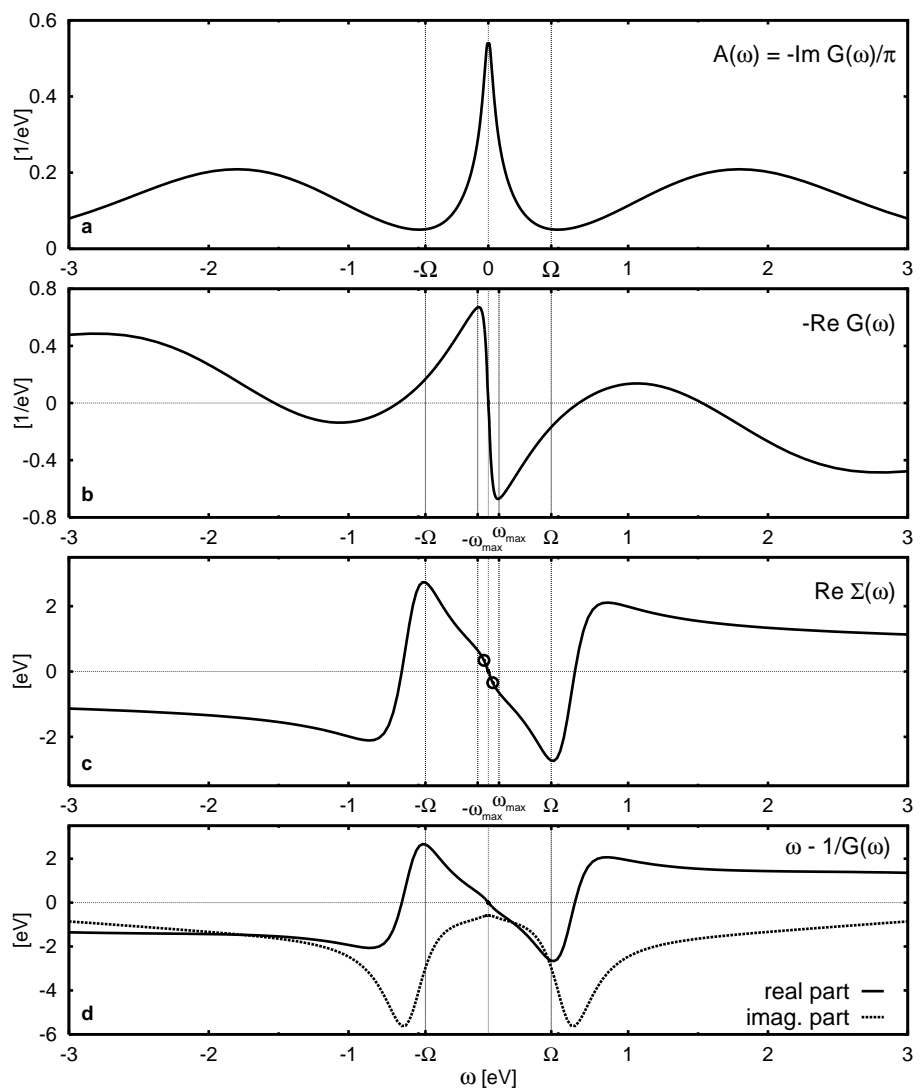

FIGURE 25. Local propagator and self-energy for a strongly correlated system. (a) Correlation-induced three-peak spectral function $A(\omega)=-\operatorname{Im} G(\omega) / \pi$ with dips at $\pm \Omega=0.45 \mathrm{eV}$. (b) Corresponding real part of the propagator, $-\operatorname{Re} G(\omega)$, with minimum and maximum at $\pm \omega_{\max }$ inside the central spectral peak. (c) Real part of the self-energy with kinks at $\pm \omega_{*}$ (circles), located at the points of maximum curvature of $\operatorname{Re} G(\omega),\left(\omega_{*}=0.4 \omega_{\max }=0.03 \mathrm{eV}\right)$. (d) $\omega-1 / G(\omega)$ contributes to the self-energy. In general $\operatorname{Re}[\omega-1 / G(\omega)]$ (full line) is linear in $|\omega|<\Omega$. The other contribution to the self-energy is $-\Delta(G(\omega)) \approx-\left(m_{2}-m_{1}^{2}\right) G(\omega)$ (to lowest order in the moments $m_{i}$ of $\varepsilon_{k}$; here $m_{2}-m_{1}^{2}=0.5 \mathrm{eV}^{2}$ ). Therefore the nonlinearity of $-\operatorname{Re}[G(\omega)]$ at $\pm \omega_{*}$ determines the location of kinks; after Ref. [188].

The DMFT self-consistency equations are now used to express $\Sigma(\boldsymbol{k}, \omega)=\Sigma(\omega)$ as $\Sigma(\omega)=\omega+\mu-1 / G(\omega)-\Delta(G(\omega))$, where $G(\omega)=\int G(\boldsymbol{k}, \omega) d \boldsymbol{k}$ is the $\boldsymbol{k}$-averaged local Green function and $\Delta(G)$ is an energy-dependent hybridization function, written here as a function of $G(\omega)$.

Kinks in $\operatorname{Re} \Sigma(\omega)$ are found to appear at a new small energy scale which emerges quite generally for a three-peak spectral function $A(\omega)$, see Fig. 25. Kramers-Kronig relations imply that $\operatorname{Re}[G(\omega)]$ is small near the dips of $A(\omega)$, located at $\pm \Omega$. Therefore $\operatorname{Re}[G(\omega)]$ has a maximum and a minimum at $\pm \omega_{\max }$ inside the central spectral peak 
(Fig. 25b). This directly leads to kinks in $\operatorname{Re} \Sigma(\omega)$ for the following reason. There are two contributions to $\Sigma(\omega): \omega+\mu-1 / G(\omega)$ and $-\Delta(G(\omega))$. While $\operatorname{Re}[\omega+\mu-1 / G(\omega)]$ is linear in the large energy window $|\omega|<\Omega$ (Fig. 25d), the term $-\operatorname{Re}[\Delta(G(\omega))]$ is approximately proportional to $-\operatorname{Re}[G(\omega)]$ (at least to first order in a moment expansion), and thus remains linear only in a much narrower energy window $|\omega|<\omega_{\max }$. The sum of these two contributions produces pronounced kinks in the real part of the selfenergy at $\pm \omega_{*}$, where $\omega_{*}=0.41 \omega_{\max }$ is the energy where $\operatorname{Re}[G(\omega)]$ has maximum curvature (marked by circles in Fig. 25c). The Fermi-liquid (FL) regime with slope $\partial \operatorname{Re} \Sigma(\omega) / \partial \omega=1-1 / Z_{\mathrm{FL}}$ thus extends only throughout a small part of the central peak $\left(|\omega|<\omega_{\star}\right)$. At intermediate energies $\left(\omega_{\star}<|\omega|<\Omega\right)$ the slope is then given by $\partial \operatorname{Re} \Sigma(\omega) / \partial \omega=1-1 / Z_{\mathrm{CP}}$. The kinks at $\pm \omega_{*}$ mark the crossover between these two slopes. As a consequence there is also a kink at $\omega_{*}$ in the effective band structure $E_{k}$ (Fig. 26).

The FL regime terminates at the kink energy scale $\omega_{\star}$, which cannot be determined within FL theory itself. One of the most surprising results of the investigation is the fact that it is possible to describe properties of the system outside the Landau-Fermi-liquid regime fully analytically. Namely, one can express the quantities $\omega_{\star}$ and $Z_{\boldsymbol{C P}}$ in terms of $Z_{\mathrm{FL}}$ and the bare density of states alone. Explicitly, one finds $\omega_{\star}=0.41 Z_{\mathrm{FL}} D$, where $D$ is an energy scale of the noninteracting system, e.g., $D$ is approximately given by half the bandwidth [188].

The energy scale $\omega_{*}$ involves only the bare band structure which can be obtained, for example, from band structure calculations, and the FL renormalization $Z_{\mathrm{FL}}=1 /(1-$ $\partial \operatorname{Re} \Sigma(0) / \partial \omega) \equiv m / m^{*}$ known from, e.g., specific heat measurements or many-body calculations. It should be noted that since phonons are not involved in this mechanism, $\omega_{\star}$ shows no isotope effect. For strongly interacting systems, in particular close to a metal-insulator transition, $\omega_{\star}$ can become quite small, e.g., smaller than the Debye energy.

The theory described above explains the kinks in the slope of the dispersion as a direct consequence of the electronic interaction [188]. The same mechanism may also lead to kinks in the low-temperature electronic specific heat [191]. The kinks have also been linked to maxima in the spin susceptibility [192]. Of course, additional kinks in the electronic dispersion may also arise from the coupling of electrons to bosonic degrees of freedom, such as phonons or spin fluctuations. Interestingly, recent experiments [183] have found evidence for kinks in $\mathrm{Ni}(110)$, which may be due to the electronic mechanism presented here.

\section{SUMMARY AND OUTLOOK}

Due to the intensive international research over the last two decades the DMFT has quickly developed into a powerful method for the investigation of electronic systems with strong correlations. It provides a comprehensive, non-perturbative and thermodynamically consistent approximation scheme for the investigation of finite-dimensional systems (in particular for dimension $d=3$ ), and is particularly useful for the study of problems where perturbative approaches are inapplicable. For this reason the DMFT has now become the standard mean-field theory for fermionic correlation problems, in- 


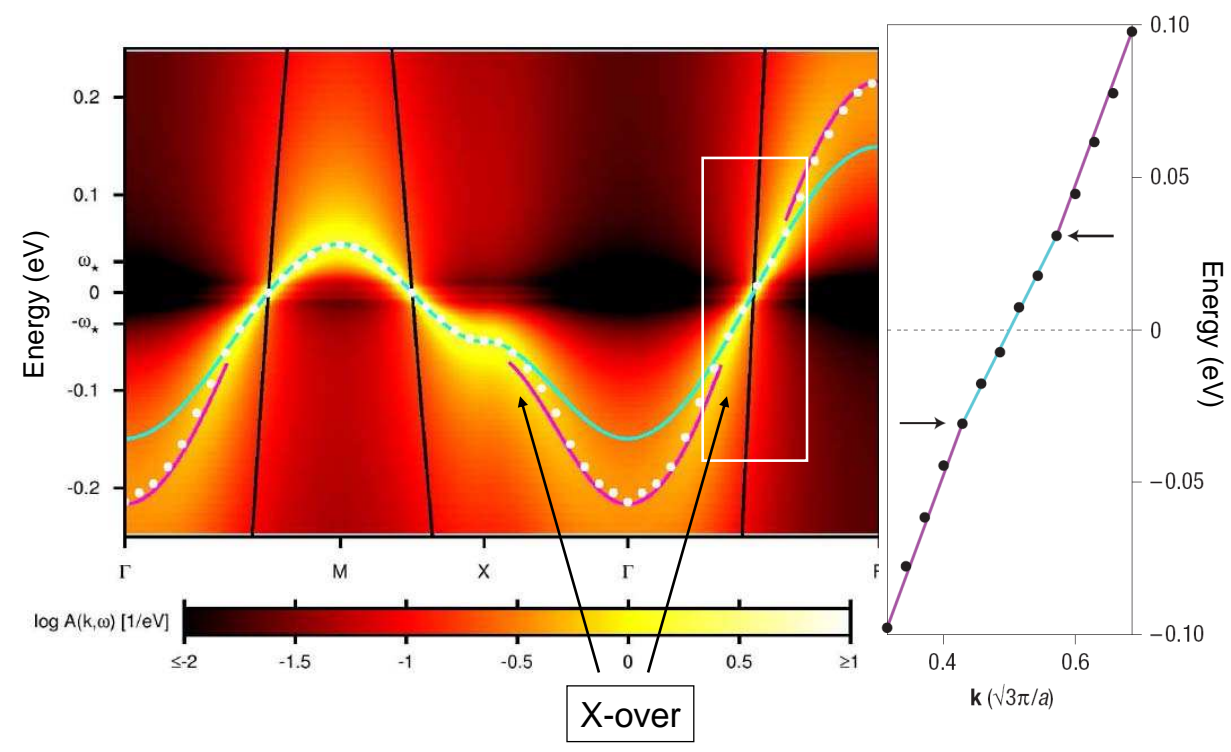

FIGURE 26. Kinks in the dispersion relation $E_{\boldsymbol{k}}$ of the Hubbard model on a cubic lattice with interaction $U=3.5 \mathrm{eV}$, bandwidth $W \approx 3.46 \mathrm{eV}, n=1$, implying a Fermi-liquid renormalization factor $Z_{\mathrm{FL}}=0.086$. The intensity plot represents the spectral function $A(\boldsymbol{k}, \omega)$. Close to the Fermi energy the effective dispersion (white dots) follows the renormalized band structure $E_{k}=Z_{\mathrm{FL}} \varepsilon_{k}$ (light line). For $|\omega|>\omega_{\star}$ the dispersion has the same shape but with a different renormalization, $E_{k}=Z_{\mathrm{CP}} \varepsilon_{k}-c \operatorname{sgn}\left(E_{k}\right)$ (dark line). Here $\omega_{\star}=0.03$ $\mathrm{eV}, Z_{\mathrm{CP}}=0.135$, and $c=0.018 \mathrm{eV}$ are all calculated from $Z_{\mathrm{FL}}$ and $\varepsilon_{k}$ (black line). A subinterval of $\Gamma-\mathrm{R}$ (white frame) is plotted on the right, showing kinks at $\pm \omega_{\star}$ (arrows); after Ref. [188].

cluding cold atoms in optical lattices [184], [120, 193]. The study of models in nonequilibrium within a suitable generalization of the DMFT has become yet another fascinating new research area [194-204].

Until a few years ago research into correlated electron systems concentrated on homogeneous bulk systems. DMFT investigations of systems with internal or external inhomogeneities such as thin films and multi-layered nanostructures are still very new [205-210]. They are particularly important in view of the novel types of functionalities of such systems, which may have important applications in electronic devices. Here the DMFT and its generalizations will certainly be very useful.

In particular, the development of the $a b$ initio band-structure calculation technique referred to as LDA+DMFT has proved to be a breakthrough in the investigation of 
electronically correlated materials. It has already provided important insights into the spectral and magnetic properties of correlated electron materials, e.g., transition metals and their oxides [130, 131], [53, 132-134]. Clearly, this approach has a great potential for further developments. Indeed, it is not hard to foresee that the LDA+DMFT framework will eventually develop into a comprehensive $a b$ initio approach which is able to describe, and even predict, the properties of complex correlated materials.

\section{ACKNOWLEDGMENTS}

These lecture notes gave me the opportunity to discuss concepts and results obtained in collaboration with numerous coauthors over more than 20 years. I wish to express my deep gratitude to all of them, with particular thanks to (in the chronological order of our first coauthorship): Walter Metzner, Florian Gebhard, Peter van Dongen, Vaclav Janiš, Ruud Vlaming, Götz Uhrig, Karsten Held, Marcus Kollar, Nils Blümer, Walter Hofstetter, Thomas Pruschke, Vladimir Anisimov, Ralf Bulla, Igor Nekrasov, Theo Costi, Volker Eyert, Georg Keller, Krzysztof Byczuk, Jim Allen, Shigemasa Suga, Martin Ulmke, Gabi Kotliar, Martin Eckstein, Ivan Leonov, and Jan Kuneš. I am also much obliged to Anna Kauch for her assistance in the preparation of these lecture notes and to Krzysztof Byczuk and Marcus Kollar for helpful discussions. This project was supported in part by the Deutsche Forschungsgemeinschaft through SFB 484 and TRR 80.

\section{REFERENCES}

1. M. Imada, A. Fujimori, and Y. Tokura, Rev. Mod. Phys. 70, 1039 (1998).

2. D. Pines and P. Nozières, The Theory of Quantum Liquids (Benjamin, New York, 1966).

3. G. R. Stewart, Rev. Mod. Phys. 56, 755 (1984).

4. M. C. Gutzwiller, Phys. Rev. Lett. 10, 159 (1963).

5. J. Hubbard, Proc. Roy. Soc. London A276, 238 (1963).

6. J. Kanamori, Prog. Theor. Phys. 30, 275 (1963).

7. $\quad$ E. Lieb and F. Y. Wu, Phys. Rev. Lett. 20, 1445 (1968)

8. R. J. Baxter, Exactly Solved Models in Statistical Mechanics (Academic Press, London, 1982)

9. M. Eckstein, M. Kollar, K. Byczuk and D. Vollhardt, Phys. Rev. B 71, 235119 (2005).

10. C. Itzykson and J.-M. Drouffe, Statistical Field Theory (Cambridge University Press, Cambridge, 1989).

11. D. Vollhardt, in Correlated Electron Systems, edited by V. J. Emery (World Scientific, Singapore, 1993) p. 57.

12. R. Brout, Phys. Rev. 118, 1009 (1960).

13. C. J. Thompson, Commun. Math. Phys. 36, 255 (1974).

14. D. Sherrington and S. Kirkpatrick, Phys. Rev. Lett. 32, 1792 (1975).

15. W. Metzner and D. Vollhardt, Phys. Rev. Lett. 62, 324 (1989).

16. U. Wolff, Nucl. Phys. B 225, 391 (1983).

17. E. Müller-Hartmann, Z. Phys. B 74, 507 (1989).

18. K. Byczuk and D. Vollhardt, Phys. Rev. B 77, 235106 (2008).

19. W. Metzner, Z. Phys. B 77, 253 (1989).

20. E. Müller-Hartmann, Z. Phys. B 76, 211 (1989).

21. U. Brandt and C. Mielsch, Z. Phys. B 75, 365 (1989).

22. H. Schweitzer and G. Czycholl, Solid State Comm. 69, 171 (1989); Z. Phys. B79, 377 (1990); Solid State Commun. 74, 735 (1990); Z. Phys. B83, 93 (1991).

23. P. G. J. van Dongen, F. Gebhard, and D. Vollhardt, Z. Phys. 76, 199 (1989). 
24. F. Kajzar and J. Friedel, J. Phys. (Paris) 39, 397 (1978).

25. G. Treglia, F. Ducastelle, and D. Spanjaard, Phys. Rev. B 22, 6472 (1980).

26. G. Bulk and R. J. Jelitto, Phys. Rev. B 41, 413 (1990).

27. P. G. J. van Dongen, Phys. Rev. Lett. 67, 757 (1991).

28. E. Müller-Hartmann, Int. J. Mod. Phys. B 3, 2169 (1989).

29. J. M. Luttinger and J. C. Ward, Phys. Rev. 118, 1417 (1960).

30. A. C. Hewson, The Kondo Problem to Heavy Fermions (Cambridge University Press, Cambridge, 1997).

31. V. Janiš, Z. Phys. B 83, 227 (1991).

32. V. Janiš and D. Vollhardt, Int. J. Mod. Phys. B 6, 731 (1992).

33. A. Georges and G. Kotliar, Phys. Rev. B 45, 6479 (1992).

34. M. Jarrell, Phys. Rev. Lett. 69, 168 (1992).

35. M. Fabrizio, in Lectures on the Physics of highly correlated electron systems XI, editors A. Avella and F. Mancini, AIP Conference Proceedings, Vol. 918 (American Institute of Physics, Melville, 2007), p. 3.

36. J. E. Hirsch and R. M. Fye, Phys. Rev. Lett. 56, 2521 (1986).

37. D. W. Taylor, Phys. Rev. 156, 1017 (1967).

38. P. Soven, Phys. Rev. 156, 809 (1967).

39. F. Yonezawa and K. Morigaki, Suppl. Prog. Theor. Phys. 53, 1 (1973).

40. R. J. Elliott, J. A. Krumhansl, and P. L. Leath, Rev. Mod. Phys. 46, 465 (1974).

41. P. W. Anderson, Phys. Rev. 109, 1492 (1958).

42. G. Rickayzen, Green's Functions and Condensed Matter (Academic Press, London, 1980).

43. V. Janiš and D. Vollhardt Phys. Rev. B 46, 15712 (1992).

44. E. Müller-Hartmann, Solid State Commun. 12, 1269 (1973).

45. L. Schwartz and E. Siggia, Phys. Rev. B 5, 383 (1972).

46. R. Vlaming and D. Vollhardt, Phys. Rev. B 45, 4637 (1992).

47. V. Janiš, Phys. Rev. B 40, 11331 (1989).

48. J. W. Negele and H. Orland, Quantum Many-Particle Systems (Addison-Wesley, New York, 1988).

49. V. N. Popov, Functional Integrals and Collective Excitations (Cambridge University Press, Cambridge, 1987).

50. V. Janiš, Czech. J. Phys. B 36, 1107 (1986).

51. G. Baym, Phys. Rev. 127, 836 (1962).

52. A. Georges, G. Kotliar, W. Krauth, and M. J. Rozenberg, Rev. Mod. Phys. 68, 13 (1996).

53. G. Kotliar and D. Vollhardt, Physics Today 3, 53 (2004).

54. J. K. Freericks and V. Zlatić, Rev. Mod. Phys. 75, 1333 (2003).

55. X. Y. Zhang, M. J. Rozenberg, and G. Kotliar, Phys. Rev. Lett. 70, 1666 (1993).

56. M. J. Rozenberg, X. Y. Zhang, and G. Kotliar, Phys. Rev. Lett. 69, 1236 (1992).

57. A. Georges and W. Krauth, Phys. Rev. Lett. 69, 1240 (1992).

58. R. Bulla, Phys. Rev. Lett. 83, 136 (1999).

59. R. Bulla, T. A. Costi, and Th. Pruschke, Rev. Mod. Phys. 80, 395 (2008).

60. M. Caffarel and W. Krauth, Phys. Rev. Lett. 72, 1545 (1994).

61. Q. Si, M. J. Rozenberg, G. Kotliar, and A. E. Ruckenstein, Phys. Rev. Lett. 72, 2761 (1994).

62. M. J. Rozenberg, G. Moeller, and G. Kotliar, Mod. Phys. Lett. B 8, 535 (1994).

63. Th. Pruschke, M. Jarrell, and J. K. Freericks, Adv. Phys. 44, 187 (1995).

64. A. Georges, in Lectures on the Physics of highly correlated electron systems VIII, editors A. Avella and F. Mancini, AIP Conference Proceedings, Vol. 715 (American Institute of Physics, Melville, 2004), p. 3.

65. Th. Maier, M. Jarrell, Th. Pruschke, and M. H. Hettler, Rev. Mod. Phys. 77, 1027 (2005).

66. M. Jarrell, A. Macridin, K. Mikelsons, D. G . S. P. Doluweera, and J. E. Gubernatis, in Lectures on the Physics of highly correlated electron systems XII, editors A. Avella and F. Mancini, AIP Conference Proceedings, Vol. 1014 (American Institute of Physics, Melville, 2008).

67. N. F. Mott, Rev. Mod. Phys. 40, 677 (1968).

68. N. F. Mott, Metal-Insulator Transitions (Taylor and Francis, London, 1990), 2 edition

69. F. Gebhard, The Mott Metal-Insulator Transition, Springer, Berlin, 1997.

70. D. B. McWhan and J. P. Remeika, Phys. Rev. B B2, 3734 (1970). 
71. D. B. McWhan, A. Menth, J. P. Remeika, W. F. Brinkman, and T. M. Rice, Phys. Rev. B B2, 1920 (1973).

72. T. M. Rice and D. B. McWhan, IBM J. Res. Develop. 251 (May 1970).

73. J. Hubbard, Proc. Roy. Soc. London A281, 401 (1964).

74. W. F. Brinkman and T. M. Rice, Phys. Rev. B 2, 4302 (1970).

75. M. C. Gutzwiller, Phys. Rev. 137, A1726 (1965).

76. W. Metzner and D. Vollhardt, Phys. Rev. B 37, 7382 (1988).

77. D. Vollhardt, Rev. Mod. Phys. 56, 99 (1984).

78. N. Blümer, Metal-Insulator Transition and Optical Conductivity in High Dimensions (Shaker Verlag, Aachen, 2003).

79. R. Bulla, T. A. Costi, and D. Vollhardt, Phys. Rev. B 64, 045103 (2001).

80. D. Vollhardt, K. Held, G. Keller, R. Bulla, Th. Pruschke, I. A. Nekrasov, and V. I. Anisimov, J. Phys. Soc. Jpn. 74, 136 (2005).

81. S.-K. Mo, H.-D. Kim, J. W. Allen, G. H. Gweon, J. D. Denlinger, J.-H. Park, A. Sekiyama, A. Yamasaki, S. Suga, P. Metcalf, and K. Held, Phys. Rev. Lett. 93, 076404 (2004).

82. M. J. Rozenberg, R. Chitra, and G. Kotliar, Phys. Rev. Lett. 83, 3498 (1999).

83. J. Joo and V. Oudovenko, Phys. Rev. B 64, 193102 (2001).

84. D. Vollhardt and P. Wölfle, The Superfluid Phases of Helium 3 (Taylor and Francis, London, 1990).

85. H. Park, K. Haule, and G. Kotliar, Phys. Rev. Lett. 101, 186403 (2008).

86. Th. Pruschke, Prog. Theor. Phys. Suppl. 160, 274 (2005).

87. N. F. Mott, Proc. Phys. Soc. London, Sect. A 62, 415 (1949).

88. P. A. Lee and T. V. Ramakrishnan, Rev. Mod. Phys. 57, 287 (1985).

89. B.L. Altshuler and A.G. Aronov, in Electron-Electron Interactions in Disordered Systems, eds. M. Pollak and A.L. Efros (North-Holland, Amsterdam, 1985), p.1.

90. D. Belitz and T. R. Kirkpatrick, Rev. Mod. Phys. 66, 261 (1994).

91. E. Abrahams, S.V. Kravchenko, and M.P. Sarachik, Rev. Mod. Phys. 73, 251 (2001).

92. S. V. Kravchenko and M. P. Sarachik, Rep. Prog. Phys. 67, 1 (2004).

93. K. Byczuk, W. Hofstetter, U. Yu, and D. Vollhardt, Eur. Phys. J. Special Topics 180, 135 (2010).

94. K. Byczuk, W. Hofstetter, and D. Vollhardt, Phys. Rev. Lett. 94, 056404 (2005).

95. K. Byczuk, W. Hofstetter, and D. Vollhardt, Phys. Rev. Lett. 102, 146403 (2009).

96. K. Byczuk, W. Hofstetter, and D. Vollhardt, in Fifty Years of Anderson Localization, edited by E. Abrahams (World Scientific, Singapore, 2010), p. 473; reprinted in Int. J. Mod. Phys. B 24, 1727 (2010).

97. A. D. Mirlin and Y. V. Fyodorov, Phys. Rev. Lett. 72, 526 (1994).

98. M. Janssen, Phys. Rep. 295, 1 (1998).

99. A. Richardella, P. Roushan, S. Mack, B. Zhou, D. A. Huse, D. D. Awschalom, and A. Yazdani, Science 327, 665 (2010).

100. G. Schubert, J. Schleede, K. Byczuk, H. Fehske, and D. Vollhardt, Phys. Rev. B 81, 155106 (2010).

101. D. Thouless, Phys. Reports 13, 93 (1974).

102. E. L. Crow and K. Shimizu, editors, Log-normal distribution-theory and applications (Marcel Dekker, Inc., New York, 1988).

103. E. W. Montroll and M. F. Schlesinger, J. Stat. Phys. 32, 209 (1983).

104. M. Romeo, V. D. Costa, and F. Bardou, Eur. Phys. J. B 32, 513 (2003).

105. V. Dobrosavljević and G. Kotliar, Phys. Rev. Lett. 78, 3943 (1997).

106. V. Dobrosavljević, A. A. Pastor, and B. K. Nikolić, Europhys. Lett. 62, 76 (2003).

107. G. Schubert, A. Weisse and H. Fehske, in High Performance Computing in Science and Engineering Garching 2004, edited by A. Bode and F. Durst (Springer-Verlag, Berlin, 2005), p. 237.

108. M. Ulmke, V. Janiš, and D. Vollhardt, Phys. Rev. B 51, 10411 (1995).

109. F. X. Bronold, A. Alvermann, and H. Fehske, Phil. Mag. 84, 637 (2004).

110. M. Lewenstein, A. Sanpera, V. Ahufinger, B. Damski, A. Sen De, and U. Sen, Adv. Phys. 56, 243 (2007).

111. I. Bloch, J. Dalibard, and W. Zwerger, Rev. Mod. Phys. 80, 885 (2008).

112. J. Billy, V. Josse, Z. Zuo, A. Bernard, B. Hambrecht, P. Lugan, D. Clément, L. Sanchez-Palencia, Ph. Bouyer, and A. Aspect, Nature 453891 (2008).

113. G. Roati, Ch. D’Errico, L. Fallani, M. Fattori, Ch. Fort, M. Zaccanti, G. Modugno, M. Modugno, and M. Inguscio, Nature 453, 895 (2008). 
114. A. Aspect and M. Inguscio, Physics Today 62, No. 8 (August), 30 (2009).

115. L. Sanchez-Palencia and M. Lewenstein, Nature Phys. 6, 87 (2010).

116. D. Jaksch, C. Bruder, J.I. Cirac, C.W. Gardiner, and P. Zoller, Phys. Rev. Lett. 81, 3108 (1998).

117. M. Greiner, O. Mandel, T. Esslinger, Th.W. Hänsch, and I. Bloch, Nature 415, 39 (2002).

118. W. Hofstetter, J.I. Cirac, P. Zoller, E. Demler, and M.D. Lukin, Phys. Rev. Lett. 89, 220407 (2002).

119. R. Jördens, N. Strohmaier, K. Günter, H. Moritz, and T. Esslinger, Nature 455, 204 (2008).

120. U. Schneider, L. Hackermüller, S. Will, Th. Best, I. Bloch, T. A. Costi, R. W. Helmes, D. Rasch, A. Rosch, Science 322, 1520 (2008).

121. P. Hohenberg and W. Kohn, Phys. Rev. 136B, 864 (1964).

122. W. Kohn and L. J. Sham, Phys. Rev. 140, A1133 (1965).

123. R. O. Jones and O. Gunnarsson, Rev. Mod. Phys. 61, 689 (1989).

124. V. I. Anisimov, J. Zaanen, and O. K. Andersen, Phys. Rev. B 44, 943 (1991).

125. V. I. Anisimov, F. Aryasetiawan, and A. I. Lichtenstein, J. Phys. Cond. Matter 9, 767 (1997).

126. V. I. Anisimov, A. I. Poteryaev, M. A. Korotin, A. O. Anokhin, and G. Kotliar, J. Phys.: Cond. Matt. 9, 7359 (1997).

127. A. I. Lichtenstein and M. I. Katsnelson, Phys. Rev. B 57, 6884 (1998).

128. I. A. Nekrasov, K. Held, N. Blümer, A. I. Poteryaev, V. I. Anisimov, and D. Vollhardt, Eur. Phys. J. $B$ 18, 55 (2000).

129. K. Held, I. A. Nekrasov, N. Blümer, V. I. Anisimov, and D. Vollhardt, Int. J. Mod. Phys. B 15, 2611 (2001).

130. A. I. Lichtenstein, M. I. Katsnelson, and G. Kotliar, in Electron Correlations and Materials Properties, edited by A. Gonis, N. Kioussis, and M. Ciftan (Kluwer Academic/Plenum, New York, 2002), p. 428.

131. K. Held, I. A. Nekrasov, G. Keller, V. Eyert, N. Blümer, A. K. McMahan, R. T. Scalettar, T. Pruschke, V. I. Anisimov, and D. Vollhardt, Psi-k Newsletter 56, 65 (2003); reprinted in Phys. Status Solidi B 243, 2599 (2006).

132. G. Kotliar, S. Y. Savrasov, K. Haule, V. S. Oudovenko, O. Parcollet, C. A. Marianetti, Rev. Mod. Phys. 78, 865 (2006)

133. K. Held, Adv. Phys. 56, 829 (2007)

134. M. I. Katsnelson, V. Yu. Irkhin, L. Chioncel, A. I. Lichtenstein, and R. A. de Groot, Rev. Mod. Phys. 80,315 (2008)

135. J. Kuneš, I. Leonov, M. Kollar, K. Byczuk, V. I. Anisimov, and D. Vollhardt, Eur. Phys. J. Special Topics 180, 5 (2010).

136. M. Jarrell and J. E. Gubernatis, Phys. Rep. 269, 133 (1996).

137. A. Fujimori, I. Hase, H. Namatame, Y. Fujishima, Y. Tokura, H. Eisaki, S. Uchida, K. Takegahara, and F. M. F. de Groot, Phys. Rev. Lett. 69, 1796 (1992).

138. A. Sekiyama, H. Fujiwara, S. Imada, S. Suga, H. Eisaki, S. I. Uchida, K. Takegahara, H. Harima, Y. Saitoh, I. A. Nekrasov, G. Keller, D. E. Kondakov, A. V. Kozhevnikov, T. Pruschke, K. Held, D. Vollhardt, and V. I. Anisimov, Phys. Rev. Lett. 93, 156402 (2004).

139. I. A. Nekrasov, G. Keller, D. E. Kondakov, A. V. Kozhevnikov, Th. Pruschke, K. Held, D. Vollhardt, and V. I. Anisimov, Phys. Rev. B 72, 155106 (2005).

140. E. Pavarini, S. Biermann, A. Poteryaev, A. I. Lichtenstein, A. Georges, and O. K. Andersen, Phys. Rev. Lett. 92, 176403 (2004).

141. I. H. Inoue, I. Hase, Y. Aiura, A. Fujimori, K. Morikawa, T. Mizokawa, Y. Haruyama, T. Maruyama, and Y. Nishihara, Physica C 235-240, 1007 (1994).

142. M. O. Krause and J. H. Oliver, J. Phys. Chem Ref. Data 8, 329 (1979).

143. I. H. Inoue (2003), private communication.

144. I. H. Inoue, C. Bergemann, I. Hase, and S. R. Julian, Phys. Rev. Lett. 88, 236403 (2002).

145. J. H. de Boer and E. J. W. Verwey, Proc. Phys. Soc. 49, No. 4S, 59 (1937).

146. N. F. Mott, Proc. Phys. Soc. A 49, 57 (1937).

147. J. Kuneš, V. I. Anisimov, A. V. Lukoyanov, D. Vollhardt, Phys. Rev. B 75, 165115 (2007).

148. J. Zaanen, G. A. Sawatzky, and J. W. Allen, Phys. Rev. Lett. 55, 418 (1985).

149. V. Emery, Phys. Rev. Lett. 58, 2794 (1987).

150. R. E. Cohen, I. I. Mazin, and D. G. Isaak, Science 275, 654 (1997).

151. C. S. Yoo, B. Maddox, J.-H. P. Klepeis, V. Iota, W. Evans, A. McMahan, M. Y. Hu, P. Chow, M. Somayazulu, D. Häusermann, W. E. Pickett, and R. T. Scalettar, Phys. Rev. Lett. 94, 115502 
(2005).

152. L. F. Mattheiss, Phys. Rev. B 5, 290 (1972).

153. K. Terakura, A. R. Williams, T. Oguchi, and J. Kübler, Phys. Rev. B 30, 4734 (1984).

154. A. Fujimori, F. Minami, and S. Sugano, Phys. Rev. B 29, 5225 (1984).

155. V. I. Anisimov, J. Zaanen, and O. K. Andersen, Phys. Rev. B 44, 943 (1991).

156. A. Svane and O. Gunnarsson, Phys. Rev. Lett. 65, 1148 (1991).

157. F. Arysetiawan and O. Gunnarsson, Phys. Rev. Lett. 74, 3221 (1995).

158. S. Y. Savrasov and G. Kotliar, Phys. Rev. Lett. 90, 056401 (2003).

159. G. A. Sawatzky and J. W. Allen, Phys. Rev. Lett. 53, 2339 (1984).

160. D. E. Eastman and J. L. Freeouf, Phys. Rev. Lett. 34, 395 (1975).

161. J. Kuneš, V. I. Anisimov, S. L. Skornyakov, A. V. Lukoyanov, D. Vollhardt, Phys. Rev. Lett. 99, $156404(2007)$

162. K. I. Kugel and D. I. Khomskii, Sov. Phys. Usp. 25, 231 (1982).

163. O. K. Andersen, Phys. Rev. B 12, 3060 (1975).

164. I. Leonov, N. Binggeli, D. Korotin, V. I. Anisimov, N. Stojić, and D. Vollhardt, Phys. Rev. Lett. 101, 096405 (2008).

165. I. Leonov, Dm. Korotin, N. Binggeli, V. I. Anisimov, D. Vollhardt, Phys. Rev. B 81, 075109 (2010).

166. A. K. McMahan, K. Held, and R. T. Scalettar, Phys. Rev. B 67, 075108 (2003).

167. B. Amadon, S. Biermann, A. Georges, and F. Aryasetiawan, Phys. Rev. Lett. 96, 066402 (2006).

168. S. Y. Savrasov, G. Kotliar, and E. Abrahams, Nature 410, 793 (2001).

169. X. Dai, S. Y. Savrasov, G. Kotliar, A. Migliori, H. Ledbetter, and E. Abrahams, Science 300, 953 (2003)

170. S. Y. Savrasov and G. Kotliar, Phys. Rev. B 69, 245101 (2004).

171. J. Kuneš, A. V. Lukoyanov, V. I. Anisimov, R. T. Scalettar, and W. E. Pickett, Nature Materials 7, 198 (2008).

172. G. Trimarchi, I. Leonov, N. Binggeli, D. Korotin, and V. I. Anisimov, J. Phys.: Cond. Matt. 20, 135227 (2008).

173. F. Lechermann, A. Georges, A. Poteryaev, S. Biermann, M. Posternak, A. Yamasaki, and O. K. Andersen, Phys. Rev. B 74, 125120 (2006).

174. A. I. Liechtenstein, V. I. Anisimov, and J. Zaanen, Phys. Rev. B 52, 5467 (1995).

175. J. E. Medvedeva, M. A. Korotin, V. I. Anisimov, and A. J. Freeman, Phys. Rev. B 65, 172413 (2002).

176. R. H. Buttner, E. N. Maslen, and N. Spadaccini, Acta Cryst. B 46, 131 (1990).

177. A. Lanzara, P. V. Bogdanov, X. J. Zhou, S. A. Kellar, D. L. Feng, E. D. Lu, T. Yoshida, H. Eisaki, A. Fujimori, K. Kishio, J.-I. Shimoyama, T. Noda, S. Uchida, Z. Hussain, and Z.-X. Shen, Nature 412, $510(2001)$.

178. Z.-X. Shen, A. Lanzara, S. Ishihara, and N. Nagaosa, Philos. Mag. B 82, 1349 (2002).

179. H. He, Y. Sidis, P. Bourges, G. D. Gu, A. Ivanov, N. Koshizuka, B. Liang, C. T. Lin, L. P. Regnault, E. Schoenherr, and B. Keimer, Phys. Rev. Lett. 86, 1610 (2001).

180. J. Hwang, T. Timusk, and G. D. Gu, Nature 427, 714 (2004).

181. M. Hengsberger, D. Purdie, P. Segovia, M. Garnier, and Y. Baer, Phys. Rev. Lett. 83, 592 (1999).

182. E. Rotenberg, J. Schaefer, and S. D. Kevan, Phys. Rev. Lett. 84, 2925 (2000).

183. A. Hofmann, X. Y. Cui, J. Schäfer, S. Meyer, P. Höpfner, C. Blumenstein, M. Paul, L. Patthey, E. Rotenberg, J. Bünemann, F. Gebhard, T. Ohm, W. Weber, R. Claessen, Phys. Rev. Lett. 102, 187204 (2009).

184. A. Rapp, G. Zarand, C. Honerkamp, W. Hofstetter, Phys. Rev. Lett. 98, 160405 (2007).

185. J. Schäfer, D. Schrupp, E. Rotenberg, K. Rossnagel, H. Koh, P. Blaha, and R. Claessen, Phys. Rev. Lett. 92, 097205 (2004)

186. Y. Aiura, Y. Yoshida, I. Hase, S. I. Ikeda, M. Higashiguchi, X. Y. Cui, K. Shimada, H. Namatame, M. Taniguchi, and H. Bando, Phys. Rev. Lett. 93, 117005 (2004).

187. T. Yoshida, K. Tanaka, H. Yagi, A. Ino, H. Eisaki, A. Fujimori, and Z.-X. Shen, Phys. Rev. Lett. 95, 146404 (2005).

188. K. Byczuk, M. Kollar, K. Held, Y.-F. Yang, I. A. Nekrasov, T. Pruschke, and D. Vollhardt, Nature Phys. 3, 168 (2007).

189. K. Byczuk, W. Hofstetter, M. Kollar, and D. Vollhardt, Acta Physica Polonica A 111, 549 (2007).

190. I. A. Nekrasov, K. Held, G. Keller, , D. E. Kondakov, Th. Pruschke, M. Kollar, O. K. Andersen, V. I. Anisimov, and D. Vollhardt, Phys. Rev. B 73, 155112 (2006). 
191. A. Toschi, M. Capone, C. Castellani, K. Held, Phys. Rev. Lett. 102, 076402 (2009).

192. C. Raas, P. Grete, G. S. Uhrig, Phys. Rev. Lett. 102, 076406 (2009).

193. M. Snoek, I. Titvinidze, C. Toke, K. Byczuk, and W. Hofstetter, New J. Phys. 10, 093008 (2008).

194. P. Schmidt and H. Monien, arXiv:cond-mat/0202046.

195. V. Turkowski and J. K. Freericks, Phys. Rev. B 71, 085104 (2005).

196. J. K. Freericks, V. M. Turkowski, and V. Zlatić, Phys. Rev. Lett. 97266408 (2006).

197. M.-T. Tran, Phys. Rev. B 78, 125103 (2008).

198. N. Tsuji, T. Oka, and H. Aoki, Phys. Rev. B 78, 235124 (2008).

199. J. K. Freericks, Phys. Rev. B 77, 075109 (2008).

200. J. K. Freericks, H. R. Krishnamurthy, and Th. Pruschke, Phys. Rev. Lett. 102, 136401 (2009).

201. M. Eckstein, and M. Kollar, Phys. Rev. Lett. 100, 120404 (2008).

202. M. Eckstein, and M. Kollar, Phys. Rev. B 78, 205119 (2008).

203. M. Eckstein, and M. Kollar, Phys. Rev. B 78, 245113 (2008).

204. M. Eckstein, M. Kollar, and P. Werner, Phys. Rev. Lett. 103, 056403 (2009).

205. M. Potthoff and W. Nolting, Phys. Rev. B 59, 2549 (1999).

206. J. K. Freericks, Transport in multilayered nanostructures - The dynamical mean-field approach (Imperial College Press, London, 2006).

207. M. Takizawa, H. Wadati, K. Tanaka, M. Hashimoto, T. Yoshida, A. Fujimori, A. Chikamtsu, H. Kumigashira, M. Oshima, K. Shibuya, T. Mihara, T. Ohnishi, M. Lippmaa, M. Kawasaki, H. Koinuma, S. Okamoto, and A. J. Millis, Phys. Rev. Lett. 97, 057601 (2006).

208. L. Chen and J. K. Freericks, Phys. Rev. B 75, 1251141 (2007).

209. K. Byczuk, in Condensed Matter Physics in the Prime of the 21st Century: Phenomena, Materials, Ideas, Methods, ed. J. Jedrzejewski (World Scientific, Singapore, 2008), p. 1.

210. R. W. Helmes, T. A. Costi, and A. Rosch, Phys. Rev. Lett. 100, 056403 (2008). 